\title{
ACCurate Measurement of THE X-RAy CoHEREnT SCATTERING FORM FACTORS OF TISSUES
}

by

Brian W. King

A Thesis Submitted to the Faculty of Graduate Studies and Research in Partial Fulfillment of the Requirements for the degree of

Doctor of Philosophy

Ottawa Carleton Institute for Physics

Department of Physics

Carleton University

Ottawa, Canada

September 2009

(c)Brian W. King, September 2009. All rights reserved. 
Library and Archives Canada

Published Heritage Branch

395 Wellington Street Ottawa ON K1A ON4 Canada
Bibliothèque et

Archives Canada

Direction du

Patrimoine de l'édition

395, rue Wellington

Ottawa ON K1A ON4

Canada
Your file Votre reference

ISBN: 978-0-494-60112-9

Our file Notre reférence

ISBN: 978-0-494-60112-9

\section{NOTICE:}

The author has granted a nonexclusive license allowing Library and Archives Canada to reproduce, publish, archive, preserve, conserve, communicate to the public by telecommunication or on the Internet, loan, distribute and sell theses worldwide, for commercial or noncommercial purposes, in microform, paper, electronic and/or any other formats.

The author retains copyright ownership and moral rights in this thesis. Neither the thesis nor substantial extracts from it may be printed or otherwise reproduced without the author's permission.
AVIS:

L'auteur a accordé une licence non exclusive permettant à la Bibliothèque et Archives Canada de reproduire, publier, archiver, sauvegarder, conserver, transmettre au public par télécommunication ou par l'Internet, prêter, distribuer et vendre des thèses partout dans le monde, à des fins commerciales ou autres, sur support microforme, papier, électronique et/ou autres formats.

L'auteur conserve la propriété du droit d'auteur et des droits moraux qui protège cette thèse. $\mathrm{Ni}$ la thèse ni des extraits substantiels de celle-ci ne doivent être imprimés ou autrement reproduits sans son autorisation.
In compliance with the Canadian Privacy Act some supporting forms may have been removed from this thesis.

While these forms may be included in the document page count, their removal does not represent any loss of content from the thesis.
Conformément à la loi canadienne sur la protection de la vie privée, quelques formulaires secondaires ont été enlevés de cette thèse.

Bien que ces formulaires aient inclus dans la pagination, il n'y aura aucun contenu manquant.

\section{Canadä}




\section{ABSTRACT}

The material dependent $\mathrm{x}$-ray scattering properties of tissues are determined by their scattering form factors, measured as a function of the momentum transfer argument, $x$. Incoherent scattering form factors, $F_{\mathrm{inc}}$, are calculable for all values of $x$ while coherent scattering form factors, $F_{\text {coh }}$, cannot be calculated except at large $x$ because of their dependence on long range order. As a result, measuring $F_{\mathrm{coh}}$ is very important to the developing field of $\mathrm{x}$-ray scatter imaging.

Previous measurements of $F_{\mathrm{coh}}$, based on crystallographic techniques, have shown significant variability, as these methods are not optimal for amorphous materials. Two methods of measuring $F_{\text {coh }}$, designed with amorphous materials in mind, are developed in this thesis.

An angle-dispersive technique is developed that uses a polychromatic x-ray beam and a large area, energy-insensitive detector. It is shown that $F_{\text {coh }}$ can be measured in this system if the incident $\mathrm{x}$-ray spectrum is known. The problem is ill-conditioned for typical $\mathrm{x}$-ray spectra and two numerical methods of dealing with the poor conditioning are explored. It is shown that these techniques work best with K-edge filters to limit the spectral width and that the accuracy degrades for strongly ordered materials. Measurements of $F_{\text {coh }}$ for water samples are made using 50,70 and $92 \mathrm{kVp}$ spectra. The average absolute relative difference in $F_{\text {coh }}$ between our results and the literature for water is approximately $10-15 \%$. Similar measurements for fat samples were made and found to be qualitatively similar to results in the literature, although there is very large variation between the literature values in this case. The angle-dispersive measurement is limited to low resolution 
measurements of the coherent scattering form factor although it is more accessible than traditional measurements because of the relatively commonplace equipment requirements.

An energy-dispersive technique is also developed that uses a polychromatic $\mathrm{x}$-ray beam and an energy-sensitive detector. It is shown that $F_{\text {coh }}$ can be measured directly by computing the ratio of two spectra: one, measured at a given scattering angle and the other, the direct transmission spectrum with no scattering. Experiments were constructed on this principle and used to measure $F_{\text {coh }}$ for water, fat and several types of plastics. A $121 \mathrm{kVp}$ x-ray spectrum and seven different scattering angles between $1.75^{\circ}$ and $15^{\circ}$ were used, resulting in a measurable range of $x$ between 0.5 and $9.5 \mathrm{~nm}^{-1}$. These are the first measurements of coherent scattering form factors made without the need for a scaling factor.

Resolution in $x$ varies between $10 \%$ at small scattering angles and $2 \%$ for larger scattering angles. Accuracy in $F_{\text {coh }}$ is shown to be strongly dependent on the precision of the experimental geometry and varies between $5 \%$ and $15 \%$. The average absolute relative difference between repeated measurements was $\sim 5 \%$.

The agreement between the energy-dispersive and angle-dispersive techniques is shown to be quite good, considering the low resolution of the angle-dispersive measurement. The average absolute relative difference in $F_{\text {coh }}$ between the angle-dispersive measurement with a $70 \mathrm{kVp}$ spectrum and the energy-dispersive measurement was $\sim 12 \%$. 


\section{ACKNOWLEDGEMENTS}

I would like to thank my supervisor, Paul Johns, for his help and suggestions throughout this project. I would also like to thank Phillippe Gravelle in the Physics Department machine shop for his assistance with equipment fabrication. There were a number of students working in the $\mathrm{x}$-ray lab that contributed to this work. Charles Steiner, Susan Oda, Kevin Earl and Karl Landheer all helped make this possible. Dr. Ken Storey of the Carleton University Biochemistry Institute graciously allowed access to his storage plate reader for

the image plate measurements in Chapter 4. Dr. David Rogers provided a dual channel electrometer for the fluence ratio measurements in Chapter 5. 
For Heather, Lyta and Bryce 


\section{LIST OF ABBREVIATIONS}

$\begin{array}{ll}\mathrm{ADC} & \text { Analog-To-Digital Converter } \\ \mathrm{Am} & \text { Americium } \\ \mathrm{BaFBr} & \text { Barium Fluorobromide } \\ \mathrm{Be} & \text { Beryllium } \\ \mathrm{BGO} & \text { Bismuth Germanate }-\mathrm{Bi}_{4} \mathrm{Ge}_{3} \mathrm{O}_{12} \\ \mathrm{CdZnTe} & \text { Cadmium Zinc Telluride } \\ \mathrm{CR} & \text { Computed Radiography } \\ \mathrm{CT} & \text { Computed Tomography } \\ \mathrm{HPGe} & \text { High Purity Germanium } \\ \mathrm{IAM} & \text { Independent Atom Model } \\ \mathrm{keV} & \text { kilo electron-Volts } \\ \mathrm{kVp} & \text { kilo-Voltage peak } \\ \mathrm{MCA} & \text { Multi-Channel Analyzer } \\ \mathrm{MeV} & \text { Mega electron-Volts } \\ \mathrm{Mo} & \text { Molybdenum } \\ \mathrm{NaI} & \text { Sodium Iodide } \\ \mathrm{RMS} & \text { Root Mean Square } \\ \mathrm{Pb} & \text { Lead } \\ \mathrm{PBS} & \text { Phosphate Buffered Saline } \\ \mathrm{W} & \text { Tungsten }\end{array}$




\section{CONTENTS}

Abstract $\quad$ ii

Acknowledgements $\quad$ iv

$\begin{array}{ll}\text { List of Abbreviations } & \text { vi }\end{array}$

Table of Contents $\quad$ vii

$\begin{array}{ll}\text { List of Figures } & \mathbf{x}\end{array}$

List of Tables $\quad$ xiv

1 Introduction $\quad 1$

1.1 Conventional X-Ray Imaging . . . . . . . . . . . . . . . . 1

1.2 Scatter X-Ray Imaging . . . . . . . . . . . . . . . . 4

1.3 Coherent Scattering Form Factor Measurements . . . . . . . . . . 7

1.3.1 Diffractometer Measurements ............. 8

1.3.2 Alternative Measurements ............... 11

1.4 Outline of the Thesis ........................ 12

2 Theory 14

2.1 Production of X Rays . . . . . . . . . . . . . . . . 14

2.2 X-Ray Interactions in Matter . . . . . . . . . . . . . . 17

2.2.1 Coherent Scattering . . . . . . . . . . . . 17

2.2.2 Incoherent Scattering . . . . . . . . . . . . . 21 
2.2 .3 Total Scattering . . . . . . . . . . . . . . . . . . 24

2.3 Detector Technology $\ldots \ldots \ldots \ldots \ldots \ldots \ldots$

2.3 .1 Storage Plates . . . . . . . . . . . . . . 25

2.3.2 Ionization Chambers . . . . . . . . . . . . . . . 27

2.3.3 Germanium Spectrometers . . . . . . . . . . . . . 27

3 Angle-Dispersive Form Factor Measurements using Imaging Detectors Development $\quad 34$

3.1 Model Development . . . . . . . . . . . . . . . . . . . . . . . . . 34

3.2 Mathematical Methods . . . . . . . . . . . . . . . 38

3.2.1 Effective Energy Approximation . . . . . . . . . . . 38

3.2.2 Sub-matrix Calculation . . . . . . . . . . . 38

3.2.3 Regularization Calculation $\ldots \ldots \ldots \ldots \ldots$. . . . . 41

3.3 Simulation Results $\ldots \ldots \ldots \ldots \ldots$. . . . . . . . . 42

3.3.1 Sub-Matrix Calculations $\ldots \ldots \ldots \ldots \ldots \ldots \ldots$

3.3.2 Regularization Calculations . . . . . . . . . . . . . . 48

3.4 Discussion . . . . . . . . . . . . . . . . 50

4 Angle-Dispersive Form Factor Measurements using Imaging Detectors Results

4.1 Experimental Details $\ldots \ldots \ldots \ldots \ldots \ldots \ldots$

4.2 Measuring $\mathrm{d} P / \mathrm{d} E \ldots \ldots \ldots \ldots \ldots \ldots \ldots$

4.3 Form Factor Measurements . . . . . . . . . . . . . . . . 60

4.4 Discussion . . . . . . . . . . . . . . . . . 63

5 Energy-Dispersive Form Factor Measurements - Development 66

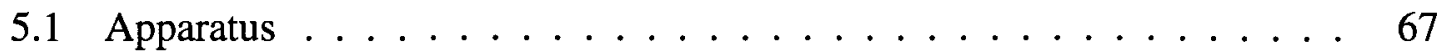

$5.1 .1 \quad$ Apertures $\ldots \ldots \ldots \ldots \ldots \ldots \ldots \ldots$

5.1 .2 X-ray tube $\ldots \ldots \ldots \ldots \ldots \ldots \ldots \ldots \ldots \ldots$ 
5.1 .3 HPGe Detector $\ldots \ldots \ldots \ldots \ldots \ldots \ldots \ldots$

5.1 .4 Target Station $\ldots \ldots \ldots \ldots \ldots \ldots \ldots \ldots \ldots \ldots \ldots$

5.1 .5 Spectral Measurements . . . . . . . . . . . . . 72

5.2 Theory . . . . . . . . . . . . . . . . . 74

$5.2 .1 \quad$ Parameter calculation $\ldots \ldots \ldots \ldots \ldots \ldots$

5.2 .2 Overall Fluence ratio $\ldots \ldots \ldots \ldots \ldots \ldots$

6 Energy-Dispersive Form Factor Measurements - Results 81

6.1 Results . . . . . . . . . . . . . . . . . . . 81

6.1 .1 Water Measurements ................ 84

6.1 .2 Plastic Measurements . . . . . . . . . . . . . . . . . 88

6.1 .3 Fat measurements . . . . . . . . . . . . 88

6.1 .4 Combining individual measurements . . . . . . . . . . . . . 89

6.2 Discussion . . . . . . . . . . . . . . . . 93

$\begin{array}{llr}7 & \text { Conclusions } & 98\end{array}$

7.1 Discussion . . . . . . . . . . . . . . . . . 100

7.2 Future Work . . . . . . . . . . . . . . . . . . . . . 103

7.3 Final Thoughts . . . . . . . . . . . . . . . . . . 104

A Form Factor Results $\quad 106$

B Energy Dispersive Alignment Procedure 113

$\begin{array}{ll}\text { C Source Code Directory } & 118\end{array}$

C.1 Top-Level Programs . . . . . . . . . . . . . . . . . . 118

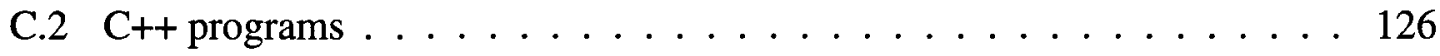

C.3 C++ Libraries . . . . . . . . . . . . . . . . . . . . 127

$\begin{array}{lr}\text { References } & 129\end{array}$ 


\section{LIST OF FIGURES}

1.1 Schematic representation of conventional x-ray imaging process $\ldots \ldots 2$

1.2 Sample projection $x$-ray image $\ldots \ldots \ldots \ldots \ldots$

1.3 Comparison of interaction cross-sections for water . . . . . . . . 5

1.4 Contours of constant $x \ldots \ldots \ldots \ldots \ldots \ldots$

1.5 Schematic representation of an $\mathrm{x}$-ray diffractometer . . . . . . . 9

1.6 Coherent scattering form factors of water . . . . . . . . . . 10

1.7 Coherent scattering form factors of tissues $\ldots \ldots \ldots \ldots \ldots$

2.1 Methods of $x$-ray production. . . . . . . . . . . . . 15

2.2 Example x-ray spectrum $\ldots \ldots \ldots \ldots \ldots \ldots$

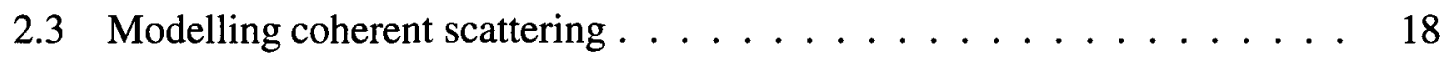

2.4 Contours of the $F_{\mathrm{KN}}$ function for diagnostic energies. . . . . . . 22

2.5 Calculated values of $F_{\text {inc }} \ldots \ldots \ldots \ldots \ldots \ldots \ldots \ldots \ldots \ldots$

2.6 Coherent scatter fraction for water at different scattering angles calculated using the independent atom model. . . . . . . . . . . . . . . 24

2.7 The image acquisition process in phosphor screens $\ldots \ldots \ldots 26$

2.8 Schematic representation of HPGe spectrometer operation. . . . . . . 28

2.9 X-ray spectra of monochromatic source measured with an ideal spectrometer and a real spectrometer . . . . . . . . . . . . . . . 29

$2.10 \mathrm{X}$-ray interaction processes occurring within a HPGe detector. . . . . . 30

2.11 Contribution of different interactions to detector response function. . . . . 32 
2.12 Effect of detector correction on measured tungsten anode $\mathrm{x}$-ray spectrum .

3.1 Ideal experimental layout modelled for angle-dispersive form factor mea-

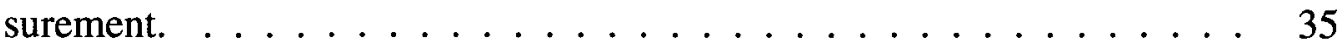

3.2 Diagrammatic representation of sub-matrix construction. . . . . . . 40

3.3 Example sub-matrix calculations for water and fat. . . . . . . . . 43

3.4 Accuracy of the sub-matrix calculation for a range of spectral shapes. . . 44

3.5 Accuracy of the sub-matrix calculation for a range of form factors. . . . . 45

3.6 Sub-matrix calculation for water showing the need for sub-matrix truncation. 46

3.7 Sub-matrix calculation for water showing the effect of spectrum truncation. 47

3.8 Comparison of the regularization calculation with varying smoothness parameters. . . . . . . . . . . . . . . . . . . . . 49

3.9 Accuracy of the regularization calculation with a range of spectral shapes. 49

3.10 Accuracy of the regularization calculation with a range of artificial form

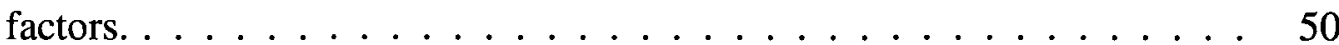

4.1 Experimental layout for form factor measurement with imaging detector. . 53

4.2 Scatter patterns of water measured using different spectra. . . . . . . . 55

4.3 Repeatability of measured scatter patterns. . . . . . . . . . . . 56

4.4 Average scatter signal of water and fat measured with imaging detector. . $\quad 57$

4.5 Spectra used in angle-dispersive measurements. . . . . . . . . . . . 58

4.6 Calculated $\mathrm{d} P / \mathrm{d} E$ function for $50 \mathrm{kVp}$ beam and a water target. . . . . 59

4.7 Form factors for water extracted from experimental angle-dispersive mea-

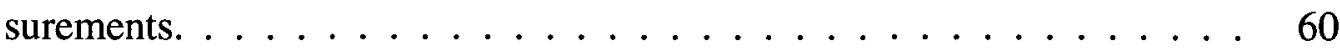

4.8 Form factors for fat extracted from experimental angle-dispersive mea-

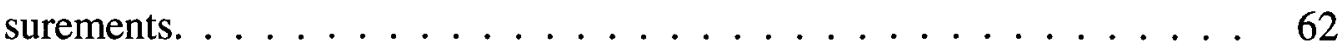

5.1 Schematic of experimental setup for energy-dispersive measurement. . . . 66

5.2 Detailed experimental setup for energy-dispersive form factor measurements 67 
5.3 Typical energy dependence of effective transmission aperture area. . . . . 71

5.4 Photo of the energy-dispersive target station. . . . . . . . . . 73

6.1 Measured transmission and scatter spectra for water $\ldots \ldots \ldots$. . . . . 82

6.2 Form factor measurements for water from each scattering configuration with an energy-dispersive measurement. . . . . . . . . . .

6.3 Uncertainties in experimental parameters for the energy-dispersive measurement. . . . . . . . . . . . . . . . . . . . . . . . .

6.4 Uncertainty estimates of momentum transfer argument for the energydispersive method for individual scattering apertures. . . . . . . . 86

6.5 Uncertainty estimates of $F_{\text {coh }}$ for the energy-dispersive method for individual scattering apertures. . . . . . . . . . . . . 87

6.6 Measured form factors of water with three different target thicknesses. . . 87

6.7 Form factor measurements for polyethylene with the energy-dispersive method. . . . . . . . . . . . . . . . . . . 88

6.8 Form factor measurements for fat with the energy-dispersive method. . . . 89

6.9 Combined form factor measurements of water with the energy-dispersive method.

6.10 Combined form factor measurements of polyethylene with the energydispersive method. . . . . . . . . . . . . . . . . . . . . . 91

6.11 Combined form factor measurements of fat with the energy-dispersive method.

6.12 Combined form factor measurements of plastics with the energy-dispersive method.

6.13 Form factor of water measured using the HPGe detector correction and

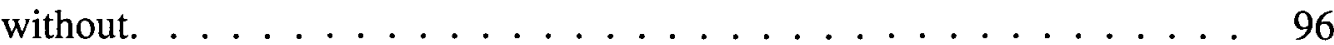


7.1 Comparison of energy-dispersive and sub-matrix form factor measure-

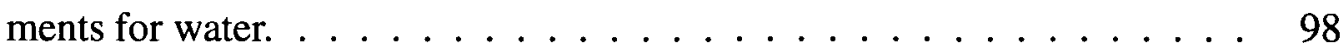

7.2 Comparison of energy-dispersive and sub-matrix form factor measurements for fat. . . . . . . . . . . . . . . . 99

7.3 Sketch of ideal and actual apertures. . . . . . . . . . . . 102

B.1 Equipment used in energy-dispersive form factor measurement. . . . . . . 114

B.2 Bench mounted equipment used in energy-dispersive form factor measurement. . . . . . . . . . . . . . . . . . 114 


\section{LIST OF TABLES}

1.1 Properties of several common materials in the human body. . . . . . . . 3

2.1 Characteristic radiation energies of materials. . . . . . . . 16

2.2 Monochromatic sources used to measure detector response function of the HPGe detector. . . . . . . . . . . . . . . . . 32

3.1 Parameters used in constructing artificial form factors . . . . . . . . 45

4.1 Collimation dimensions for angle-dispersive form factor measurement with

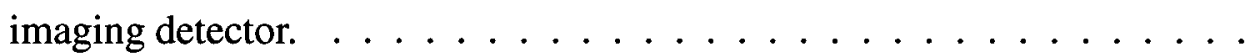

4.2 Exposure parameters used for angle-dispersive form factor measurements with imaging detector. . . . . . . . . . . . . . . . . 54

4.3 Spectrum truncation used with each input $d \Phi / d E$ spectrum for the sub-

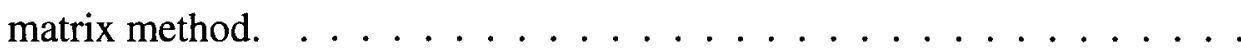

4.4 Average absolute relative difference for angle-dispersive form factor measurements of water as compared to published data. . . . . . . . . .

4.5 Average absolute relative difference for angle-dispersive form factor measurements of fat as compared to published data. . . . . . . . . .

5.1 Object locations for energy-dispersive form factor measurements. . . . . .

5.2 Available aperture dimensions used for the energy-dispersive form factor

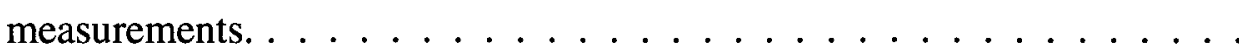


5.3 Aperture configurations used for energy-dispersive form factor measurements. . . . . . . . . . . . . . . . . . 69

5.4 Scattering parameters calculated from Monte Carlo simulation. . . . . . . 78

5.5 Measured fluence ratios for scattering apertures. . . . . . . . . . . 80

6.1 Compositions and parameters of water, fat, and plastics. . . . . . . . 83

6.2 Average absolute relative difference of the combined form factor measurements compared to published values. . . . . . . . . . . . 93

7.1 Average absolute relative difference between angle and energy-dispersive measurements. . . . . . . . . . . . . . . . . . . . . 99

A.1 Measured form factor of water using the energy-dispersive technique. . . 107

A.2 Measured form factor of fat using the energy-dispersive technique. . . . . 108

A.3 Measured form factor of polyethylene using the energy-dispersive technique.109

A.4 Measured form factor of polycarbonate using the energy-dispersive tech-

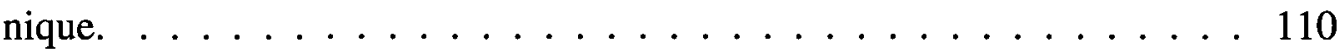

A.5 Measured form factor of polystyrene using the energy-dispersive technique. 111

A.6 Measured form factor of nylon using the energy-dispersive technique. . . 112 


\section{CHAPTER 1}

\section{INTRODUCTION}

$\mathrm{X}$ rays have been a fundamental tool in medical imaging for over a century since their discovery in 1895 by Wilhelm Roentgen. Many significant improvements have been made in x-ray imaging over the years, including the development of anti-scatter grids, dualenergy imaging, computer assisted tomography (CT) and computed radiography (CR) detectors. All of these developments have focused on the same underlying property of $x$ rays: their ability to make "shadow" images based on their attenuation in different types of matter. From this point of view, $\mathrm{x}$ rays are simply point particles.

$\mathrm{X}$ rays, however, exhibit wave properties like other forms of electromagnetic radiation. They can be scattered or diffracted when interacting with matter. The effects of this scattering behaviour are usually difficult to observe because of the very small wavelength of the radiation. There is a great deal of information potentially available from the wave properties of $\mathrm{x}$ rays, as has been known for many years in the field of crystallography where $\mathrm{x}$-ray diffraction has become a standard technique.

In this thesis, I will develop methods for measuring some of the important x-ray scattering properties of biological tissues with the aim of providing a library of scattering data to aid in the development of $x$-ray scatter imaging.

\subsection{Conventional X-Ray Imaging}

Conventional x-ray imaging is based on the differences in the attenuation coefficient between various materials. Dense, high atomic number objects such as bones cause a larger 
number of photons to be removed from the beam than lighter, low atomic number materials such as tissue or air. This principle allows projection images to be produced with an $\mathrm{x}$-ray source and detector as shown schematically in Figure 1.1.

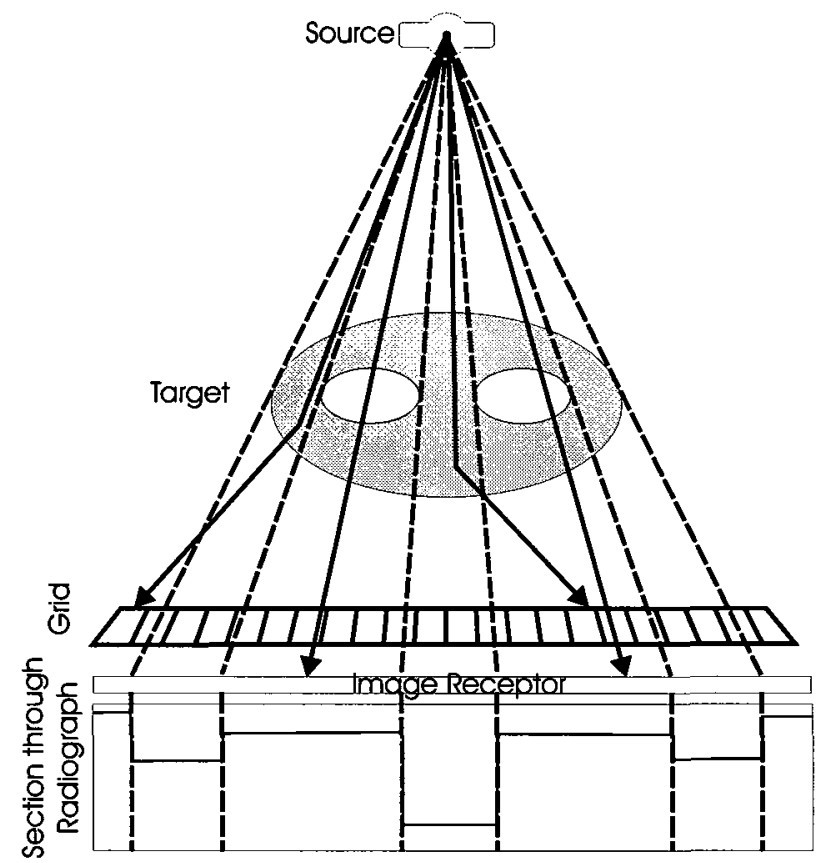

Figure 1.1: Schematic representation of conventional x-ray imaging process (not drawn to scale).

For $\mathrm{x}$-ray energies typically used in medical imaging $(15-150 \mathrm{keV})$, the attenuation coefficient of a material is determined primarily by two factors: atomic number and density. The attenuation coefficient increases as either the atomic number or density are increased. As can be seen in Table 1.1, this means that $\mathrm{x}$ rays are very good at distinguishing between things like bones, tissues and air but are not good at distinguishing between different types of tissues. This is borne out by Figure 1.2 where the air-filled lungs are quite clearly seen but the different types of tissues cannot be distinguished from each other. 
Table 1.1: Properties of several common materials in the human body. Data taken from Appendix A-3 of Reference [1].

\begin{tabular}{lll}
\hline Material & Density $\left(\mathrm{g} / \mathrm{cm}^{3}\right)$ & Average Atomic Number \\
\hline \hline Air & 0.0012 & 7.78 \\
Water & 1.00 & 7.51 \\
Fat & 0.916 & 6.46 \\
Muscle & 1.040 & 7.64 \\
Bone & 1.650 & 12.31 \\
\hline
\end{tabular}

Figure 1.2: Sample projection x-ray image, from the collection of Paul Johns.

A number of techniques have been developed to improve tissue contrast in x-ray images. Materials with large atomic numbers can be used as contrast agents (iodine in the bloodstream and barium in the gastro-intestinal tract are commonly used). Images measured with different $\mathrm{x}$-ray energies can be combined together to highlight the tissues in the body and minimize the effect of bone[2]. Computerized displays that allow adjustable 
contrast allow smaller differences in attenuation to be observed. Multiple projection images can be taken from different orientations and used to generate a full, three-dimensional image. Because the resulting image is no longer a two dimensional projection, small differences in attenuation are easier to see[3]. While these techniques have all made huge improvements in image quality, they are fundamentally limited by the dependence of the signal on the attenuation coefficient.

\subsection{Scatter X-Ray Imaging}

Scattered $\mathrm{x}$ rays were observed to be a problem from the earliest days of conventional $\mathrm{x}$ ray imaging. $\mathrm{X}$ rays scattered in the body no longer followed the straight line path required for projection imaging causing a reduction in contrast and signal-to-noise ratio. This effect was reduced through the development of anti-scatter grids. It is also possible, however, to make use of the way $\mathrm{x}$ rays scatter in different tissues to provide additional information about the tissue.

There are two types of x-ray scattering that can occur in tissues. Incoherent scattering occurs when high energy photons interact with electrons through the Compton effect, while coherent scattering is based on $\mathrm{x}$-ray diffraction. Overall, incoherent scattering is a more significant interaction mechanism than coherent scattering as illustrated in Figure 1.3. In spite of this, coherent scattering can give rise to more significant improvements in image quality because the diffraction process is dependent on structural factors of the tissues and not just on the attenuation coefficient, and due to the fact that it is much more strongly forward directed[4]. This makes collecting the coherently scattered $\mathrm{x}$ rays much more efficient.

A number of groups have attempted to use both incoherent and coherently scattered $\mathrm{x}$ rays in the field of medical physics. The first person to develop an imaging system using incoherently scattered radiation was Lale[6]. His system used Ir-192 photons and a 


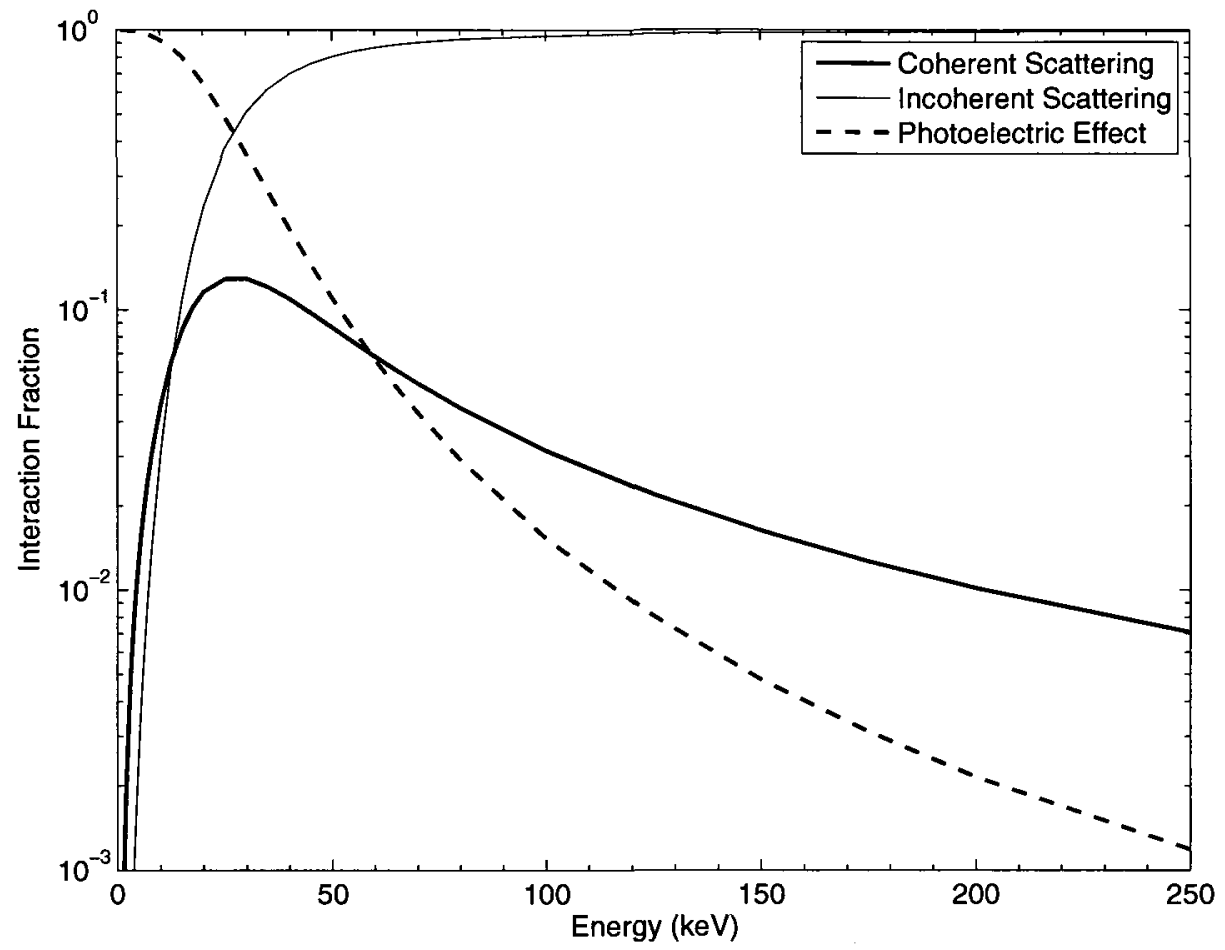

Figure 1.3: Comparison of overall interaction probabilities for water. Calculated from tables produced from [5].

perspex detector crystal with photo-multiplier tube. The process was slow (approximately 10 hours to scan a 3 in. $x 3$ in. area) and produced unclear images.

Clarke and Van Dyk developed a system for measuring bone mineral content using a sodium iodide detector that used incoherent scattering[7]. This method used two high energy radioactive sources to compare scattered and transmitted radiation intensity along different paths. This information can be used to determine the electron density of a small section of the target. The system was designed to measure the properties of a small section of the body as opposed to generating overall images. Scaling the system up to generate large scale images would be straightforward but very time consuming due to the requirement for multiple scans at different orientations.

Farmer and Collins developed an actual imaging system based on incoherent scattering that used an energy sensitive germanium detector and a monochromatic collimated Cs-137 source to measure cross-sectional slices through a target $[8,9]$. This system was similar 
in scope to early computerized tomography systems but was fundamentally limited due to the requirement for a monochromatic pencil beam of photons. Computerized tomography soon became the dominant source for three dimensional $\mathrm{x}$-ray images.

Brateman, Jacobs and Fitzgerald developed a system, called SCAT-CAT[10], to create a tomographic image from the backscatter (incoherent) signal in addition to a standard transmission CT image. They used a single NaI detector at a backscattering angle of $30^{\circ}$ and reconstructed scatter images of several plastic phantoms. The system was sensitive to noise but showed some increased contrast of sub-surface features as compared to the transmission CT images.

Harding's group developed a similar tomographic system designed to image the forward (coherent) scattered $\mathrm{x}$ rays[11]. They used a series of BGO scintillators arranged in a rough series of annular rings about the primary in order to more efficiently collect the scattered photons. Images were made of a phantom with five different types of plastic inserts. The scatter images collected at different scattering angles showed very different amounts of contrast between the different inserts, highlighting one of the advantages of imaging with coherent scatter.

Harding's group also developed an energy-dispersive system to image the coherently scattered $\mathrm{x}$ rays[12]. This system used a HPGe spectrometer to make energy-resolved measurements of the coherently-scattered $\mathrm{x}$-ray photons from a series of plastic targets. A series of annular apertures were used to define a fixed scattering angle from a single small section of the target. This allowed for a localized measurement of the coherent scattering. In principle then, this system allowed three-dimensional images of the coherent scattering to be obtained for an inhomogeneous object.

Leclair and Johns developed a model[13] to quantify the improvement in contrast that is possible by measuring scattered $\mathrm{x}$ rays in various configurations and also validated this model with experiments $[4,14]$. They considered a series of possible imaging options that included collecting only the small angle scattering, only the backwards scattering, and 
also collecting the scattering over all angles. They found that substantial improvements in contrast and signal-to-noise ratio over transmission imaging are possible, depending on the imaging task.

Ian Cunningham's group has used scatter imaging to determine the composition of bone[15] and kidney stones[16]. They developed a system that measured the small angle coherent scattering of a small object in a series of projections, analogous to what is done in computed tomography. They used a tungsten anode $\mathrm{x}$-ray tube, filtered with gadolinium to reduce the spectral width, and an image intensifier system to collect the coherent scatter patterns. The scatter patterns were used to reconstruct a three dimensional representation of the coherent scattering from the object. They have shown that these images can provide relevant physiological information that is currently not clinically available.

\subsection{Coherent Scattering Form Factor Measurements}

As pointed out by Leclair and Johns[4], the scattering properties of tissues can make a critical difference to the signal quality in $\mathrm{x}$-ray scatter imaging. The property that determines a material's x-ray scattering behaviour is the form factor. Form factors are measured as a function of the momentum transfer argument, typically referred to as $x$ :

$$
x=\frac{1}{\lambda} \sin \left(\frac{\theta}{2}\right)=\frac{E}{h c} \sin \left(\frac{\theta}{2}\right),
$$

where $\lambda$ and $E$ are the wavelength and energy, respectively, of the incoming $\mathrm{x}$ ray and $\theta$ is the scattering angle made by the photon. In this equation, $h$ and $c$ are respectively Planck's constant and the speed of light. It will be shown in Chapter 2 that $x$ represents a reciprocal length in the Fourier space of the electron distribution. Thus, small $x$ corresponds to large distances while large $x$ corresponds to short distances. Form factors for both coherent and incoherent scattering can be defined and calculated for individual atoms based on quantum mechanical models of the elements. These form factors have been tab- 
ulated previously[17]. In the case of incoherent scattering, these calculated Independent Atom Model (IAM) form factors provide an accurate measure of a material's scattering properties. In the case of coherent scattering however, the calculated atomic form factors are not accurate for bulk materials [18]. This is because of the diffraction nature of coherent scattering and its dependence on long range order. Coherent scattering occurs as a result of constructive interference of $\mathrm{x}$ rays scattering from many different atoms. As a result, coherent scattering form factors cannot be calculated by current means and must be measured. Values of $x$ above $6 \sim 10 \mathrm{~nm}^{-1}$ correspond to scattering within a single atom or molecule. In this region, known as the IAM region, the coherent scattering form factor can be reliably calculated from atomic form factors. Unfortunately, there is no clear boundary for the IAM region.

To make use of the coherently scattered $\mathrm{x}$ rays in the field of medical physics, form factors of tissues and phantom materials must be known for the range of energies and scattering angles available. Diagnostic energies range from around $15 \mathrm{keV}$ for mammography to as high as $150 \mathrm{keV}$ for chest $\mathrm{x}$ rays or $\mathrm{CT}$ scans and scatter could be collected over virtually any angular range from $1^{\circ}$ to $180^{\circ}$. Figure 1.4 then shows that form factors must be known for a range of $x$ values from $0.1 \mathrm{~nm}^{-1}$ well into the IAM region above $x \sim 10 \mathrm{~nm}^{-1}$.

\subsubsection{Diffractometer Measurements}

The typical method of measuring coherent scattering form factors is based on crystallography instrumentation. A diffractometer is constructed by placing a relatively low-energy source of $\mathrm{x}$ rays and a detector on separate, movable arms about a common centre. Cobalt and copper anode $\mathrm{x}$-ray tubes are commonly used as the source of $\mathrm{x}$ rays. The detector is equipped with a monochromator to filter out photons of unwanted energies. The source and detector can be rotated around the centre to measure the scattering at a range of angles. This is illustrated schematically in Figure 1.5. 


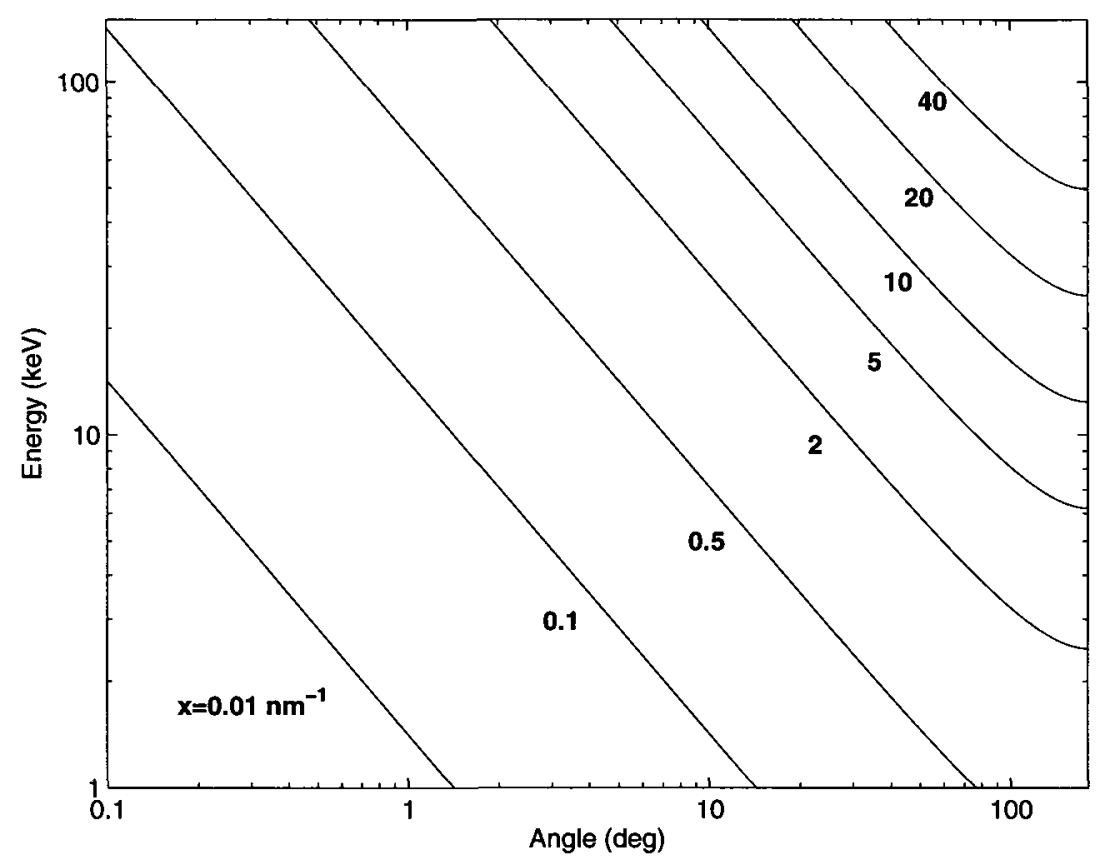

Figure 1.4: Contours of constant $x$ as defined by Equation 1.1.

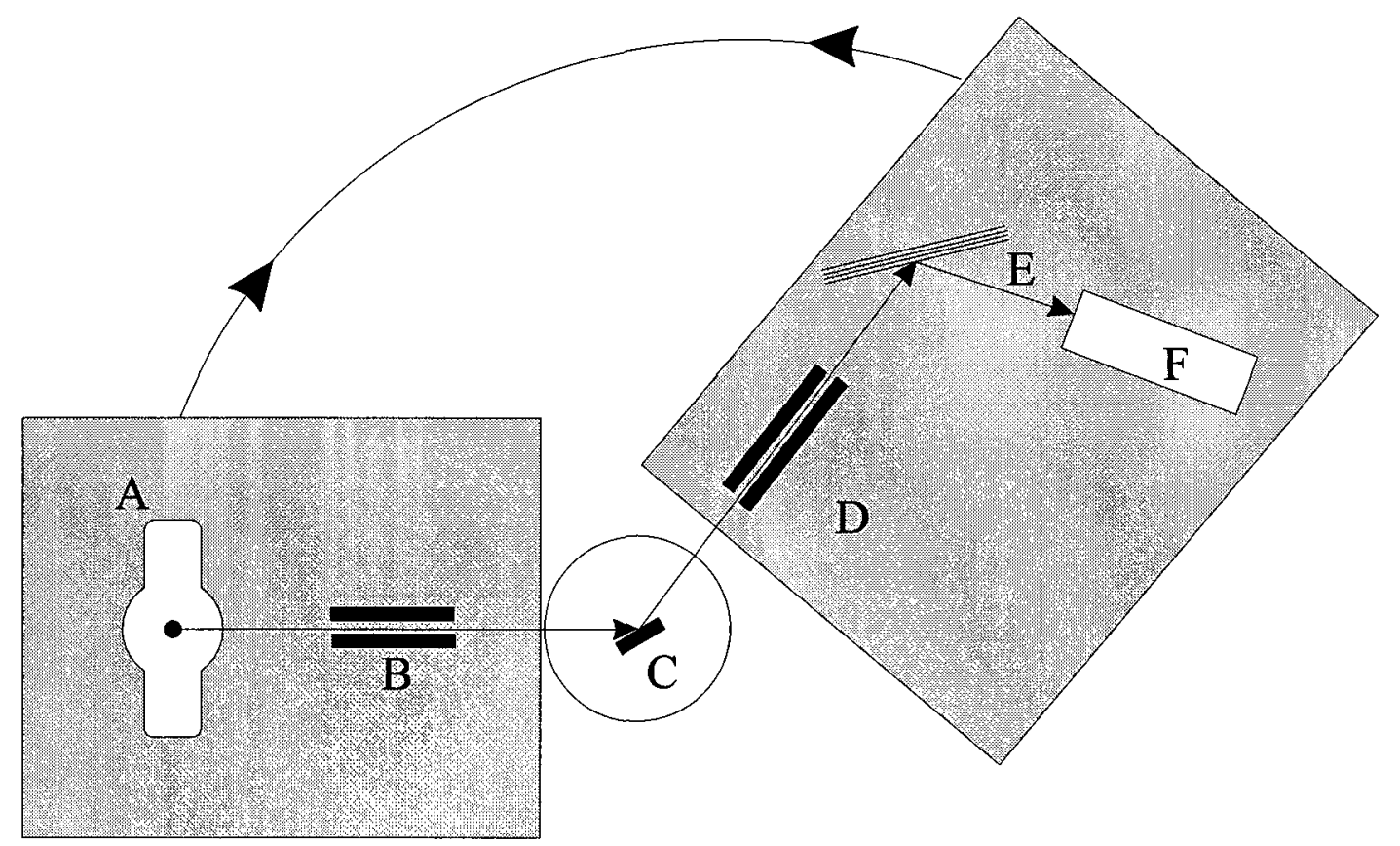

Figure 1.5: Schematic representation of an $x$-ray diffractometer. The components labelled on the sketch are: A - X-ray source, B - Source collimation, C- Target, D - Detector collimation, E - Monochromator, F - Detector. 
The problems with previous diffractometer based measurements of coherent scattering form factors are two-fold: First, not very many materials have been studied in any detail. Water has been studied extensively by Narten using a molybdenum x-ray tube[19]. Others have measured x-ray scattering from water using copper anode x-ray tubes[20] and synchrotron sources[21, 22]. General purpose tissue measurements, on the other hand have only been made by two groups $[20,21]$.

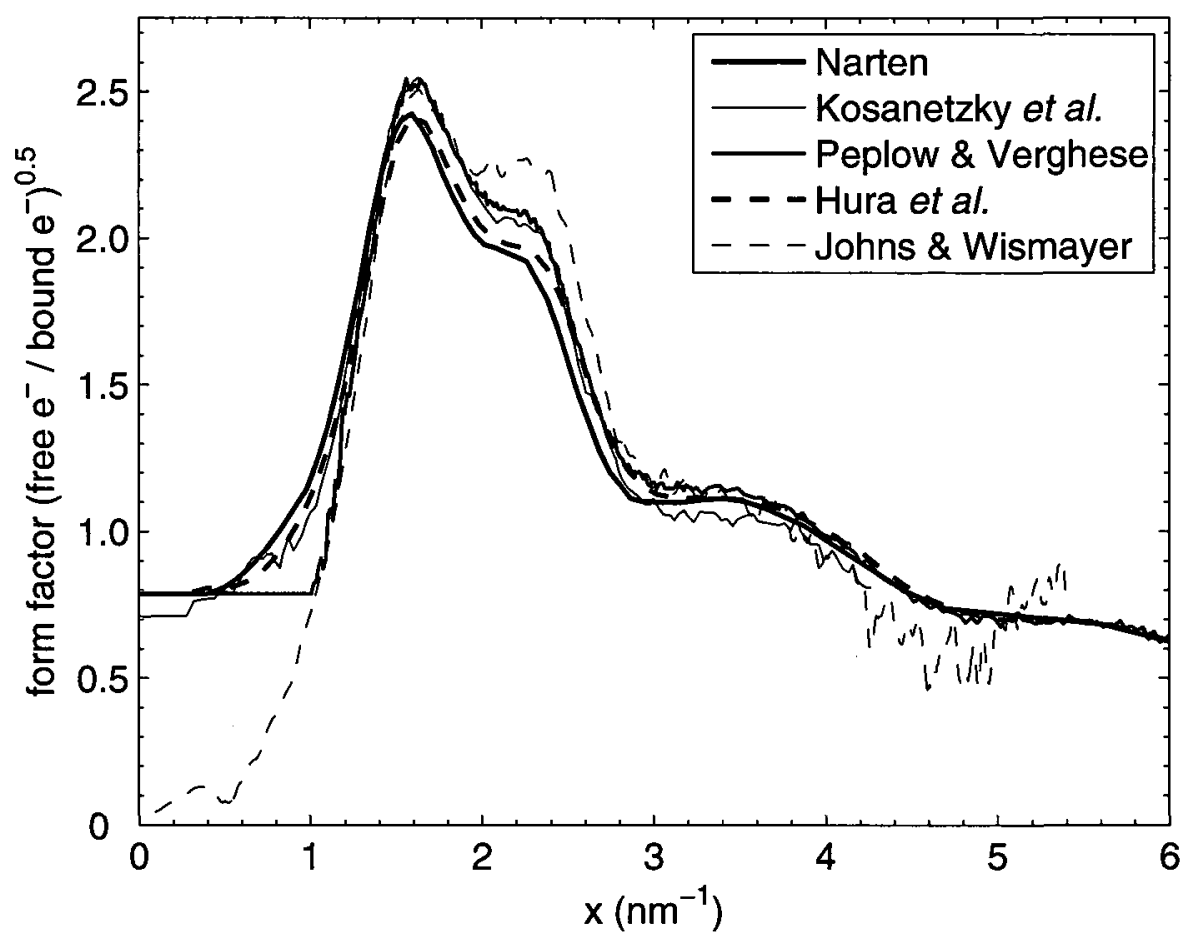

Figure 1.6: Coherent scattering form factors for water extracted from several different studies[19, 20,21, 22, 23]. The Johns and Wismayer data shown here are from the Rigaku diffractometer of Reference [23]. For a discussion of the units for $F_{\text {coh }}$, see Section 2.2.1.

Secondly, there are potentially severe systematic errors that can be present when applying crystallography based diffraction techniques to amorphous materials such as tissues [23]. Amorphous materials do not have the well-ordered crystal planes that give strong diffraction signals in crystallography. Factors such as the polarization efficiency of the monochromator, primary contamination effects and imperfect background subtraction can be important in this case. It is unclear how significant these effects were in previous 
studies, raising additional questions about the accuracy of these results. To illustrate the problems, form factor measurements of water and some tissues by different groups are shown in Figures 1.6 and 1.7. Although the different measurements show the same general trends, significant variations exist between the different measurements.
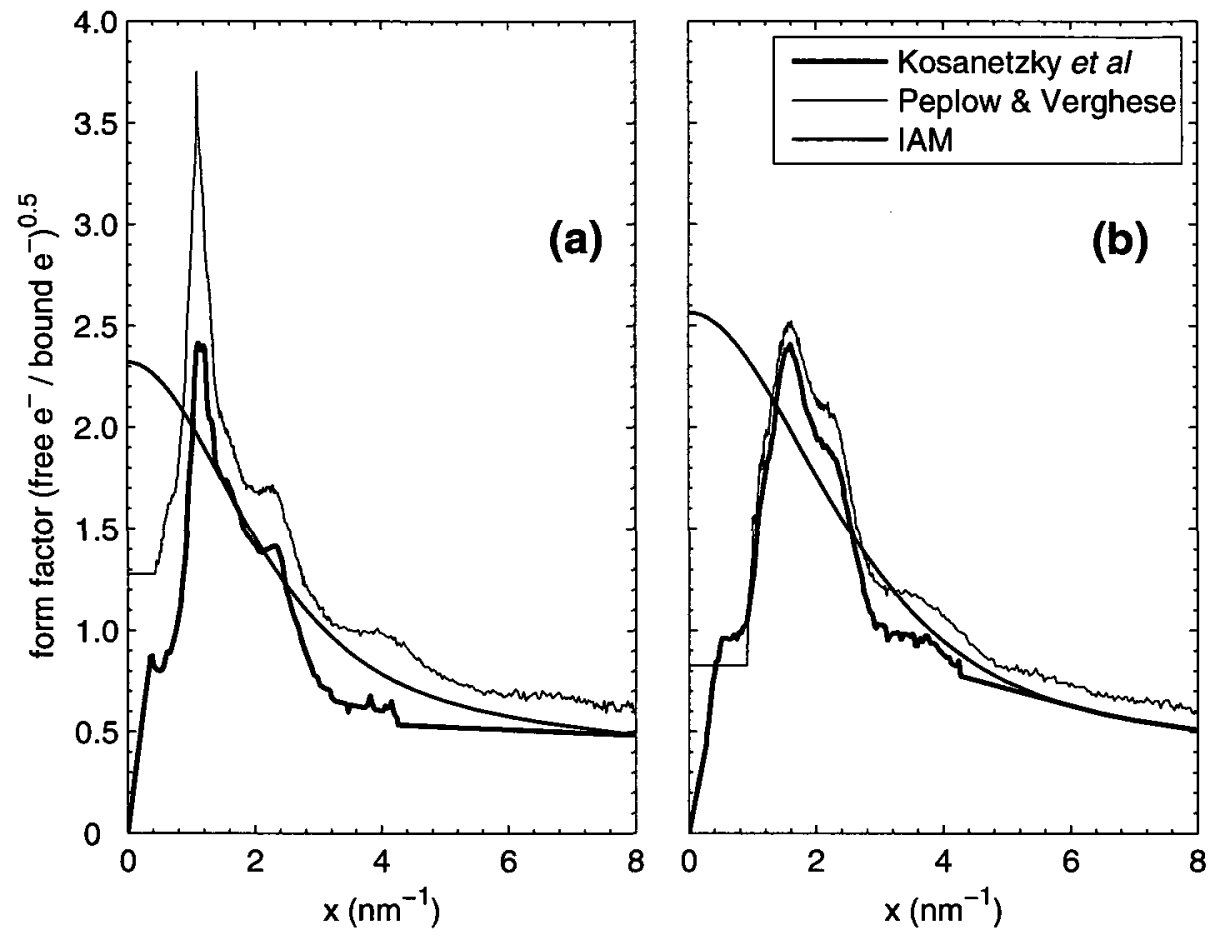

Figure 1.7: Coherent scattering form factors for (a) fat and (b) muscle extracted from different studies[20, 21]. For a discussion of the units for $F_{\mathrm{coh}}$, see Section 2.2.1.

As a result of these problems, we have been investigating alternative methods of measuring coherent scattering form factors.

\subsubsection{Alternative Measurements}

I have developed an alternative method of measuring coherent scattering form factors based on a polychromatic source and an energy insensitive detector, such as a standard $\mathrm{x}$-ray image screen or image intensifier. In this method, the form factor is extracted from 
scatter measurements based on knowledge of the $\mathrm{x}$-ray source and detector properties. This method will be discussed in detail in Chapters 3 and 4 .

I have also developed an energy-dispersive method of measuring coherent scattering form factors using an energy-sensitive detector and a polychromatic source. In this case, the scattering angle is kept fixed and the form factor is extracted from the scattered x-ray spectrum. Previous measurements made by our group have been encouraging but suffered from noise issues due to the small number of photons measured[24].

Similar work has been done by Leclair's group [25] with a focus on the application to breast cancer detection. Their work measures the differential linear scattering coefficient which is related to the coherent scattering form factor. They measured scattering of water and different types of breast tissue using a W anode $\mathrm{x}$-ray tube and CdZnTe spectrometer.

\subsection{Outline of the Thesis}

In this work, I develop several methods to measure the coherent scattering form factor of tissues using standard laboratory sources of $\mathrm{x}$ rays. In chapter 2 , I show how form factors can be used to explain a material's scattering properties. I also describe the basic theory behind the production and measurement of $\mathrm{x}$ rays. In this chapter, $\mathrm{I}$ also explain how spectra measured with a high purity germanium (HPGe) spectrometer can be corrected for the spectrometer's non-ideal behaviour. I developed and tested an algorithm to perform this correction. Implementation and experimental verification were performed by Charles Steiner as a part of his Phys 4908 research project under my day-to-day supervision.

In chapter 3, I explore the possibility of measuring form factors using polychromatic sources and energy-insensitive detectors. I show that the problem is ill-conditioned for realistic x-ray spectra and develop two numerical methods that allow low resolution measurements of the coherent scattering form factor of tissues to be made if the $\mathrm{x}$-ray spectrum and detector properties are known. The results are verified experimentally in Chapter 4 . 
Much of these chapters has been previously published [26] and I have presented the work at conferences[27, 28].

In chapters 5 and 6, I develop an energy-dispersive technique to measure coherent scattering form factors using an energy-sensitive high purity germanium (HPGe) spectrometer. This technique is a highly optimized version of that produced by Ziaul Hasan during his Master's thesis[24, 29]. I developed a sophisticated Monte Carlo ray tracing simulation to model the experimental geometry and to quantitatively assess the level of uncertainty in the measurements. Preliminary results of this work were presented at two conferences $[30,31]$. Technical work done when I had to specify the purchase of, and install a new detector using mechanical cooling rather than liquid nitrogen was also presented[32].

The form factors of water and several plastics were measured using the energy-dispersive technique. The laboratory work was done under my day-to-day supervision by Karl Landheer, who joined our lab under the Natural Sciences and Engineering Research Council Undergraduate Student Research Award program. The results were compared to previous measurements available in the literature. I show that the energy-dispersive technique that I developed produces superior results compared to angle-dispersive measurements. Measurements of the form factor of fat were also made in order to begin developing a library of tissue scattering properties for the development of the field of $\mathrm{x}$-ray scatter imaging. 


\section{CHAPTER 2}

\section{THEORY}

$\mathrm{X}$ rays are a form of high energy electromagnetic radiation produced when electrons are accelerated or undergo transition between atomic energy levels. Typical $\mathrm{x}$ ray energies range from $1 \mathrm{keV}$ up to many hundred $\mathrm{keV}$. Because of their high energy, they can penetrate large distances through typical materials. This makes them a very useful tool in a wide range of fields. Also because of their high energy, they can cause electrons to be ejected from atoms as they pass through. This ionization is responsible for the health issues related to the use of $\mathrm{x}$ rays in living things. In this chapter, I develop the theory necessary to understand the production, interaction and measurement of $\mathrm{x}$ rays.

\subsection{Production of X Rays}

In classical electrodynamics, electromagnetic radiation is produced when charged particles are accelerated. In order to create high energy $\mathrm{x}$-ray photons, these charged particles (typically electrons) need to undergo very large accelerations. $\mathrm{X}$ rays can be produced in several ways, including the use of $\mathrm{x}$-ray tubes and linear accelerators. Both of these methods require the electrons to be moving at very high speeds. In the case of an x-ray tube, electrons are accelerated using a simple high voltage anode and cathode. Using this technology, electrons can be accelerated up to several hundred keV. The high speed electrons then hit a metal target where they produce a great deal of heat as well as $\mathrm{x}$ rays as they decelerate. 
Electrons travelling through a metal target produce $\mathrm{x}$ rays through two separate processes as illustrated in Figure 2.1. Bremsstrahlung radiation is produced when the electron decelerates due to interactions with the target. The energy of the bremsstrahlung photon depends on how sharply the electron changes direction. Since the interaction details are random, the bremsstrahlung process gives rise to a spectrum of $\mathrm{x}$-ray energies. When the high speed electron ionizes one of the atoms in the target, the vacancy in the electronic structure can be filled by another electron falling into the vacant energy level. This transition causes a characteristic $\mathrm{x}$ ray to be produced. Because the energy levels of the atom are quantized, the characteristic radiation has only a few well-defined energies. These energies are dependent on the target material only. Characteristic radiation energies for several common x-ray targets are given in Table 2.1.
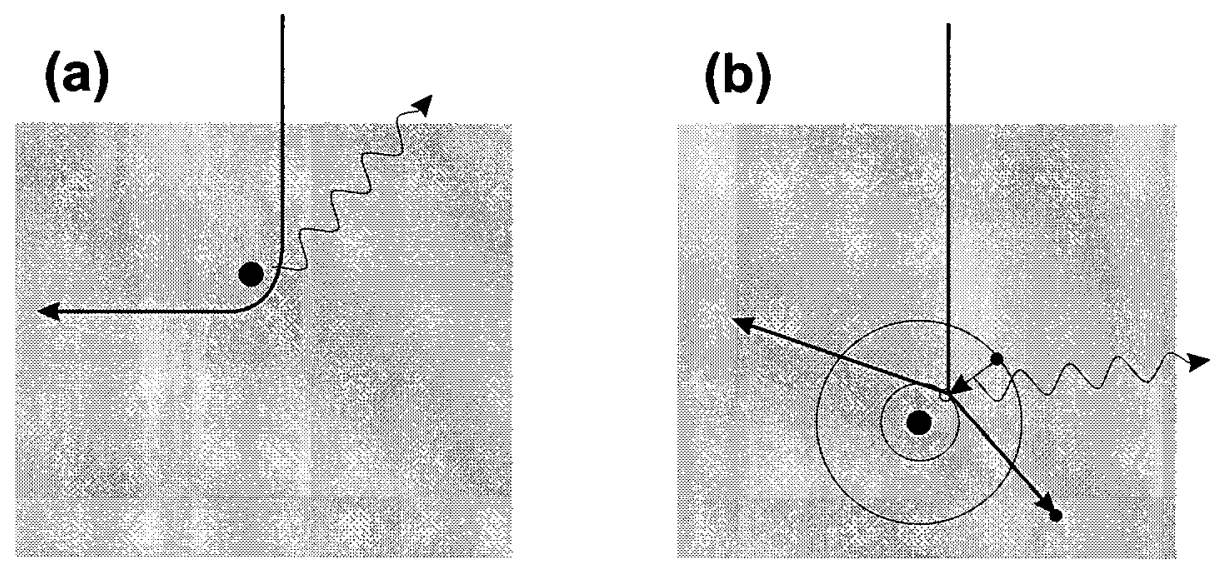

Figure 2.1: Methods of $x$ ray production. Panel (a) shows Bremsstrahlung production and (b) shows production of characteristic radiation in a target.

Linear accelerators function on the same principle as x-ray tubes except that they use a series of accelerating waveguides to achieve much higher electron energies. Electron, and hence photon, energies as high as $20 \mathrm{MeV}$ are commonly available for radiation therapy in cancer centres around the world.

$\mathrm{X}$-ray tubes are the most commonly available method of $\mathrm{x}$-ray production. Typical $\mathrm{x}$ ray tubes used for medical imaging use a tungsten target and give $\mathrm{x}$-ray photons between 
Table 2.1: Characteristic radiation energies of materials. (Data taken from Appendix 3 of [33].)

\begin{tabular}{lcccc}
\hline \multirow{2}{*}{ Material } & \multicolumn{4}{c}{ Characteristic Energies (keV) } \\
Transition Line & \multicolumn{1}{c}{$\alpha_{2}$} & \multicolumn{1}{c}{$\mathrm{K} \alpha_{1}$} & \multicolumn{1}{c}{$\mathrm{K} \beta_{1}$} & $\mathrm{~K} \beta_{2}$ \\
\hline \hline Copper & 8.027 & 8.048 & 8.91 & \\
Molybdenum & 17.374 & 17.479 & 19.60 & 19.97 \\
Tungsten & 57.982 & 59.318 & 67.2 & 69.1 \\
\hline
\end{tabular}

20 and $150 \mathrm{keV}$. Specialized $\mathrm{x}$-ray tubes used for mammography make use of molybdenum targets and produce lower energy photons between 15 and $30 \mathrm{keV}$. A typical x-ray spectrum from a tungsten target $\mathrm{x}$-ray tube is shown in Figure 2.2. The characteristic peaks of the tungsten target are clearly seen.

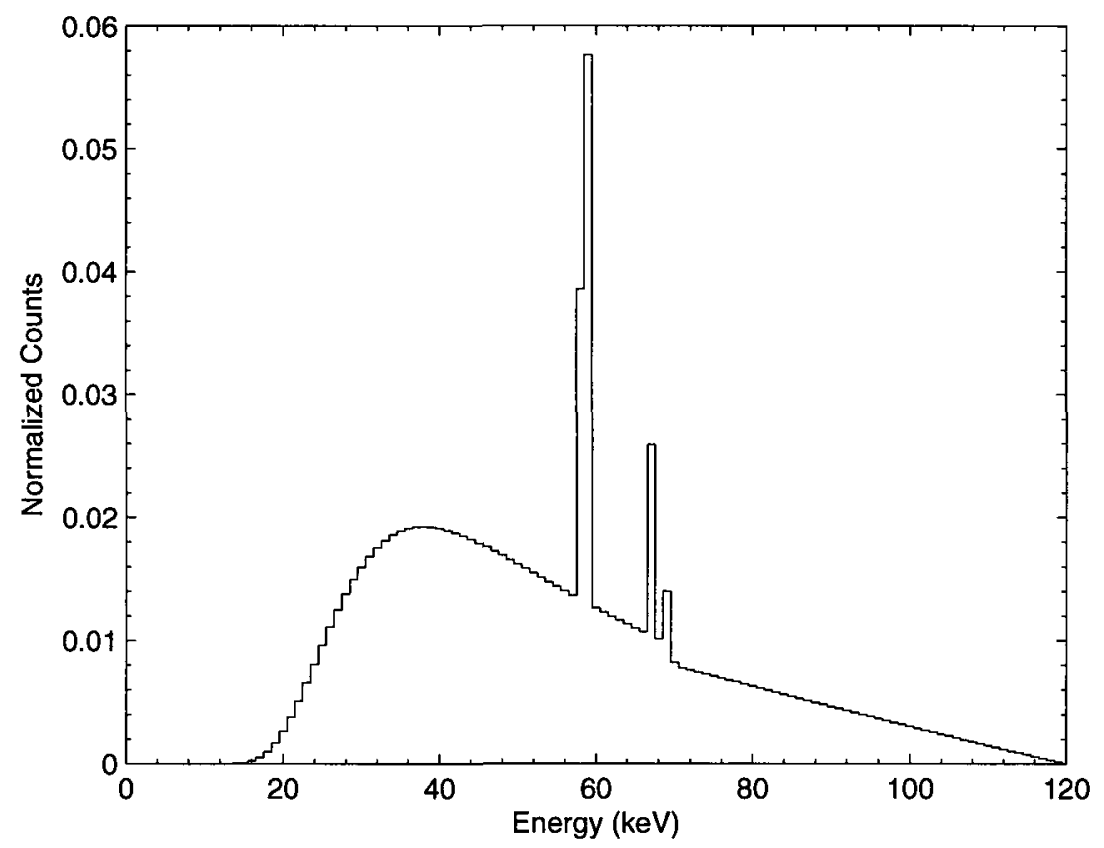

Figure 2.2: Example $x$-ray spectrum from a tungsten target with $120 \mathrm{kV}$ potential and $2.5 \mathrm{~mm}$ of aluminum filtration. Graph generated from data extracted from [34]. 


\subsection{X-Ray Interactions in Matter}

At the energies used in diagnostic radiology, $x$ rays interact with matter in three main ways: the photoelectric effect, coherent scattering and incoherent scattering. At energies above $1.022 \mathrm{MeV}$, pair production is also possible. This is not relevant for the energies of interest in this work and will not be discussed further. The photoelectric effect occurs when a photon transfers all of its energy to an electron, ejecting it from the atom. This was first explained by Albert Einstein, for which he received the Nobel prize in physics in 1921.

In contrast to the photoelectric effect, $\mathrm{x}$-ray photons that are scattered can continue through the target material, carrying additional information. For this reason, the $\mathrm{x}$-ray scattering process will be explored in detail in the following sections.

In scattering experiments, the scattering cross-section $(\sigma)$ is the most important experimentally measurable quantity. The scattering cross-section is defined such that the ratio of the cross-section to the target area is equal to the scattering probability. Measurement of the total cross-section requires a detector completely surrounding the target so what is usually measured is the differential scattering cross-section $(\mathrm{d} \sigma / \mathrm{d} \Omega)$ where $\Omega$ is the solid angle subtended by the detector.

We will treat the scattering of $\mathrm{x}$ rays as two separate processes: coherent scattering, where the $\mathrm{x}$ rays are treated as waves, and incoherent scattering, where the $\mathrm{x}$ rays are treated as relativistic particles.

\subsubsection{Coherent Scattering}

Following the development of Hukins[35], the scattering of $\mathrm{x}$ rays as electromagnetic waves is modelled by considering a monochromatic unpolarized plane wave with wavelength $\lambda$ and amplitude $A_{0}$ incident on a target as shown in Figure 2.3. A detector is placed at an angle $\theta$ a large distance $L$ away from the target. As the electrons in the target are 


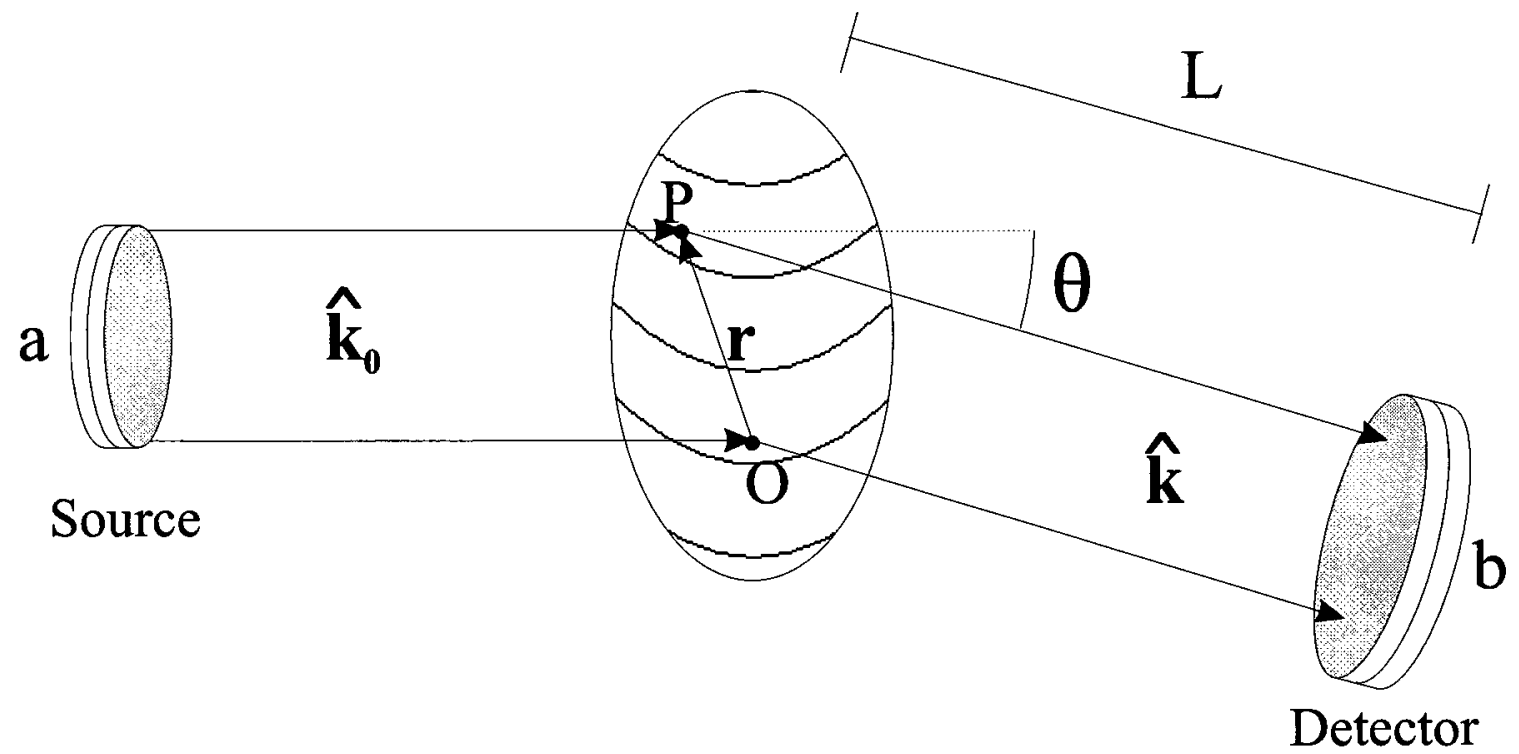

Figure 2.3: Modelling coherent scattering. The variables $a$ and $b$ in the diagram refer to the cross-sectional area of the source and detector respectively.

accelerated by the electromagnetic wave, they create additional wave sources with wavelength $\lambda$ as predicted by classical electrodynamics. For a single electron, the amplitude of the emitted wave at the detector can be shown to be[36]:

$$
A_{s 1}=\frac{A_{0} r_{0}}{L} \sqrt{\frac{1+\cos ^{2} \theta}{2}}
$$

where $r_{0}$ is called the classical electron radius which is defined to be

$$
r_{0}=\frac{1}{4 \pi \varepsilon_{0}} \frac{\mathrm{e}^{2}}{m_{\mathrm{e}} c^{2}}
$$

In this expression, $\mathrm{e}$ is the electron charge, $m_{\mathrm{e}}$ the electron rest mass, $c$ the speed of light, and $\varepsilon_{0}$ the permittivity of free space. The classical electron radius has a numerical value of $2.818 \times 10^{-15} \mathrm{~m}$.

Since the $\mathrm{x}$ rays are being treated as waves, we must take into account interference effects caused by scattering at different locations within the target. Based on Figure 2.3, the difference in path lengths between the source and detector for any two points $\mathrm{O}$ and 
$P$ is given by $\mathbf{r} \cdot \hat{\mathbf{k}}-\mathbf{r} \cdot \hat{\mathbf{k}}_{\mathbf{0}}$ where $\mathbf{r}$ is the position vector joining the points, and $\hat{\mathbf{k}}_{\mathbf{0}}$ and $\hat{\mathbf{k}}$ are unit vectors defining the input and output directions respectively. This gives a phase difference of $\frac{2 \pi}{\lambda}\left(\mathbf{r} \cdot \hat{\mathbf{k}}-\mathbf{r} \cdot \hat{\mathbf{k}}_{\mathbf{0}}\right)$. The amplitude of the emitted wave at the detector can be written as a complex function

$$
\mathrm{d} A_{\mathrm{s}}=\frac{A_{0} \rho_{\mathrm{e}}(\mathbf{r}) r_{0}}{L} \sqrt{\frac{1+\cos ^{2} \theta}{2}} \exp \left[\frac{i 2 \pi}{\lambda}\left(\mathbf{r} \cdot \hat{\mathbf{k}}-\mathbf{r} \cdot \hat{\mathbf{k}}_{\mathbf{0}}\right)\right] \mathrm{d}^{3} \mathbf{r}
$$

where $\rho_{e}(\mathbf{r}) d^{3} \mathbf{r}$ gives the number of electrons at each point with $\rho_{e}(\mathbf{r})$ being the electron density in the target. Integrating this function over the entire target volume gives the total amplitude of the emitted wave at the detector:

$$
A_{\mathrm{s}}=\frac{A_{0} r_{0}}{L} \sqrt{\frac{1+\cos ^{2} \theta}{2}} \int \rho_{\mathrm{e}}(\mathbf{r}) \exp \left\{\frac{i 2 \pi}{\lambda}\left[\mathbf{r} \cdot\left(\hat{\mathbf{k}}-\hat{\mathbf{k}}_{\mathbf{0}}\right)\right]\right\} \mathrm{d}^{3} \mathbf{r}
$$

It can be shown through simple trigonometry that the factor $\frac{2 \pi}{\lambda}\left(\hat{\mathbf{k}}-\hat{\mathbf{k}}_{\mathbf{0}}\right)$ has a magnitude that is dependent on the scattering angle. Specifically:

$$
|\mathbf{s}|=\left|\frac{2 \pi}{\lambda}\left(\hat{\mathbf{k}}-\hat{\mathbf{k}}_{\mathbf{0}}\right)\right|=\frac{4 \pi}{\lambda} \sin \left(\frac{\theta}{2}\right)
$$

Thus, the amplitude can be expressed as a Fourier transform of the target's electron density in the variable s:

$$
A_{\mathrm{s}}=\frac{A_{0} r_{0}}{L} \sqrt{\frac{1+\cos ^{2} \theta}{2}} F_{\mathrm{coh}}(\mathbf{s})
$$

where $F_{\text {coh }}(\mathbf{s})$ is the coherent scattering form factor given by:

$$
F_{\text {coh }}(\mathbf{s})=\int \rho_{\mathrm{e}}(\mathbf{r}) \exp (i \mathbf{r} \cdot \mathbf{s}) \mathrm{d}^{3} \mathbf{r}
$$

Since $F_{\text {coh }}$ is generally a complex function, it is not possible to measure it directly. The best that can be done is to measure the magnitude. Since $x$-ray detectors are sensitive to the power of the emitted wave, which is proportional to the squared amplitude, we calculate 
the power of the incoming and emitted waves:

$$
\begin{gathered}
P_{0}=\frac{c \varepsilon_{0}}{2} A_{0}^{2} a \\
P_{s}=\frac{c \varepsilon_{0}}{2} \frac{A_{0}^{2} r_{0}^{2}}{L^{2}} \frac{\left(1+\cos ^{2} \theta\right)}{2}\left|F_{\mathrm{coh}}(\mathbf{s})\right|^{2} b .
\end{gathered}
$$

From the definition of the input and emitted power, the differential scattering crosssection per solid angle can be expressed as

$$
\frac{\mathrm{d} \sigma_{\mathrm{coh}}}{\mathrm{d} \Omega}=\frac{\mathrm{d}_{\mathrm{e}} \sigma_{0}}{\mathrm{~d} \Omega}\left|F_{\mathrm{coh}}(\mathbf{s})\right|^{2}
$$

where $d_{e} \sigma_{0} / d \Omega$ is the differential scattering cross-section for a single free electron, called the Thomson scattering cross-section:

$$
\frac{\mathrm{d}_{e} \sigma_{0}}{\mathrm{~d} \Omega}=\frac{r_{0}^{2}}{2}\left(1+\cos ^{2} \theta\right)
$$

The form factor and cross-section as developed here are dependent on the size of each particular object through the integration in Equation 2.7. An atomic cross-section and form factor can be calculated by performing the integration over a single atom. The atomic form factor then depends only on the material, not its dimensions. In the case of molecules or mixtures, a molecular form factor could be used instead. Alternatively, a form factor could be calculated per electron. This representation is consistent with any target material. The electronic form factor is related to the atomic form factor by the number of electrons in the atom (atomic number) $Z$ :

$$
\left|F_{\text {coh }}^{\mathrm{a}}\right|^{2}=Z\left|F_{\text {coh }}^{\mathrm{e}}\right|^{2}
$$

The electronic (or atomic) form factor contains the material dependent properties of the scattering process and measuring the form factor is the goal of this thesis. Throughout this thesis, $F_{\text {coh }}$ will refer to electronic form factors only, to avoid confusion. To emphasize 
this, we define the units of $F_{\text {coh }}$ to be (free $\mathrm{e}^{-/}$bound $\left.\mathrm{e}^{-}\right)^{0.5}$, signifying that the form factor converts cross-sections for free electrons into cross-sections for bound electrons. Typically, $F_{\text {coh }}$ is measured in terms of the momentum transfer argument $x$ as defined in Equation 1.1, which is related to $s$ by a factor of $4 \pi$.

\subsubsection{Incoherent Scattering}

The second type of scattering interaction that occurs when $\mathrm{x}$ rays travel through matter is called incoherent scattering. This is based on Compton scattering of the photon. When the photon scatters from a free electron with rest mass $m_{\mathrm{e}}$, the energy and momentum transferred can be calculated directly by conserving the relativistic energy and momentum of both particles. The energy of the scattered photon, $E^{\prime}$ is related to the initial energy $E$ and the scattering angle $\theta$ by the well known expression:

$$
E^{\prime}=\frac{E}{1+\frac{E}{m_{\mathrm{e}} c^{2}}(1-\cos \theta)}
$$

This relationship says nothing, however, about the likelihood of the scattering occurring through any given angle. This likelihood must be calculated through quantum mechanical means. For free electrons, this was done by Klein and Nishina. They showed that the differential cross-section per solid angle $\Omega$ for a photon to scatter from a free electron through angle $\theta$ is given by

$$
\frac{\mathrm{d}_{\mathrm{e}} \sigma_{\mathrm{inc}}}{\mathrm{d} \Omega}=\frac{\mathrm{d}_{\mathrm{e}} \sigma_{0}}{\mathrm{~d} \Omega} \cdot F_{\mathrm{KN}}(E, \theta),
$$

where $F_{\mathrm{KN}}$ is called the Klein-Nishina factor and is given by (see chapter 6.05 of [1])

$$
F_{\mathrm{KN}}(E, \theta)=\left\{\frac{1}{1+\frac{E}{m_{\mathrm{e}} c^{2}}(1-\cos \theta)}\right\}^{2}\left\{1+\frac{\left(\frac{E}{m_{\mathrm{e}} c^{2}}\right)^{2}(1-\cos \theta)^{2}}{\left[1+\frac{E}{m_{\mathrm{e}} c^{2}}(1-\cos \theta)\right]\left(1+\cos ^{2} \theta\right)}\right\}
$$


The Klein-Nishina factor is close to one at diagnostic energies and small scattering angles as shown in Figure 2.4.

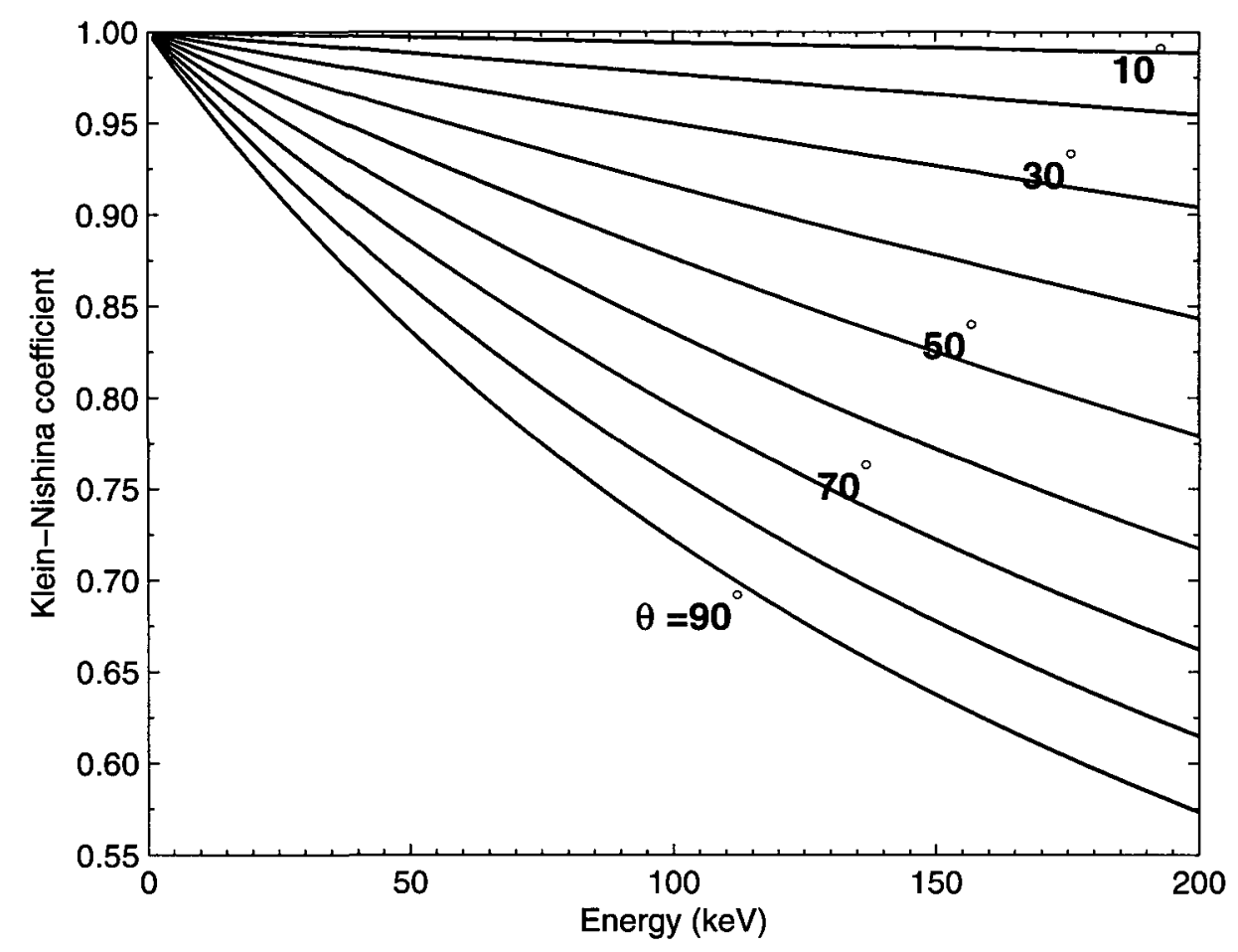

Figure 2.4: Contours of the $F_{\mathrm{KN}}$ function for diagnostic energies.

This description of incoherent scattering is not sufficient for dealing with scattering in real materials as it does not take into consideration the fact that the electrons are bound to atoms. The effect of the atomic binding is generally dealt with by computing the probability that the electron, having been scattered incoherently according to Equation 2.14 with energy $E-E^{\prime}$, would escape from the atom. In the low energy region, this probability is a function of the momentum transfer argument, $x$ and the probability of the electron escaping from the atom is called the incoherent scattering form factor, $F_{\text {inc }}$. The incoherent scattering form factor for individual atoms can be calculated quantum mechanically[17] and combined to determine $F_{\text {inc }}$ for compounds and mixtures. The differential incoherent scattering cross-section per atom per steradian for any material is then given by

$$
\frac{\mathrm{d}_{\mathrm{a}} \sigma_{\mathrm{inc}}}{\mathrm{d} \Omega}=\frac{\mathrm{d}_{\mathrm{e}} \sigma_{0}}{\mathrm{~d} \Omega} \cdot F_{\mathrm{KN}}(E, \theta) F_{\mathrm{inc}}^{\mathrm{a}}(x)
$$


where $F_{\text {inc }}^{\mathrm{a}}$ is the atomic incoherent scattering form factor. Equivalently, the scattering cross-section per electron per steradian can be written in terms of the electronic incoherent scattering form factor $F_{\text {inc }}^{\mathrm{e}}$ :

$$
\frac{\mathrm{d}_{\mathrm{e}} \sigma_{\mathrm{inc}}}{\mathrm{d} \Omega}=\frac{\mathrm{d}_{\mathrm{e}} \sigma_{0}}{\mathrm{~d} \Omega} \cdot F_{\mathrm{KN}}(E, \theta) F_{\mathrm{inc}}^{\mathrm{e}}(x)
$$

where, analogous to the coherent scattering form factor, the atomic and electronic form factors are related by:

$$
F_{\text {inc }}^{\mathrm{a}}=Z F_{\text {inc }}^{\mathrm{e}} .
$$

Electronic incoherent scattering form factors are referred to exclusively in this thesis as $F_{\text {inc }}$ to avoid confusion. The incoherent scattering form factors for some common materials are shown in Figure 2.5. Different materials have very similar values of $F_{\text {inc }}$, suggesting that the image quality improvement from incoherent scattering may be limited.

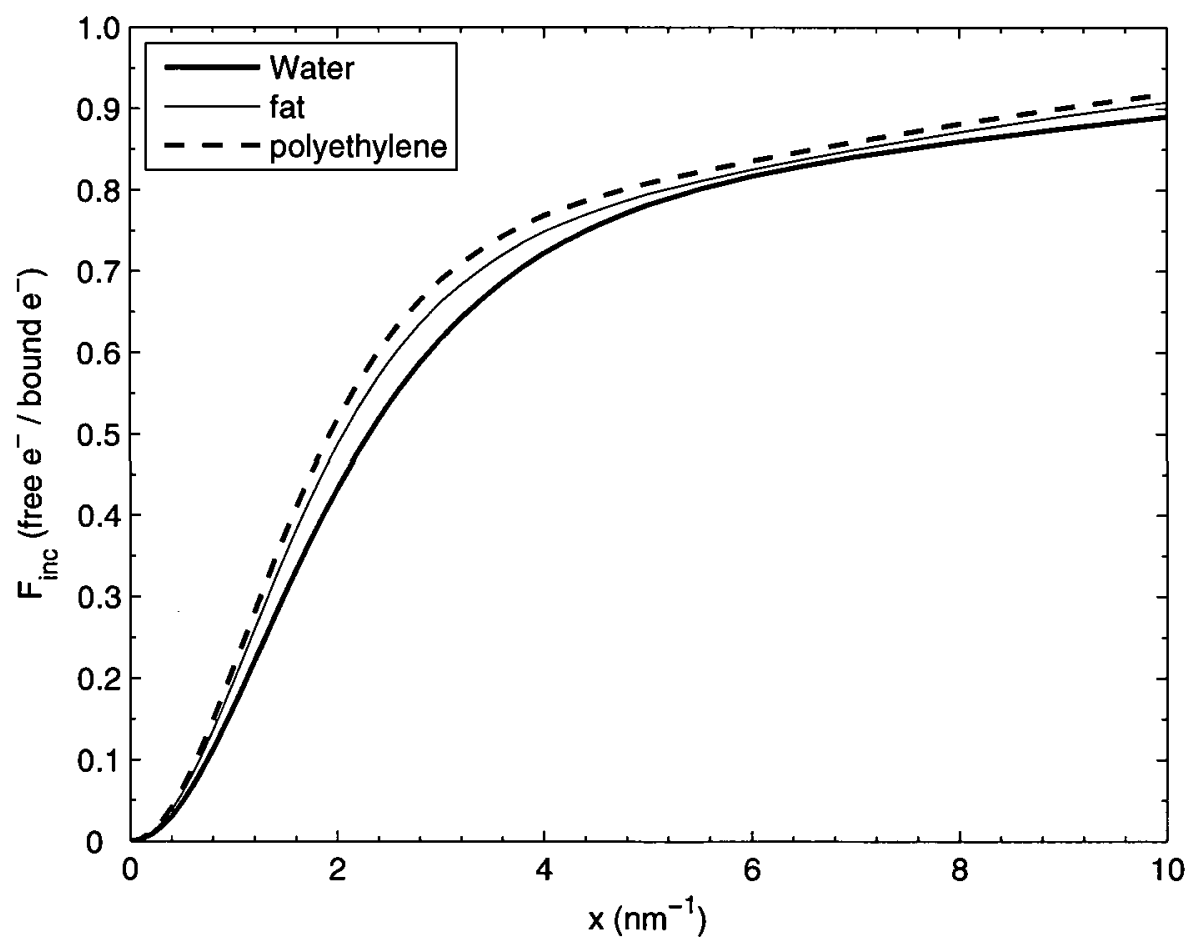

Figure 2.5: Calculated values of $F_{\text {inc }}$ based on atomic form factors calculated from Reference [17]. 


\subsubsection{Total Scattering}

Based on Equations 2.10 and 2.17 the total differential scattering cross-section per electron per steradian can be seen to be the sum of the coherent and incoherent scattering crosssections, as follows:

$$
\frac{\mathrm{d}_{\mathrm{e}} \sigma}{\mathrm{d} \Omega}=\frac{\mathrm{d}_{\mathrm{e}} \sigma_{0}}{\mathrm{~d} \Omega}\left[F_{\mathrm{coh}}^{2}(x)+F_{\mathrm{KN}}(E, \theta) F_{\mathrm{inc}}(x)\right] .
$$

The coherent and incoherent form factors for individual atoms have been calculated based on quantum mechanical modelling[17]. These calculations show that coherent scattering is strongly forward directed while incoherent scattering is suppressed in the forward direction. The fraction of photons scattered at angle $\theta$ that underwent coherent scattering as calculated for independent water molecules is shown in Figure 2.6. This suggests that for diagnostic energies, coherent scattering can most easily be observed in water and tissues for scattering angles smaller than $\sim 15^{\circ}$.

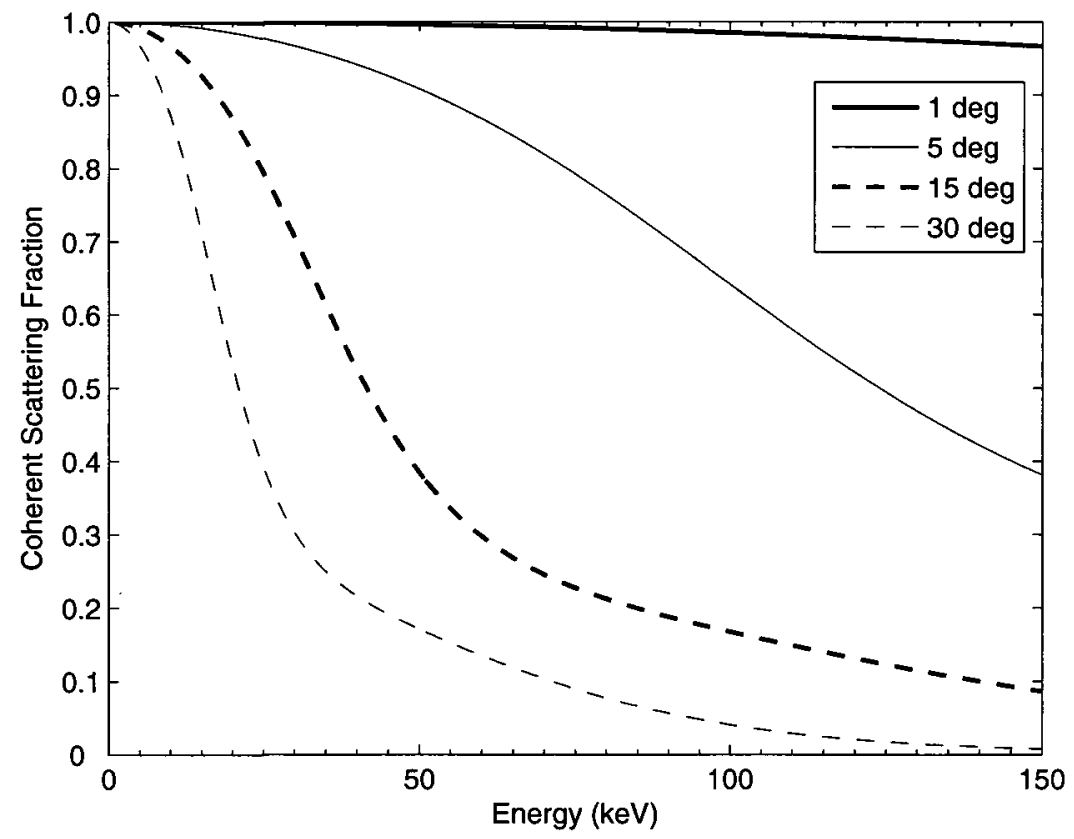

Figure 2.6: Coherent scatter fraction for water at different scattering angles calculated using the independent atom model. (Based on data from Reference [17]) 


\subsection{Detector Technology}

Here I discuss the theory behind several common methods of detecting $\mathrm{x}$ rays. The most basic method of detecting $\mathrm{x}$ rays is through the use of a photographic emulsion. This was the method used by Roentgen in his initial discovery. X rays, like other types of electromagnetic radiation, cause a chemical reaction to occur in a photographic emulsion. They do this by aiding in the reduction of silver ions to metallic silver in the emulsion. Because the size of this effect is dependent on the total energy deposited by the $x$ rays, film is considered an energy integrating $\mathrm{x}$-ray detector. This is very different from other types of x-ray detectors such as Geiger counters which count individual photons. As a practical limitation, this means that $\mathrm{x}$-ray film and other energy integrating detectors do not contain any information regarding the energy of the individual photons.

Most x-ray film systems use a combination of a fluorescence screen and film together in a cassette. The $\mathrm{x}$ rays excite visible light fluorescence photons in the screen which are then absorbed by the film emulsion to create the image. Direct stimulation of the $\mathrm{x}$-ray film is not very efficient because the very thin film does not absorb a significant number of high energy $x$ rays.

\subsubsection{Storage Plates}

A newer development in the area of $\mathrm{x}$-ray imaging is the digital acquisition of images using storage plates made of photo-stimulable phosphors. These storage plates are packaged in a cassette, similar to a screen/film combination, but the images can be read out digitally and the plate re-used multiple times. Storage plates do not need a fluorescence screen to aid image formation. Instead of causing a chemical reaction, $x$ rays cause electrons in the plate to jump to a higher energy level. This higher level is a trapped state, meaning that the electron can remain in this excited state for long periods of time. The number of electrons in the excited state is proportional to the energy absorbed by the storage plate. The image 
is read by exposing the screen to a laser source that has enough energy to excite electrons from the trapped state to the conduction band without causing additional valence band excitations. When these conduction electrons decay to the valence band, visible light of a particular wavelength is emitted. The amount of this light generated represents the signal. This process is displayed in Figure 2.7.

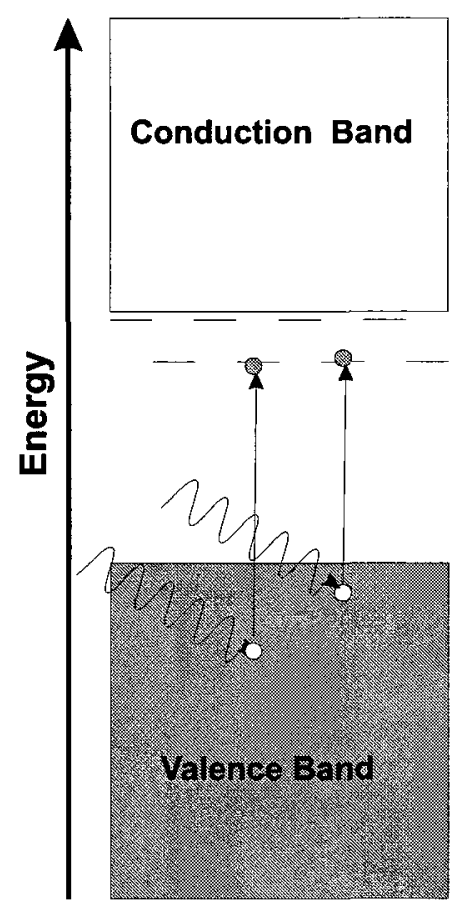

X-ray Exposure

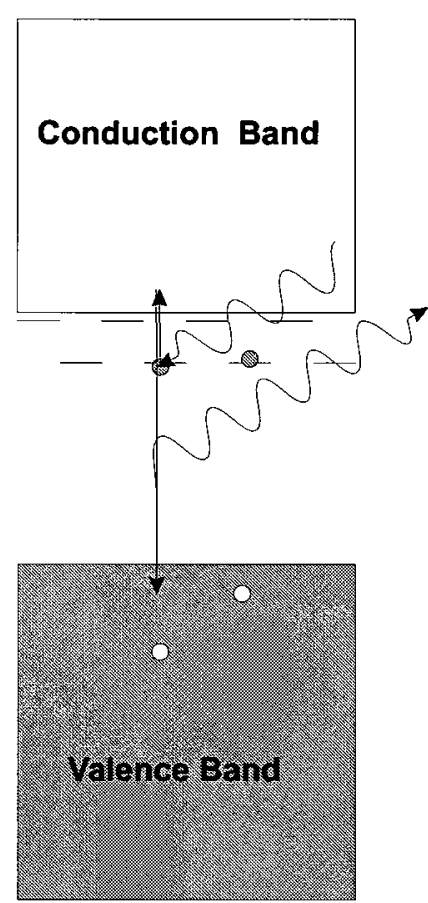

Readout

Figure 2.7: Schematic representation of the image acquisition process in photo-stimulable phosphor plates.

Since $\mathrm{x}$ rays can penetrate some distance through a substance without interacting and storage plates are quite thin, only a portion of the energy of the x-ray beam is absorbed by the detector. There are two factors that determine the strength of the signal for a given photon of energy $E$. The first is the probability that the photon will interact with the plate as it passes through. This is the quantum efficiency of the plate which can be calculated if the composition is known. If we refer to the interaction probability as $p_{\mathrm{d}}(E)$, then this is simply:

$$
p_{\mathrm{d}}(E)=1-e^{-\mu(E) t}
$$


where $\mu$ is the attenuation coefficient of the screen and $t$ is the thickness. This can be measured directly using an energy sensitive detector and a source of $\mathrm{x}$ rays simply by measuring the number of photons of a given energy that are observed with and without the screen present.

The second factor that determines the strength of the signal is the signal conversion factor, $s_{\mathrm{d}}(E)$. This is the signal generated by a single photon interacting with the detector. For an ideal energy integrating detector, this should be proportional to the energy of the photon. In an actual detector, averaged over many interactions, some of the energy would escape from the detector through fluorescence or Compton scattering. This can be quantified in terms of the Swank factor[37]. We will treat the phosphor screen as an ideal energy integrator for simplicity.

\subsubsection{Ionization Chambers}

An ionization chamber measures the amount of ionization caused by $\mathrm{x}$ rays passing through a volume of air. This is proportional to the energy deposited by the beam. Ionization chambers are typically used to measure absorbed dose (energy deposited per unit mass of material) in clinical applications. Absorbed dose depends in a complicated manner on a number of factors including the exposure parameters, type of material and field size. Ionization chambers interact only weakly with the x-ray beam because they contain mostly air, meaning that they can be used directly in an x-ray beam as monitor chambers, measuring the relative strength of an $\mathrm{x}$-ray beam without significantly affecting it.

\subsubsection{Germanium Spectrometers}

In some cases, it is important to measure the energies of the photons, not their spatial location. An x-ray spectrometer is useful in these situations. A High Purity Germanium (HPGe) spectrometer consists of a very pure crystal of germanium with electrical contacts on either end. The crystal is chilled using either liquid nitrogen or a mechanical cooler[32] 
to reduce the effect of thermal fluctuations. Since germanium is a semiconductor, at low temperatures essentially all of the electrons in the crystal will be in the semiconductor valence band with a band gap of a few electron-Volts between the valence band and conduction band. When an x-ray photon passes through the crystal, it excites some of the electrons across the band gap into the conduction band. The number of electrons excited will be proportional to the energy absorbed in the crystal. These conduction electrons are swept out of the crystal by a large bias voltage giving a charge pulse proportional to the photon energy. This process is shown schematically in Figure 2.8 .

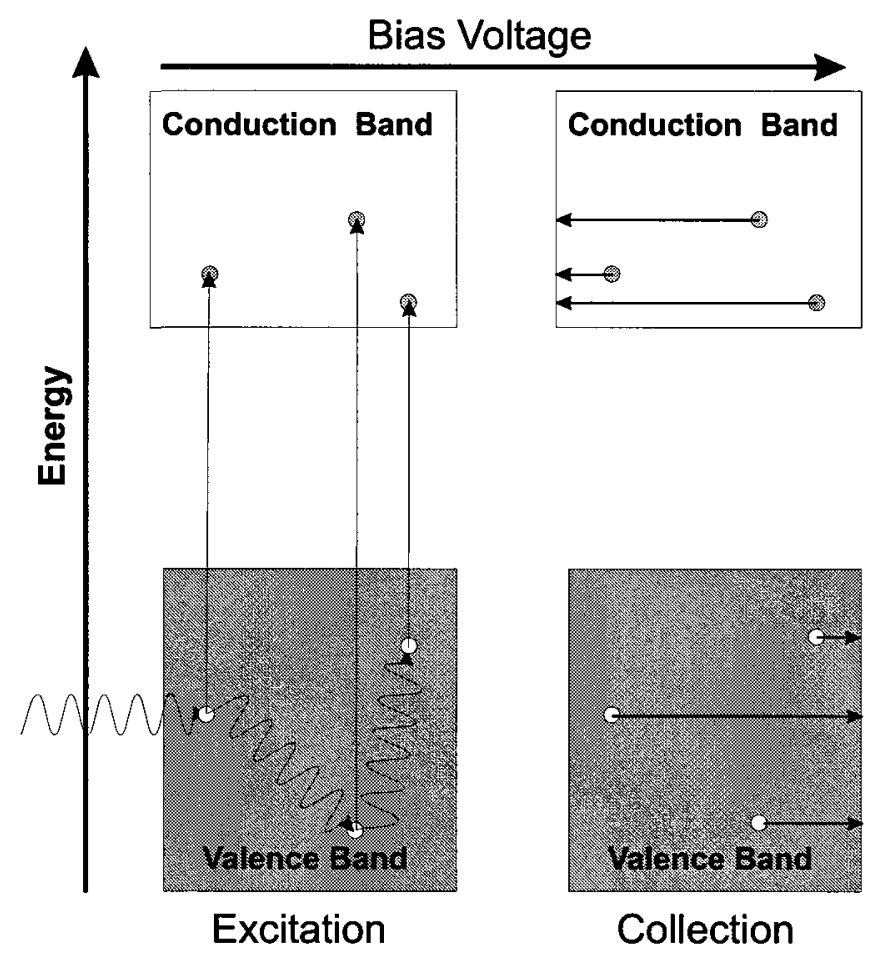

Figure 2.8: Schematic representation of HPGe spectrometer operation.

In an ideal spectrometer, all of the photon's energy is absorbed within the detector crystal. Measuring the energy spectrum of a monochromatic source of $x$ rays with such an ideal detector would show a sharp delta function representing the photon's energy as illustrated in Figure 2.9. In practice, however, this is not observed because in some cases, the photon energy is only partially absorbed in the detector. This causes a range of energies lower than the actual energy to be observed in the measured spectrum. The observed 


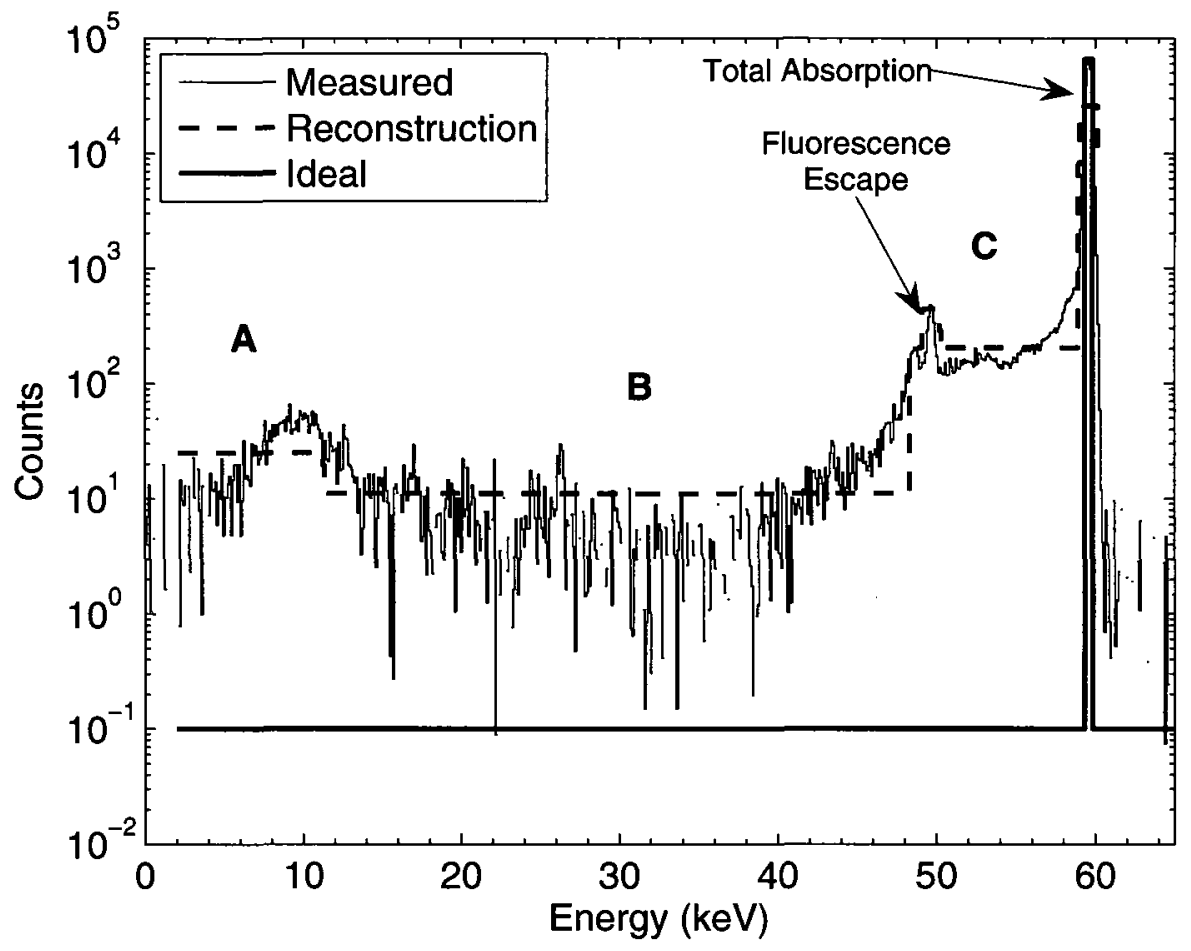

Figure 2.9: $X$-ray spectrum of monochromatic source (Am-241) as would be measured with an ideal spectrometer compared to that measured with our HPGe spectrometer and the reconstructed spectrum as used in measuring the detector response function (Spectrum measured by Charles Steiner). A, B and $\mathrm{C}$ represent the Compton, multiple and backreflection energy regions respectively.

spectrum can be used to characterize the detector response function of the detector which is necessary in order to correct for this effect. We have developed a method similar to that in Reference [38] that is based on analyzing the different types of interactions that can occur when an $\mathrm{x}$-ray photon interacts with the detector crystal. These interactions are illustrated in Figure 2.10 and their energy ranges can be defined as follows:

1. Total Absorption: The entire energy of the photon is absorbed in the detector. This may occur in several interactions but none of the energy of the original photon escapes from the crystal. This is responsible for the full energy peak in the spectrum.

2. Compton Scattering: If the photon scatters inelastically in the crystal through the Compton effect, the scattered photon can escape from the crystal. Since the energy 


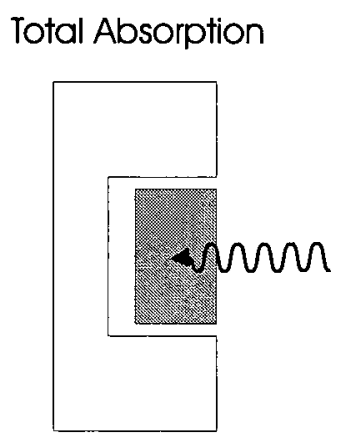

Compton Scatter

Fluorescent Escape

Backreflection

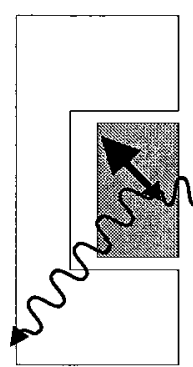

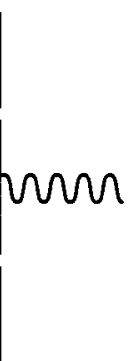

Multiple Scatter
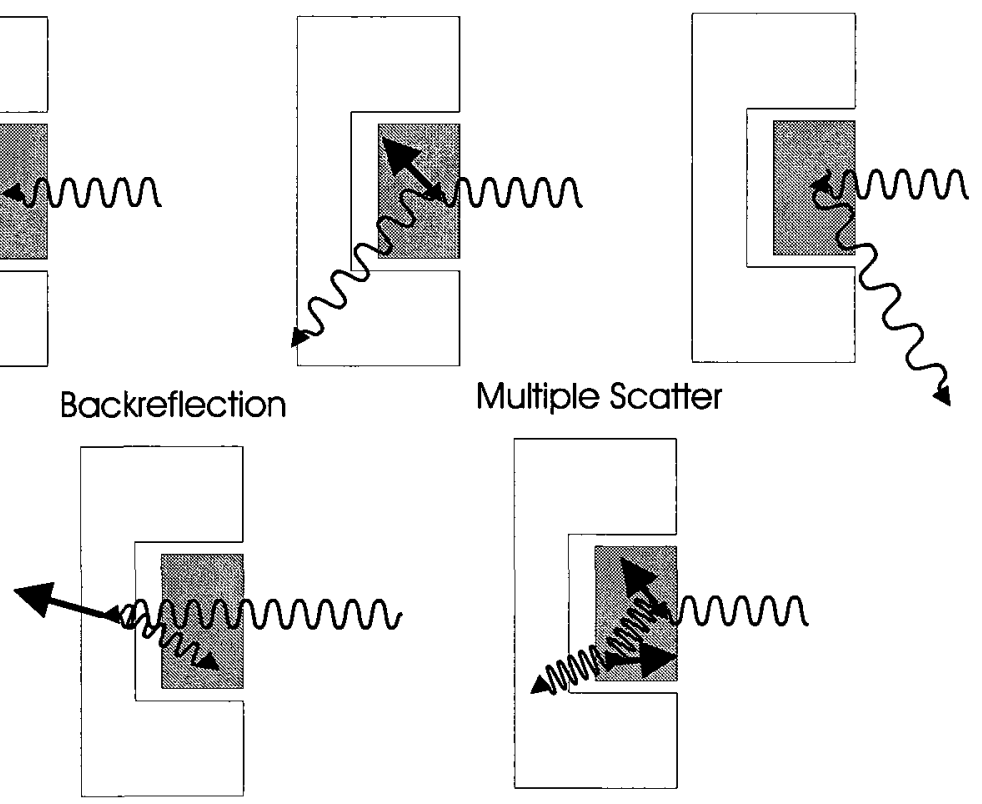

Figure 2.10: $\mathrm{X}$-ray interaction processes occurring within a HPGe detector.

of the scattered photon depends on the scattering angle, a range of energies can be lost. This gives rise to a low energy plateau in the measured spectrum. The upper limit of this plateau can be determined by calculating the energy lost when the photon scatters through the maximal angle, 180 degrees. Using the Compton scattering formula from Equation 2.13 gives the energy of the Compton Edge $E_{\mathrm{CE}}$ for a photon of energy $E$ :

$$
E_{\mathrm{CE}}=E-\frac{E}{1+\frac{2 E}{m_{\mathrm{e}} c^{2}}},
$$

which is $11.25 \mathrm{keV}$ for the $59.537 \mathrm{keV}$ photon of Am-241.

3. Fluorescence Escape: The $x$-ray photon excites a fluorescence photon in the germanium by ejecting an inner shell electron. If this fluorescence photon escapes from the crystal, its energy is not recorded with the pulse. Germanium gives fluorescence lines at approximately $9.9 \mathrm{keV}$ so the fluorescence escape interaction causes an energy $9.9 \mathrm{keV}$ lower than the total energy.

4. Backreflection Scattering: If the photon scatters inelastically outside of the crystal 
before being absorbed by the detector, only the energy of the scattered photon is measured. This gives rise to a plateau similar to that of Compton Scattering but in the range from the backreflection edge $E_{\mathrm{BE}}$ up to the full energy peak:

$$
E_{\mathrm{BE}}=\frac{E}{1+\frac{2 E}{m_{\mathrm{e}} c^{2}}},
$$

which is $48.29 \mathrm{keV}$ for the $59.537 \mathrm{keV}$ photon of Am-241.

5. Multiple Scattering: If the photon undergoes more than one interaction that allows energy to escape from the crystal, the measured energy could be recorded anywhere in the spectrum. We define the energy range for this interaction to be anywhere between the Compton and backreflection plateaus.

The detector response function can then be written with contributions from each type of interaction as illustrated in Figure 2.9 and these values can be interpolated to give the detector response function at any energy. The contribution from each process is shown in Figure 2.11. This is a fairly crude reconstruction that does not capture the fine detail present in the measured spectrum. Nevertheless, it provides a reasonably accurate detector correction in most cases.

We have measured detector response functions for our HPGe spectrometer using several monochromatic sources as listed in Table 2.2. The filters were used to isolate particular energies when more than one were present. Detector correction is performed in the MCASpectrum class (see Appendix C.3) by an iterative function called DetectorCorrection. The detector correction is a small effect for $\mathrm{x}$-ray tube spectra except around the low energy cut-off where an extra plateau is observed as shown in Figure 2.12. This method is used in the angle-dispersive measurements of Chapters 3 and 4 but was found to be unnecessary for the subsequent energy-dispersive work in Chapters 5 and 6. 


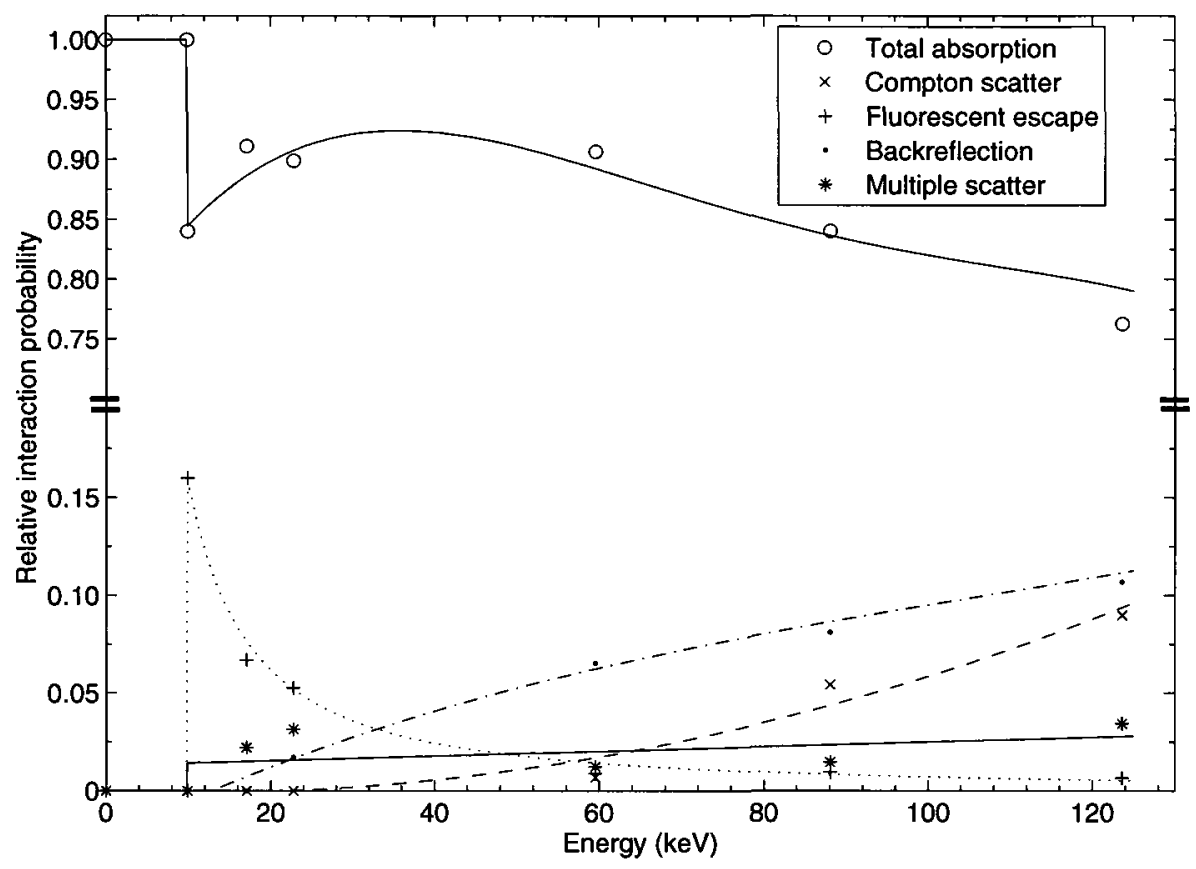

Figure 2.11: Contribution of different processes to detector response function. The lines show the interpolating functions used in each case. Note the change in scale on the y-axis. Measurements were made by Charles Steiner.

Table 2.2: Monochromatic sources used to measure detector response function of the HPGe detector. The energies were taken from Appendix 2 of Reference [33]. In some cases, a weighted average of two closely spaced energies were used. To isolate the appropriate monochromatic energy, a reference spectrum (with appropriate filtration) was subtracted from the source spectrum (with appropriate filtration). Measurements were made by Charles Steiner.

\begin{tabular}{lllll}
\hline Source & Filter & Reference & Filter & Energy $(\mathrm{keV})$ \\
\hline \hline $\mathrm{Co}-57$ & $\mathrm{Cu}, \mathrm{Al}$ & background & $\mathrm{Cu}, \mathrm{Al}$ & 123.6 \\
$\mathrm{Cd}-109$ & $\mathrm{Cu}, \mathrm{Al}$ & background & $\mathrm{Cu}, \mathrm{Al}$ & 88.0 \\
$\mathrm{Am}-241$ & $\mathrm{Cu}, \mathrm{Al}$ & background & $\mathrm{Cu}, \mathrm{Al}$ & 59.5 \\
$\mathrm{Am}-241$ & $\mathrm{Zr}$ & $\mathrm{Am}-241$ & $\mathrm{Cu}, \mathrm{Al}$ & 17.8 \\
$\mathrm{Cd}-109$ & $\mathrm{Ag}$ & $\mathrm{Cd}-109$ & $\mathrm{Cu}, \mathrm{Al}$ & 22.7 \\
\hline
\end{tabular}




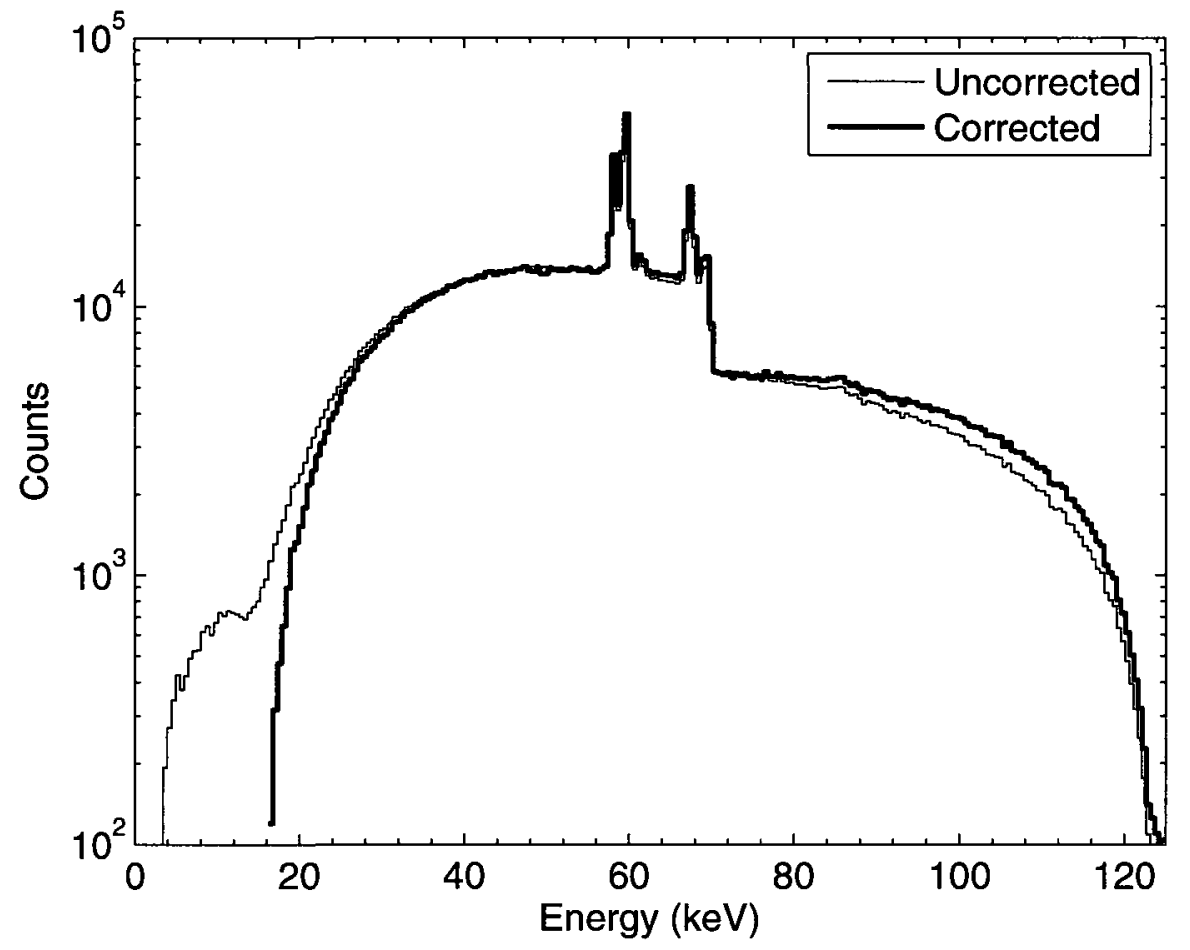

Figure 2.12: Effect of detector correction on measured tungsten anode $\mathrm{x}$-ray spectrum $(121 \mathrm{kVp}, 2 \mathrm{~mA})$. Measurements were made by Charles Steiner. 


\section{CHAPTER 3}

\section{ANGLE-DISPERSIVE FORM FACTOR}

\section{MEASUREMENTS USING IMAGING}

\section{DETECTORS - DEVELOPMENT}

Measurement of the coherent scattering form factor can be accomplished in several ways. In this chapter, I develop an angle-dispersive technique, measuring at a series of scattering angles. Traditional angle-dispersive techniques use a monochromator to pick out a particular energy of $\mathrm{x}$-ray photons. I show here that it is possible to measure the form factor without a monochromator in some cases if the spectral characteristics of the source are known. This makes more efficient use of the polychromatic nature of a typical $\mathrm{x}$-ray tube.

\subsection{Model Development}

The experimental setup that will be modelled is shown in Figure 3.1. The detector is an energy insensitive, large area detector as described in Section 2.3.1. This could be a screen-film system, image intensifier, storage phosphor plate or other type of imaging detector. The system is cylindrically symmetric about the $\mathrm{x}$-ray pencil beam such that the scattering can be described simply in terms of the angle $\theta$. For this system, assuming that $L_{\mathrm{t}} \ll d_{\mathrm{t}}$ such that the variation in scatter angle throughout the target is small and ignoring 


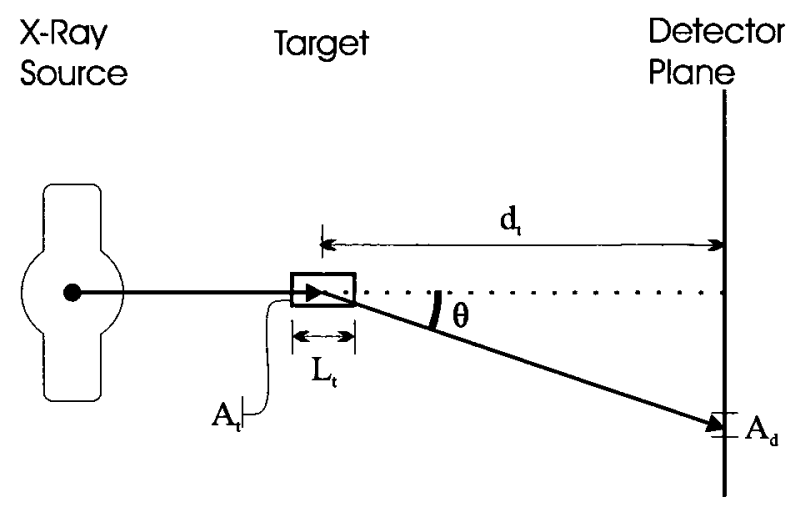

Figure 3.1: The ideal experimental layout that has been modelled for an angle dispersive form factor measurement using imaging detectors. The $\mathrm{x}$ ray source is collimated down to a pencil beam. The cross-sectional areas of the target and the region in the detector plane are given by $A_{\mathrm{t}}$ and $A_{\mathrm{d}}$, respectively.

the effect of multiple scatter, the scatter signal measured by the detector from photons with energy $E$ scattering through angle $\theta$ at depth $l$ in the target is simply the product of the number of x-ray photons incident on the target, the fraction that scatter through a given angle and reach the detector, and the signal generated by the detector by a given photon:

$$
\mathrm{d} S(E, \theta, l)=\left[\frac{\mathrm{d} \Phi}{\mathrm{d} E} A_{\mathrm{t}} \mathrm{d} E\right]\left[e^{-\mu_{\mathrm{t}}(E) l}\right]\left[\frac{\mathrm{d}_{e} \sigma}{\mathrm{d} \Omega} \rho_{\mathrm{e}} \mathrm{d} l\right]\left[e^{-\mu_{\mathrm{t}}(E)\left(L_{\mathrm{t}}-l\right)}\right]\left[\frac{A_{\mathrm{d}} \cos ^{3} \theta}{d_{\mathrm{t}}^{2}} p_{\mathrm{d}}(E) s_{\mathrm{d}}(E)\right] .
$$

In this equation, $\mathrm{d} \Phi / \mathrm{d} E$ is the differential photon fluence from the x-ray source, $\mu_{\mathrm{t}}$ is the target's attenuation coefficient and $\rho_{\mathrm{e}}$ is the electron density of the target. The functions $p_{\mathrm{d}}(E)$ and $s_{\mathrm{d}}(E)$ were defined in Section 2.3.1. For small scattering angles such that the distance travelled through the target is $\sim L_{\mathrm{t}}$, integrating this equation over all depths in the target and all energies in the spectrum gives

$$
S(\theta)=\frac{\rho_{\mathrm{e}} A_{\mathrm{t}} A_{\mathrm{d}} L_{\mathrm{t}} \cos ^{3} \theta}{d_{\mathrm{t}}^{2}} \int_{E_{\min }}^{E_{\max }} e^{-\mu_{\mathrm{t}}(E) L_{\mathrm{t}}} \frac{\mathrm{d} \Phi}{\mathrm{d} E} \frac{\mathrm{d}_{\mathrm{e}} \sigma}{\mathrm{d} \Omega} \mathrm{p}_{\mathrm{d}}(E) s_{\mathrm{d}}(E) \mathrm{d} E .
$$

Inserting the definition for the scattering cross section from Equation 2.19 leads to an 
integral equation involving the coherent scattering form factor:

$$
S(\theta)=\chi(\theta) \int_{E_{\min }}^{E_{\max }} \frac{\mathrm{d} P}{\mathrm{~d} E}\left[F_{\mathrm{coh}}^{2}(x)+F_{\mathrm{KN}}(E, \theta) F_{\mathrm{inc}}(x)\right] \mathrm{d} E,
$$

where

$$
\chi(\theta)=\left[\frac{r_{0}^{2}\left(1+\cos ^{2} \theta\right)}{2}\right]\left[\frac{\rho_{\mathrm{e}} A_{\mathrm{t}} A_{\mathrm{d}} L_{\mathrm{t}} \cos ^{3} \theta}{d_{\mathrm{t}}^{2}}\right]
$$

and $\mathrm{d} P / \mathrm{d} E$ is the detector signal as a function of energy for the primary beam transmitted through the sample:

$$
\frac{\mathrm{d} P}{\mathrm{~d} E}=e^{-\mu_{\mathrm{t}}(E) L_{\mathrm{t}}} \frac{\mathrm{d} \Phi}{\mathrm{d} E} p_{\mathrm{d}}(E) s_{\mathrm{d}}(E) .
$$

All of the quantities in Equation 3.3 are known (to a scaling constant) with the exception of the coherent scattering form factor. Because $F_{\mathrm{inc}}(x)$ is known from the data of Hubbell and Øverbø [17], the second term in the integral can be calculated and removed. This leaves an equation of the form:

$$
S^{\prime}(\theta)=\chi(\theta) \int_{E_{\min }}^{E_{\max }} \frac{\mathrm{d} P}{\mathrm{~d} E} F_{\text {coh }}^{2}(x) \mathrm{d} E
$$

where $S^{\prime}$ contains the known incoherent contribution to the integral. Explicitly:

$$
S^{\prime}(\theta)=S(\theta)-\chi(\theta) \int_{E_{\min }}^{E_{\max }} \frac{\mathrm{d} P}{\mathrm{~d} E} F_{\mathrm{KN}}(E, \theta) F_{\mathrm{inc}}(x) \mathrm{d} E
$$

For small scattering angles, the incoherent contribution to $S^{\prime}$ is negligible. However, Figure 2.6 shows that, for water with $40 \mathrm{keV}$ photons and 15 degree scattering angle, the contributions are roughly equal. For larger energies or angles, the incoherent contribution dominates.

By converting the integration over energy in Equation 3.6 into an integration over $x$ via 
Equation 1.1, a simpler relationship is obtained,

$$
S^{\prime}(\theta)=\chi(\theta) \int_{x_{\min }}^{x_{\max }} \frac{\mathrm{d} P}{\mathrm{~d} x} F_{\text {coh }}^{2}(x) \mathrm{d} x
$$

The $\mathrm{d} P / \mathrm{d} x$ function scales the energy dependent $\mathrm{d} P / \mathrm{d} E$ into $x$-space based on the known value of $\theta$. If a series of measurements are made at $m$ different scattering angles and $F_{\text {coh }}$ is to be measured at $n$ different points, a series of integral equations is produced. Writing these in discrete form gives a matrix equation:

$$
\left[\begin{array}{c}
S_{1}^{\prime} \\
S_{2}^{\prime} \\
\vdots \\
S_{m}^{\prime}
\end{array}\right]=\left[\begin{array}{cccc}
\chi\left(\theta_{1}\right) P_{\mathrm{x}}\left(\theta_{1}, x_{1}\right) & \chi\left(\theta_{1}\right) P_{\mathrm{x}}\left(\theta_{1}, x_{2}\right) & \cdots & \chi\left(\theta_{1}\right) P_{\mathrm{x}}\left(\theta_{1}, x_{n}\right) \\
\chi\left(\theta_{2}\right) P_{\mathrm{x}}\left(\theta_{2}, x_{1}\right) & \chi\left(\theta_{2}\right) P_{\mathrm{x}}\left(\theta_{2}, x_{2}\right) & \cdots & \chi\left(\theta_{2}\right) P_{\mathrm{x}}\left(\theta_{2}, x_{n}\right) \\
\vdots & \vdots & \ddots & \vdots \\
\chi\left(\theta_{m}\right) P_{\mathrm{x}}\left(\theta_{m}, x_{1}\right) & \chi\left(\theta_{m}\right) P_{\mathrm{x}}\left(\theta_{m}, x_{2}\right) & \cdots & \chi\left(\theta_{m}\right) P_{\mathrm{x}}\left(\theta_{m}, x_{n}\right)
\end{array}\right]\left[\begin{array}{c}
F_{\mathrm{coh}}^{2}\left(x_{1}\right) \\
F_{\mathrm{coh}}^{2}\left(x_{2}\right) \\
\vdots \\
F_{\mathrm{coh}}^{2}\left(x_{n}\right)
\end{array}\right],
$$

which can be written more concisely as

$$
\mathbf{S}^{\prime}=\underline{\chi} \underline{\mathbf{P}_{\mathbf{x}}} \mathbf{F}_{\text {coh }}^{2}
$$

In this equation, $\underline{\chi}$ is an $m \times m$ diagonal matrix containing $\chi(\theta)$ along the diagonal and $\underline{\mathbf{P}}_{\mathbf{x}}$ is an $m \times n$ matrix containing the $\mathrm{d} P / \mathrm{d} x$ function. In principle, equation 3.10 can be solved simply by inverting the $\underline{\mathbf{P}_{\mathbf{x}}}$ matrix. In practice, however, this matrix is ill-conditioned for typical $x$-ray spectra. The conditioning of a matrix system is determined by its condition number (see chapter 3 of Reference [39]). Condition numbers close to one represent a well-conditioned system. Condition numbers of the $\underline{\mathbf{P}_{\mathbf{x}}}$ matrix are on the order of $10^{10}$. Alternative methods need to be used to extract $F_{\text {coh }}$ from the equation. Two methods to do this will be developed in the next section. 


\subsection{Mathematical Methods}

\subsubsection{Effective Energy Approximation}

For reference, we first note how $F_{\text {coh }}$ can be calculated assuming that all scattering occurred at the same effective energy. By assuming a delta function for $\mathrm{d} \Phi / \mathrm{d} E$, the integration over energy in Equation 3.3 is removed. There is then a one to one correspondence between the measured scattering and the coherent scattering form factor at a given $x$ value. In terms of the matrix representation, this assumption reduces $\underline{\mathbf{P}_{\mathbf{x}}}$ to a diagonal form, which is trivially invertible and well conditioned by definition. Standard angle-dispersive diffractometers use a crystal monochromator to isolate a characteristic x-ray peak from the spectrum.

Using an effective energy approximation in our experiment, however, is much less precise due to the wider range of energies in each $\mathrm{x}$-ray beam. This approximation results in a form factor that is smeared in $x$-space. The methods developed in the following section make use of the spectral information to generate a better estimate of the coherent scattering form factor. In both methods, detailed knowledge of the $\mathrm{x}$-ray spectrum is required to determine $\mathrm{d} P / \mathrm{d} E$ from Equation 3.5.

\subsubsection{Sub-matrix Calculation}

In an effort to improve the conditioning of the matrix equation, we note that there are no conditions on the particular $x$ values used in $\mathbf{F}_{\text {coh }}$ in Equation 3.10. We are free to choose any values for $x$ as long as they cover the required range between $x_{\min }$ and $x_{\max }$. We are also free to use only a subset of the $\mathbf{S}^{\prime}$. By making use of this freedom, we can construct a series of smaller sub-matrices that are each diagonally dominant. This means that the element on the diagonal in each row is larger than the sum of all other elements in the row. The advantage of diagonally dominant matrices is that they are generally well- 
conditioned[40]. An algorithm to construct one of these sub-matrices is given below. The sub-matrix elements will be at angles $\Theta_{k}$ and momentum transfer values $X_{j}$. Each $X_{j}$ is at the midpoint between $X_{j}^{\min }$ and $X_{j}^{\max }$.

1. From the known $\mathrm{d} P / \mathrm{d} E$ function, find the minimum, median and maximum energies, $E_{\min }, E_{\mathrm{med}}, E_{\mathrm{max}}$.

2. For the smallest angle $\theta_{i}$ not used in any other sub-matrices, convert $E_{\min }, E_{\mathrm{med}}$, $E_{\max }$ to $x_{\min }, x_{\operatorname{med}}, x_{\max }$ via Equation 1.1.

3. Because the sub-matrix must be a lower triangular matrix, for the first row $(k=1)$ of the sub-matrix, the entire $\mathrm{d} P / \mathrm{d} x$ function must be located in the first column $(j=1)$. This is arranged by setting $X_{1}^{\min }=x_{\min }, X_{1}^{\max }=x_{\max }, \Theta_{1}=\theta_{i}$ and increment $j$ and $k$.

4. For the next smallest angle $\theta_{i}$ not used in any other sub-matrices, convert $E_{\min }, E_{\mathrm{med}}$, $E_{\max }$ to $x_{\min }, x_{\operatorname{med}}, x_{\max }$ via Equation 1.1.

5. Compare $x_{\text {med }}$ to $X_{j-1}^{\max }$. If $x_{\text {med }}>X_{j-1}^{\max }$, the diagonal element will be the dominant member of the row and this angle can be used in constructing the diagonally dominant sub-matrix. Set $X_{j}^{\min }=X_{j-1}^{\max }, X_{j}^{\max }=x_{\max }, \Theta_{k}=\theta_{i}$ and increment $j$ and $k$. This defines the boundaries of the $j$ th column of the sub-matrix. If $x_{\text {med }}<X_{j-1}^{\max }$, this angle cannot be used in construction of a diagonally dominant sub-matrix.

6. Continue at Step 4 with the next smallest angle until all measured angles have been processed.

7. For each value of $k$ and $j$, use the value for $\Theta_{k}$ to convert $X_{j}^{\min }$ and $X_{j}^{\max }$ to an energy range $E_{k j}^{\min }-E_{k j}^{\max }$. The sub-matrix element $P_{x}\left(\Theta_{k}, X_{j}\right)$ is the integral of $\mathrm{d} P / \mathrm{d} E$ over this range.

The end result of this process is a number of well-conditioned sub-matrices that can be independently inverted to solve for $F_{\text {coh. }}$. Typical condition numbers for the individual 
sub-matrices are less than 5 . Although the $X_{j}$ points in a particular sub-matrix could be widely spaced, these gaps can be filled in with the results from other sub-matrices.

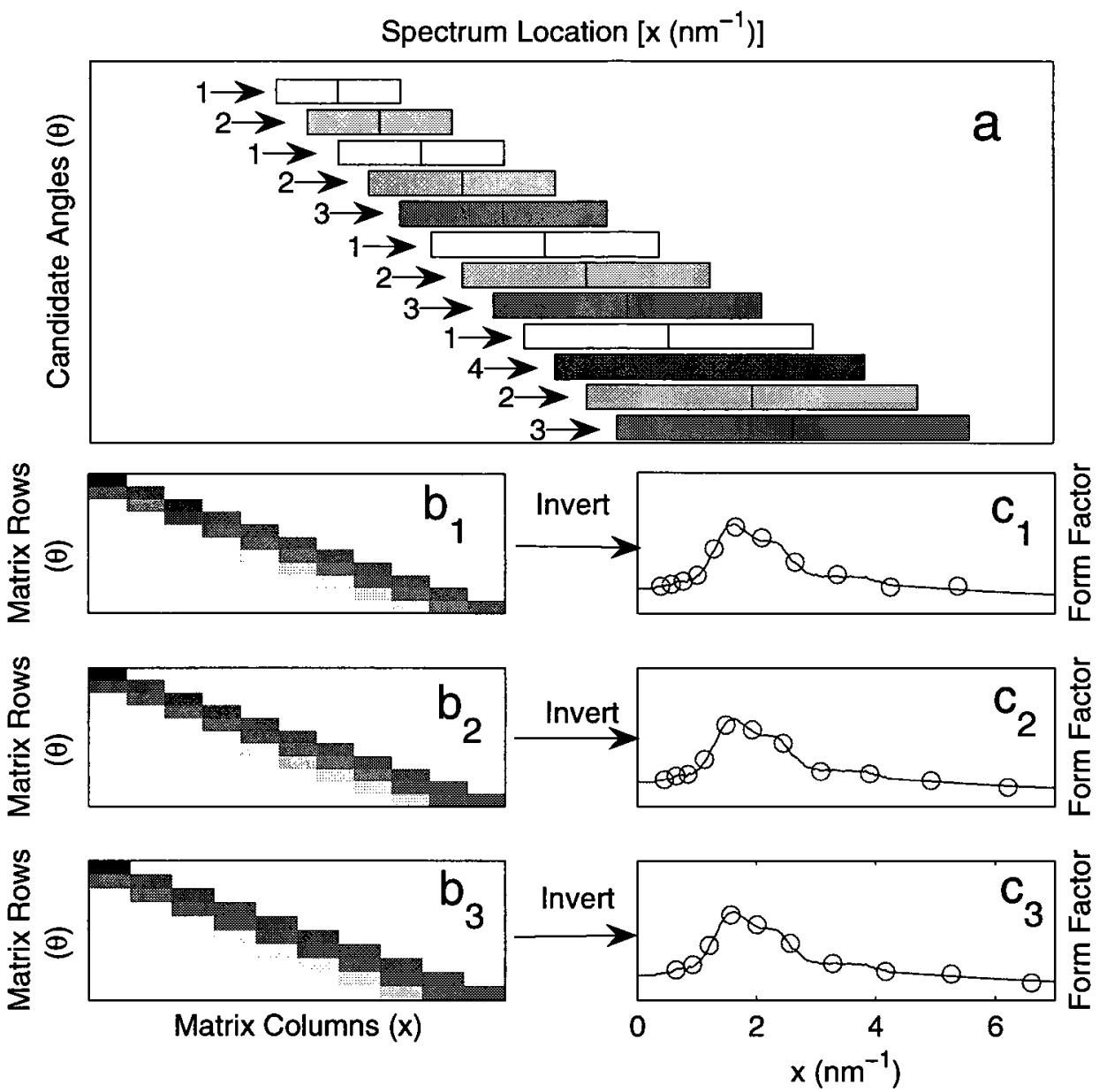

Figure 3.2: Diagrammatic representation of sub-matrix construction. a) shows the extent of the spectrum scaled into $x$ space for each $\theta_{i}$ (for the first few angles only). The line in the middle of each row represents the median energy of the spectrum. The numbers show the proper sub-matrix that the row should be used in. In order to construct diagonally dominant sub-matrices, the midpoint in the row must be past the end-point of the previous row for each sub-matrix. Shading is used to indicate the appropriate groupings. b) shows a visual representation of the first three sub-matrices. Dark values represent matrix elements with large values. Note that each sub-matrix is diagonally dominant. c) shows the result of each sub-matrix inversion (circles) compared to the true value (line). 
An illustrative example is shown in Figure 3.2. This example uses a rectangular spectrum with photons between 30 and $50 \mathrm{keV}$. Because of the symmetry, the median energy of the spectrum is located simply at $40 \mathrm{keV}$.

\subsubsection{Regularization Calculation}

With this method, we attempt to deal with the ill-conditioning of the problem by limiting the possible solutions to those that are physically reasonable. A common characteristic of the numerical solution to an ill-conditioned problem is a large high frequency component, resulting in unrealistic noise which drowns out the actual solution. By imposing a smoothness criterium in the form of Tikhonov regularization [41], the unrealistically noisy solutions are rejected. Although the mathematics involved in determining the optimal smoothness criterium can be quite involved, implementation of the basic Tikhonov regularization is straightforward. In our case, from Equation 3.10:

$$
\underline{\mathbf{P}_{\mathbf{x}}} \mathbf{F}_{\mathbf{c o h}}^{2}=\underline{\chi}^{-1} \mathbf{S}^{\prime}
$$

The ordinary solution would be the generalized inverse:

$$
\mathbf{F}_{\text {coh }}^{\mathbf{2}}=\left(\underline{\mathbf{P}}_{\underline{\mathbf{x}}}^{T} \underline{\mathbf{P}_{\mathbf{x}}}\right)^{-1} \underline{\mathbf{P}}^{T} \underline{\chi}^{-1} \mathbf{S}^{\prime}
$$

Since the matrix $\underline{\mathbf{P}_{\mathbf{x}}}$ is poorly conditioned, Tikhonov regularization calculates an approximate solution to be a slight modification of the generalized inverse:

$$
\mathbf{F}_{\mathbf{c o h}}^{\mathbf{2}}=\left(\underline{\mathbf{P}}_{\underline{\mathbf{x}}}^{T} \underline{\mathbf{P}}_{\mathbf{x}}+\lambda^{2} \underline{\mathbf{I}}\right)^{-1} \underline{\mathbf{P}}_{\underline{\mathbf{x}}}^{T} \underline{\chi}^{-1} \mathbf{S}^{\prime}
$$

where $\lambda$ is a smoothness parameter and $\mathbf{I}$ is the $n \times n$ identity matrix. Larger values of $\lambda$ force smoother solutions to be found. However, the regularization solutions are not strongly dependent on the value of $\lambda$. 


\subsection{Simulation Results}

To verify the validity of these methods of form factor extraction, simulated scatter patterns were generated and the form factors extracted using the functions simsubFFCalc and simregFFCalc. The effective energy results were computed using the function simmonoFFCalc (See Appendix C). All numerical simulations were performed using the assumptions of an ideal photon counting detector $\left[p_{\mathrm{d}}(E)=1, s_{\mathrm{d}}(E)=1\right]$ and negligible target attenuation $\left[\mu_{\mathrm{t}}(E)=0\right]$. We also arbitrarily set the constant factor in Equation 3.3 equal to one. If using experimental data, this constant could be determined by scaling the measured values to the Independent Atom Model (IAM) results as in Johns and Wismayer[23]. The detector plane was located $25 \mathrm{~cm}$ from the target. The signal was measured over an angle range of $\sim 1^{\circ}-25^{\circ}$. We used form factors for water and fat based on Kosanetzky et al[20]. The spectra used for construction of the sub-matrices were taken from Birch, Marshall and Ardran[34] and filtered according to the attenuation coefficient data from Plechaty $e t$ al[5].

\subsubsection{Sub-Matrix Calculations}

An example of the sub-matrix calculation is shown in Figure 3.3. As can be seen, the sub-matrix calculation matches the input form factor quite accurately. The sub-matrix calculation, however, will not work well in all cases. It is strongly dependent on the width of the $\mathrm{d} P / \mathrm{d} E$ function, which is defined by the width of the incident spectrum. In the ideal, monochromatic case, the sub-matrix calculation results in a single, diagonal matrix that is trivially invertible. As the spectrum broadens, the number of sub-matrices required increases and their size decreases. The matrix inversion then becomes less accurate.

Using the function compff_spec (see Appendix C), we investigated the behaviour of the sub-matrix calculation with different types of spectra using artificial triangular spectra for the simulated scattering of water. The function calculates the RMS difference compared 

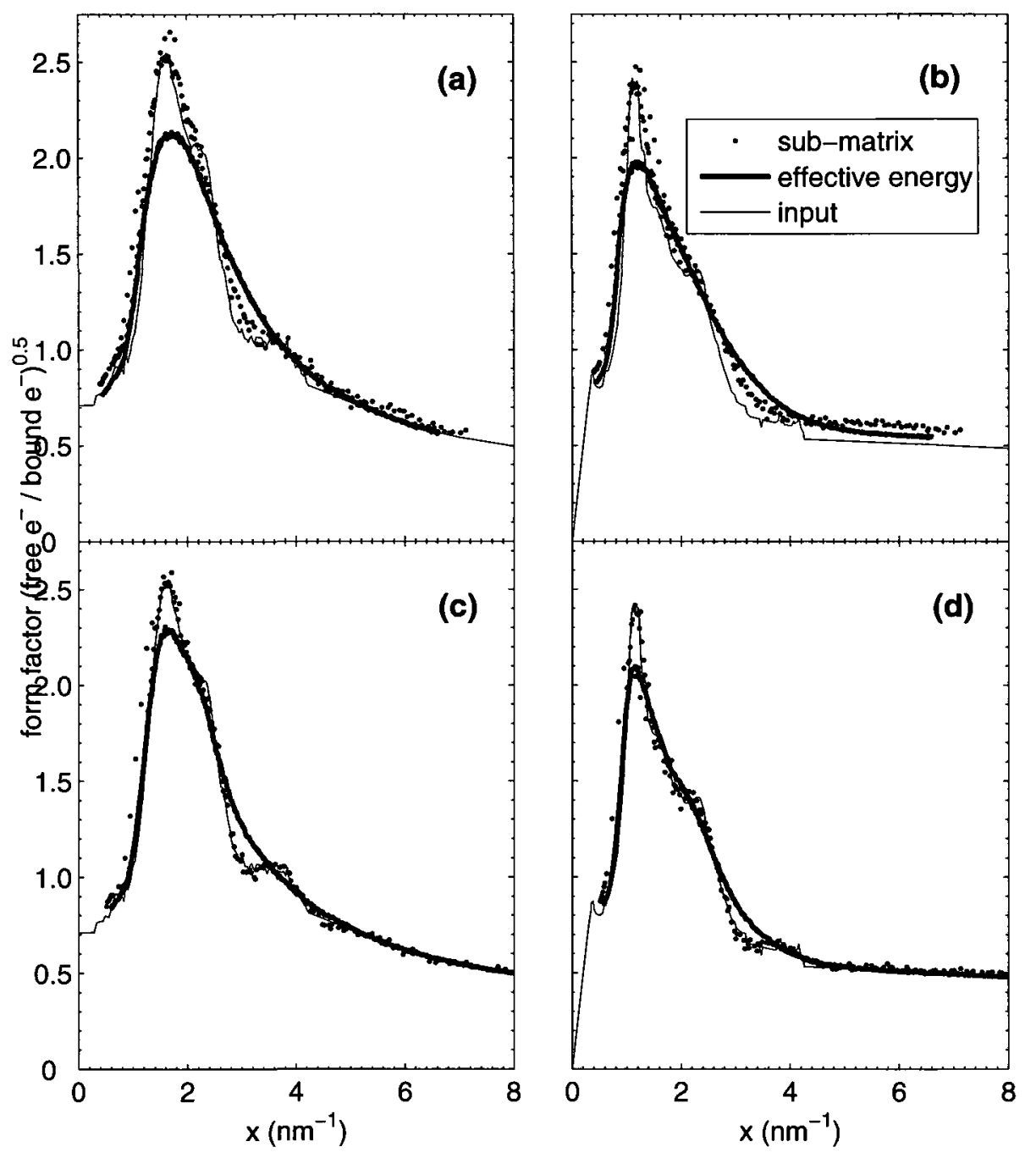

Figure 3.3: Simulated performance of sub-matrix and effective energy calculation of form factors for (a) water with $70 \mathrm{kV}$ spectrum and $0.127 \mathrm{~mm}$ Ho filtration, (b) fat with $70 \mathrm{kV}$ spectrum and $0.127 \mathrm{~mm}$ Ho filtration, (c) water with $90 \mathrm{kV}$ spectrum and $0.2 \mathrm{~mm} \mathrm{~W}$ filtration, (d) fat with $90 \mathrm{kV}$ spectrum and $0.2 \mathrm{~mm} \mathrm{~W}$ filtration. 
to the exact form factor. The results are given in Figure 3.4. The results shown here are for the form factor of water and a specific type of spectrum but qualitatively similar results can be obtained with other inputs.

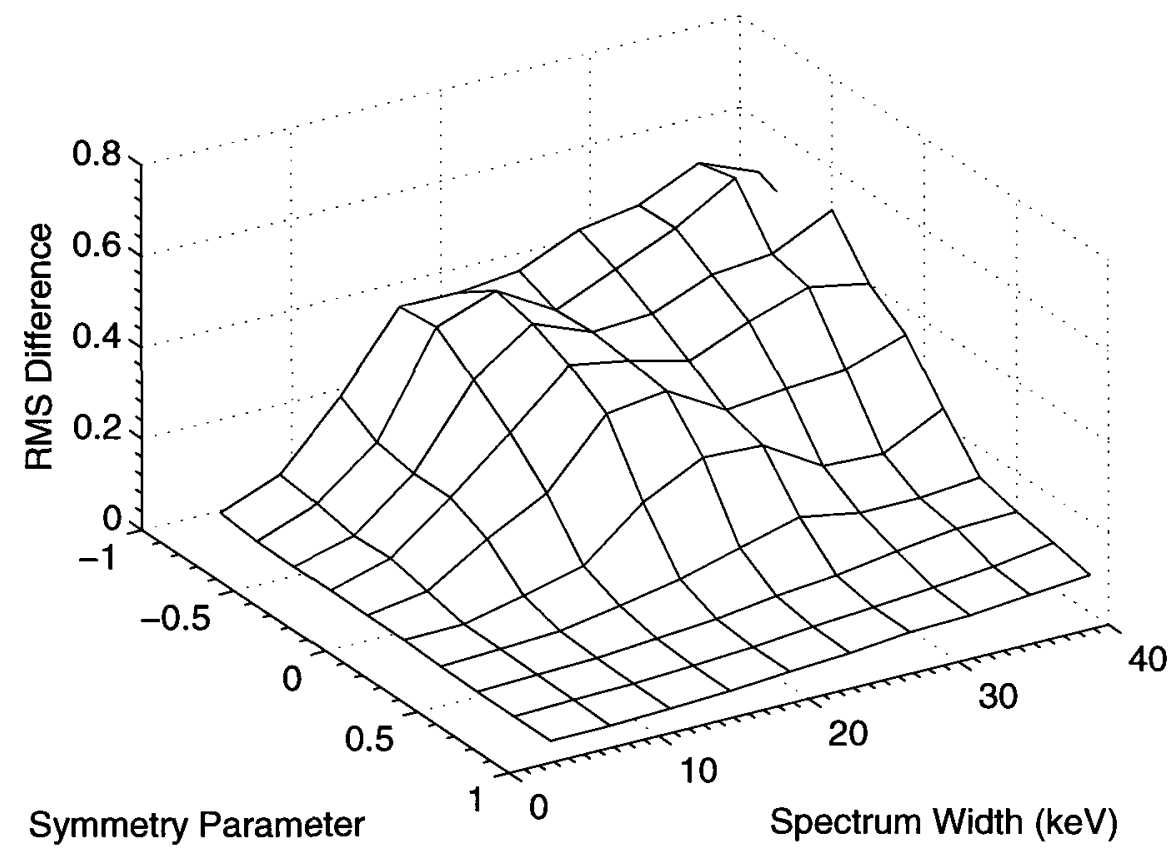

Figure 3.4: Accuracy of the sub-matrix calculation for a range of spectral shapes for simulated scattering from water. The spectra used were triangular with a maximum energy of $60 \mathrm{keV}$. The symmetry parameter determines the location of the peak value. A value of $1(-1)$ corresponds to a peak located at the high (low) energy end of the spectrum.

For very narrow spectra, the sub-matrix calculation reduces to the monochromatic result and gives good agreement with the true result as expected. Figure 3.4 also shows that for wider spectra the agreement remains good if the peak is skewed towards higher energy but rapidly degrades if it is skewed towards lower energies. This is because the submatrix construction process is dependent on the median value of the spectrum. Spectra skewed towards higher energies allow each individual sub-matrix to be larger and hence give more accurate results. This suggests that filtered spectra should improve the accuracy of the calculation.

We also expect the accuracy of the sub-matrix method to degrade as the peaks in the target form factors become sharper. The sub-matrix method is limited to a low resolution 
Table 3.1: Parameters used in constructing artificial form factors

\begin{tabular}{llll}
\hline & Gaussian A & Gaussian B & Constant \\
\hline \hline Peak Location $\left(\mathrm{nm}^{-1}\right)$ & Variable & 3.0 & \\
Standard Deviation $\left(\mathrm{nm}^{-1}\right)$ & Variable & 5.0 & \\
Maximum Value (free $\mathrm{e}^{-}$/bound $\left.\mathrm{e}^{-}\right)^{0.5}$ & 1.6 & 0.4 & 0.5 \\
\hline
\end{tabular}

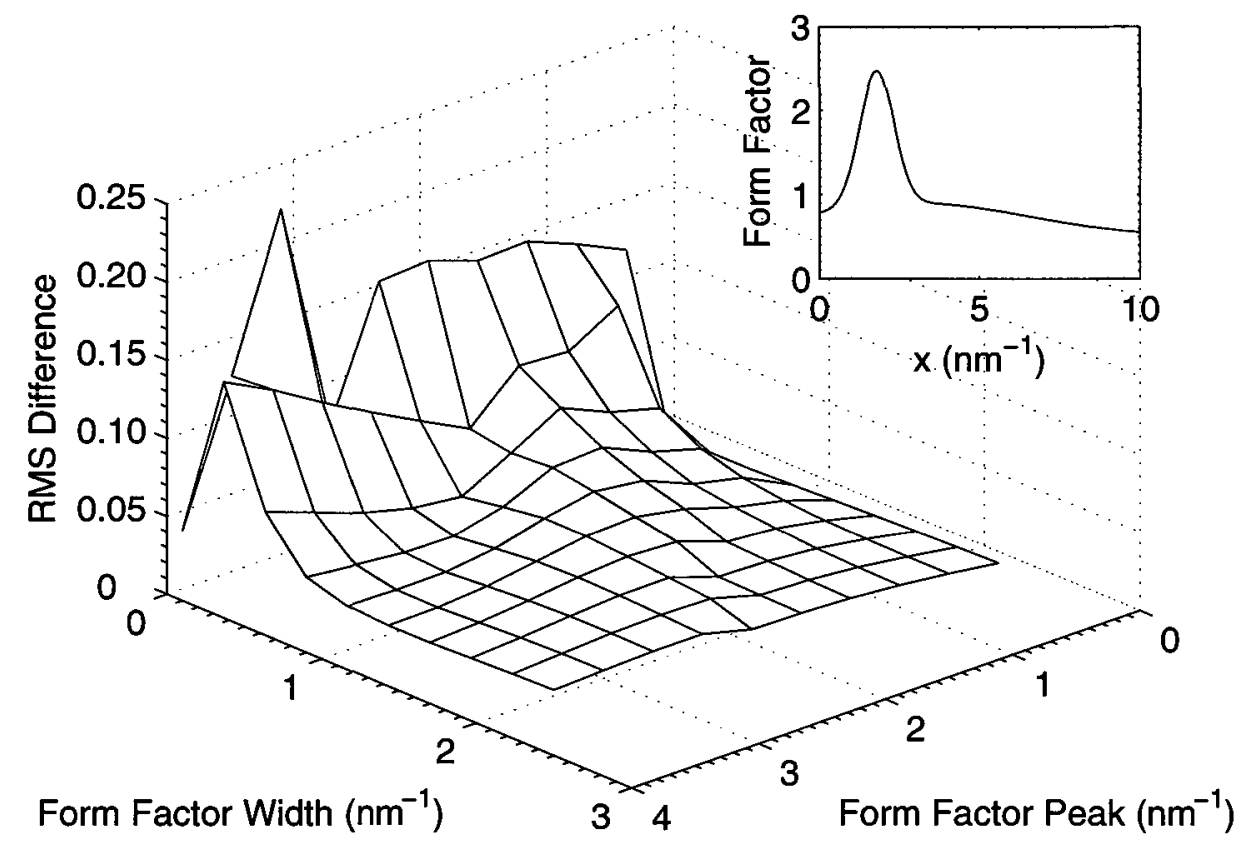

Figure 3.5: Accuracy of the sub-matrix calculation for a range of form factors. The spectrum used was $90 \mathrm{kV}$ with $0.2 \mathrm{~mm} \mathrm{~W}$ filtration in all cases. The inset shows the form factor generated for a peak location of $1.8 \mathrm{~nm}^{-1}$ and peak width of $0.75 \mathrm{~nm}^{-1}$ corresponding roughly to water.

in $x$ because of the relatively large spacing between points for each sub-matrix inversion. Using the function ffcomp_ff (see Appendix C.1), we created artificial form factors by combining Gaussians. This allowed us to smoothly vary the width and peak location of the form factor. Each artificial form factor is composed of two Gaussians and a constant offset in order to match the general shape of typical form factors. The parameters shown in Table 3.1 were chosen to generate form factors qualitatively similar to those of water and fat. Figure 3.5 shows the behaviour of the sub-matrix calculation for a range of these 
artificial form factors. It is clear that as the form factors get narrower, accuracy of the calculation suffers. Accuracy also deteriorates as the peak moves to larger $x$. This is because the individual points in each sub-matrix are more widely spaced at larger $x$ values making it harder to match a sharp peak.

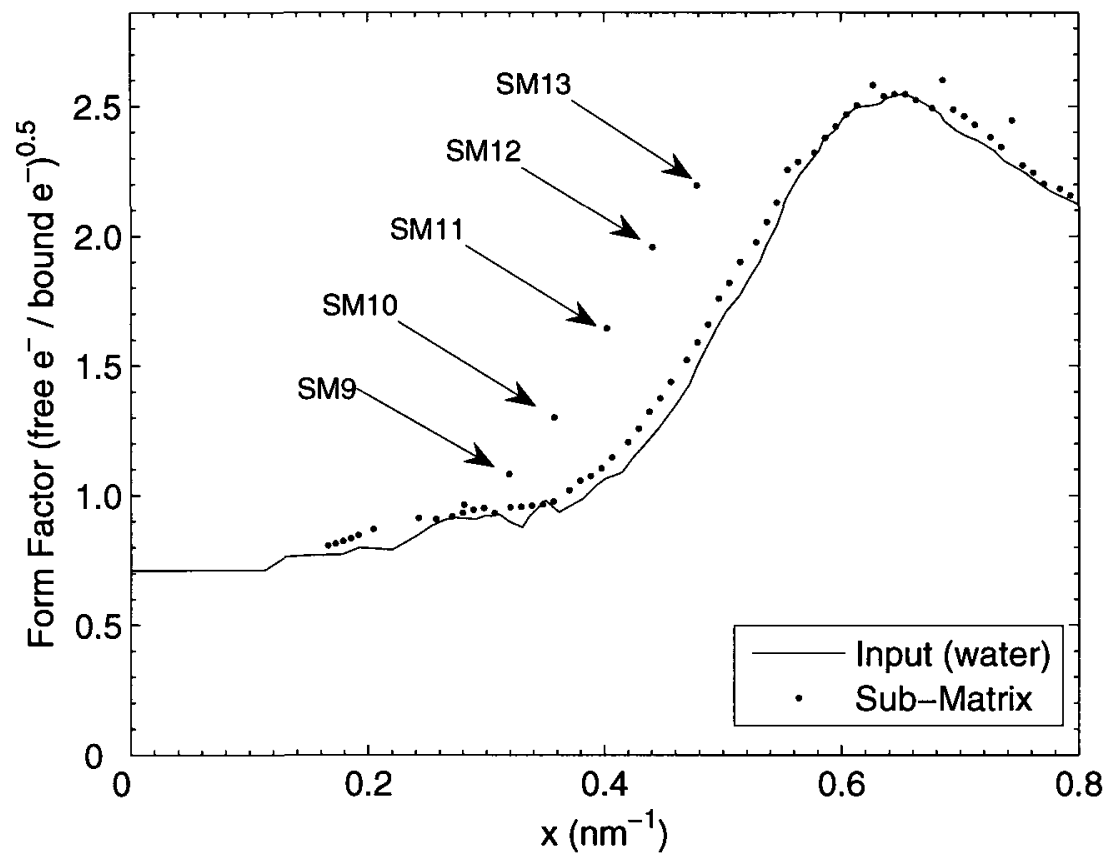

Figure 3.6: The small $x$ region of the form factor determination for water with a $90 \mathrm{kV}$ x-ray spectrum using an additional $0.2 \mathrm{~mm}$ tungsten filtration. The highlighted points show the first data point evaluated using higher order sub-matrices (Sub-matrix 9, $10 \ldots$... . By removing the results from these submatrices, better agreement with the true value can be achieved.

Two improvements to the basic sub-matrix method are necessary to make use of this method in realistic situations. Both involve restricting some of the information that is used in the calculation. The first is sub-matrix truncation. The need for this can be seen in Figure 3.6. In many cases, the higher order sub-matrices prove to be less accurate than the lower order. This is because the first point calculated from any sub-matrix is essentially a monochromatic calculation of the form factor (since the first row of any sub-matrix compresses the entire spectrum down to a single element) and the sub-matrix method relies on this first point being reasonably accurate. At some point, this assumption 
will break down. Thus, by using only the "good" matrices, we can improve the overall agreement of the calculation.

The question of how to determine the location of the cutoff between "good" and "bad" matrices is significant. The method used must not rely on knowledge of the form factor since, in actual experiments, this is not known beforehand. It also must not be overly sensitive to the presence of noise in the system. The method that we have used is based on the slope of the scatter signal as a function of $\theta$. Once the slope becomes larger than a defined cutoff value, we ignore all higher order sub-matrices. The justification for this method is that the slope is an indicator of how much variation is present in the form factor for the range of energies in the spectrum.
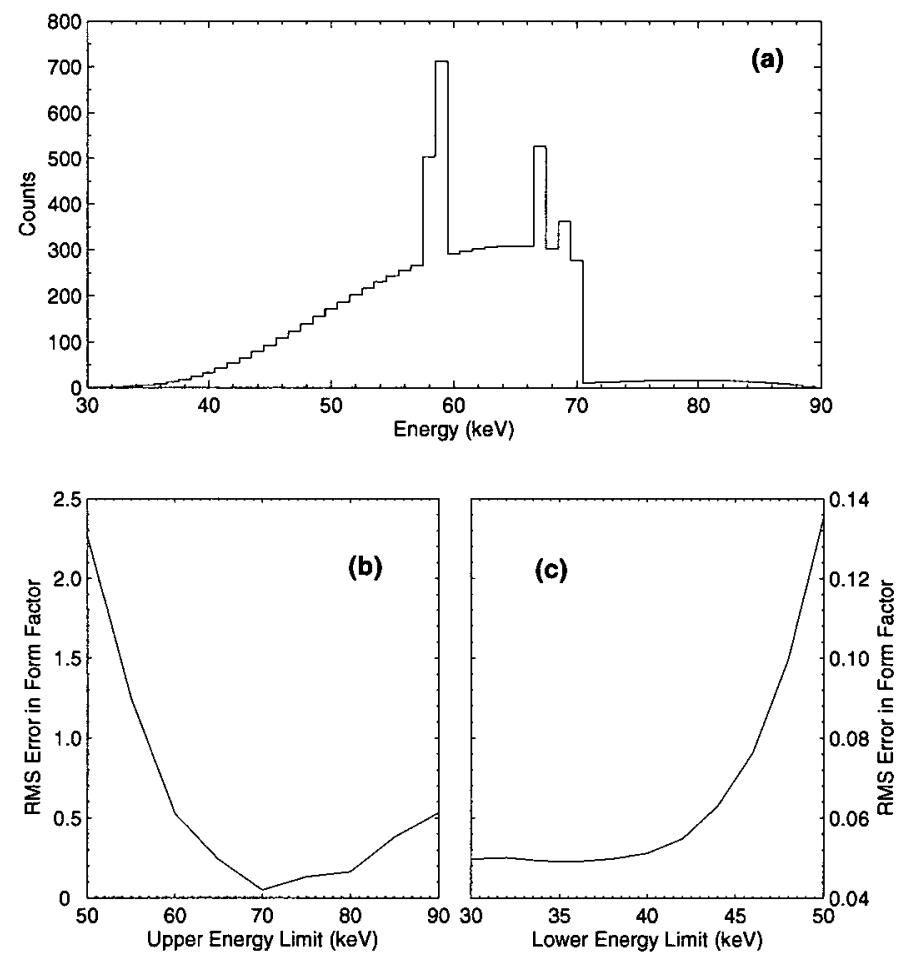

Figure 3.7: The effect of spectrum truncation on the accuracy of the form factor calculation. a) shows the $\mathrm{x}$-ray spectrum for a $90 \mathrm{kV} \mathrm{W}$ anode tube with $0.2 \mathrm{~mm}$ additional $\mathrm{W}$ filtration. The K-edge is clearly visible at 70 $\mathrm{keV}$. b) shows the effect of restricting the upper edge of the spectrum on calculation accuracy while c) shows the effect of restricting the lower edge of the spectrum. In c), the upper limit was set to the optimal location of 70 $\mathrm{keV}$. Note the difference in scale between $\mathbf{b}$ ) and $\mathbf{c}$ ). 
The second method involves artificially restricting the width of the spectrum used in the calculation. As seen in Figure 3.4, the sub-matrix calculation degrades as the spectral width increases. Thus, using a narrower spectrum will improve the calculation. In typical $\mathrm{x}$-ray spectra, the bulk of the spectrum is contained in a central energy region. By truncating the relatively small number of x-ray photons at the extremes of the spectrum, the width can be significantly reduced resulting in a more accurate sub-matrix calculation. This is most effective by using the sharp cutoff present in a K-edge filtered spectrum. By cutting off the spectrum above the K-edge, significant improvements in accuracy can be obtained. The more gradual change at the lower energy end of the spectrum is much less sensitive to the cutoff energy. This can be seen in Figure 3.7.

\subsubsection{Regularization Calculations}

The regularization calculation is implemented in the function simregFFCalc (see Appendix C.1). The effect of varying $\lambda$ is shown in Figure 3.8. The progression in smoothness with increasing values of $\lambda$ is clear. We used $\lambda=0.1$ for the remaining calculations in this section and in Chapter 4 as a tradeoff between noise and resolution for the types of form factors expected for tissue measurements. The $\underline{\mathbf{P}_{\mathbf{x}}}$ matrix was constructed to be overdetermined by a factor of four.

The functions compff_spec and compff_ff were used to verify the regularization calculations in the same manner as was done for the sub-matrix calculations. The results of varying the spectral shape and the shape of the form factor are shown in Figures 3.9 and 3.10. The regularization calculations appear to follow the same general trends as the sub-matrix calculation for varying spectral shapes, working best for narrow spectra, skewed toward high energies. It seems less sensitive to the spectral characteristics than the sub-matrix method. On the other hand, the regularization calculations show the most consistent results for form factors peaked at larger $x$ values, which is very different behaviour as compared to the sub-matrix calculation. 


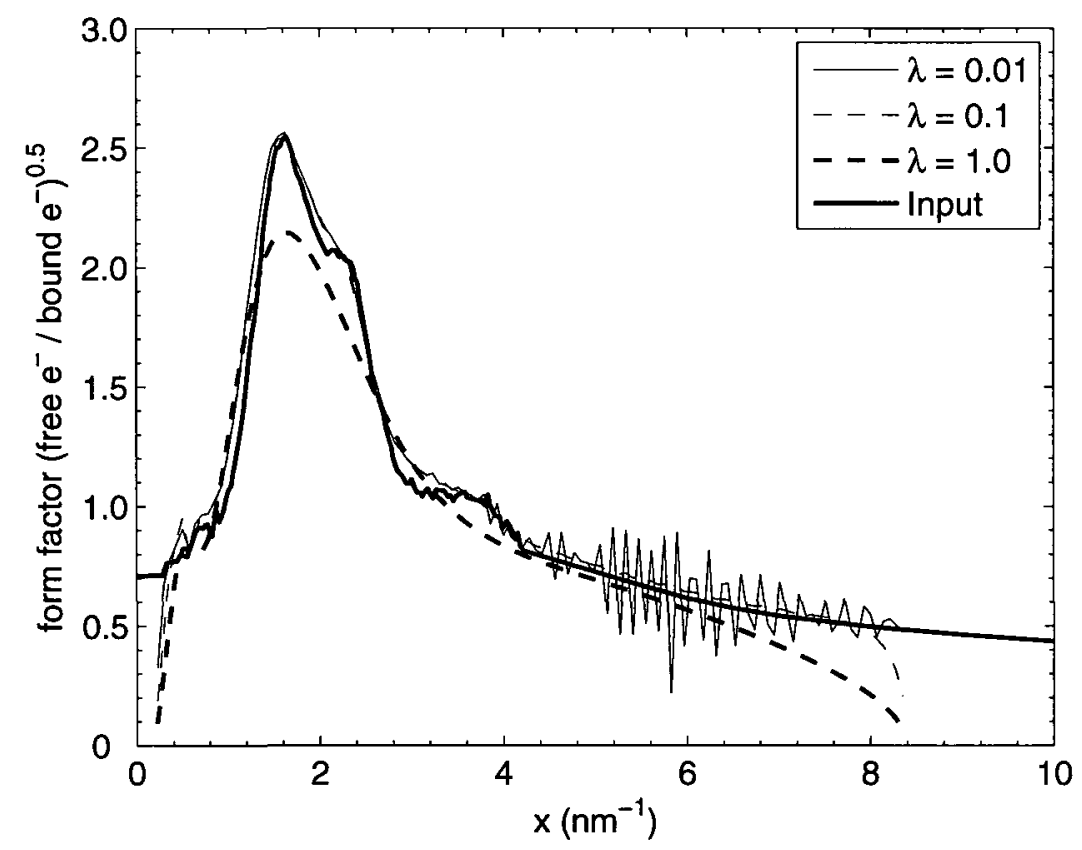

Figure 3.8: Comparison of the regularization calculation with varying smoothness parameters. The calculation was done using a $70 \mathrm{kV}$ spectrum with $0.127 \mathrm{~mm}$ of Ho filtration and $0.1 \mathrm{~mm} \mathrm{Al}$ filtration.

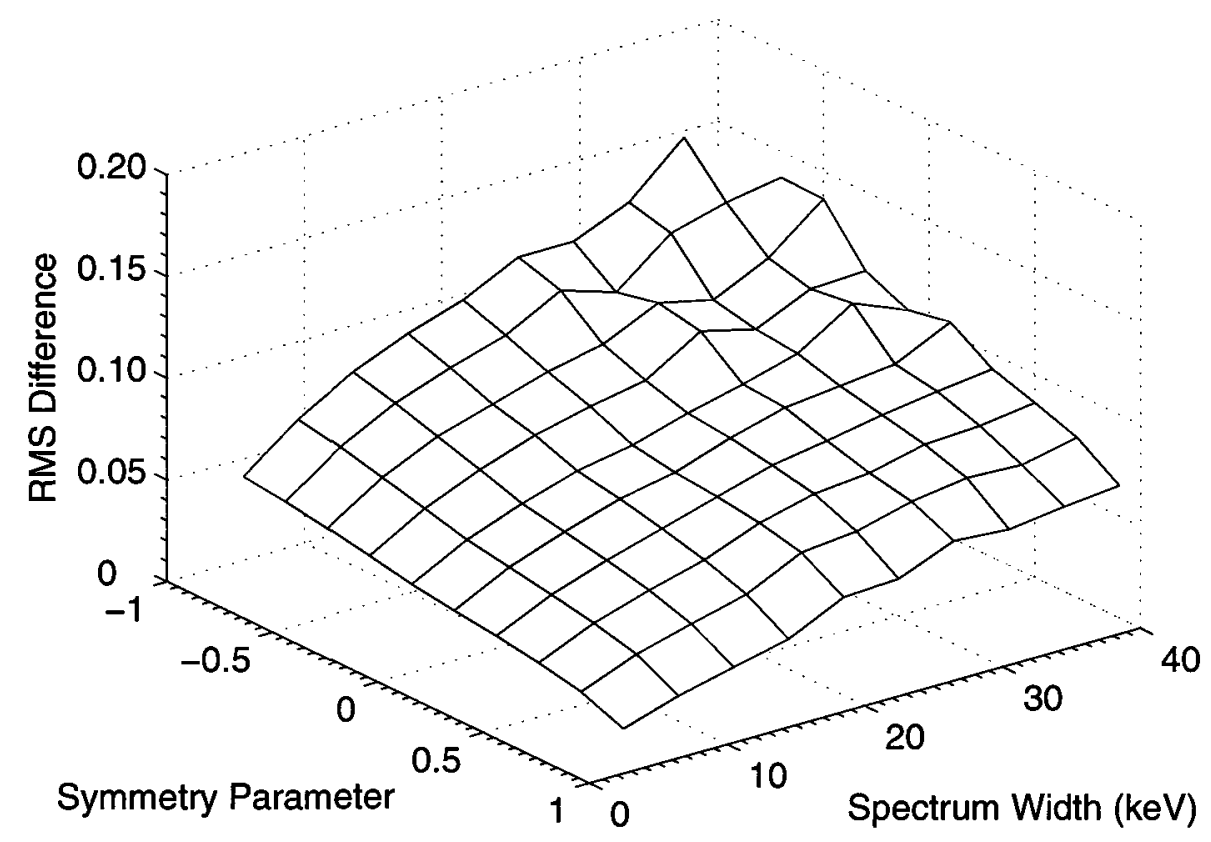

Figure 3.9: Accuracy of the regularization calculation with $\lambda=0.1$ and a range of spectral shapes. The spectra used were triangular with a maximum energy of $60 \mathrm{keV}$. The symmetry parameter determines the location of the peak value. A value of $1(-1)$ corresponds to a peak located at the high (low) end of the spectrum. 


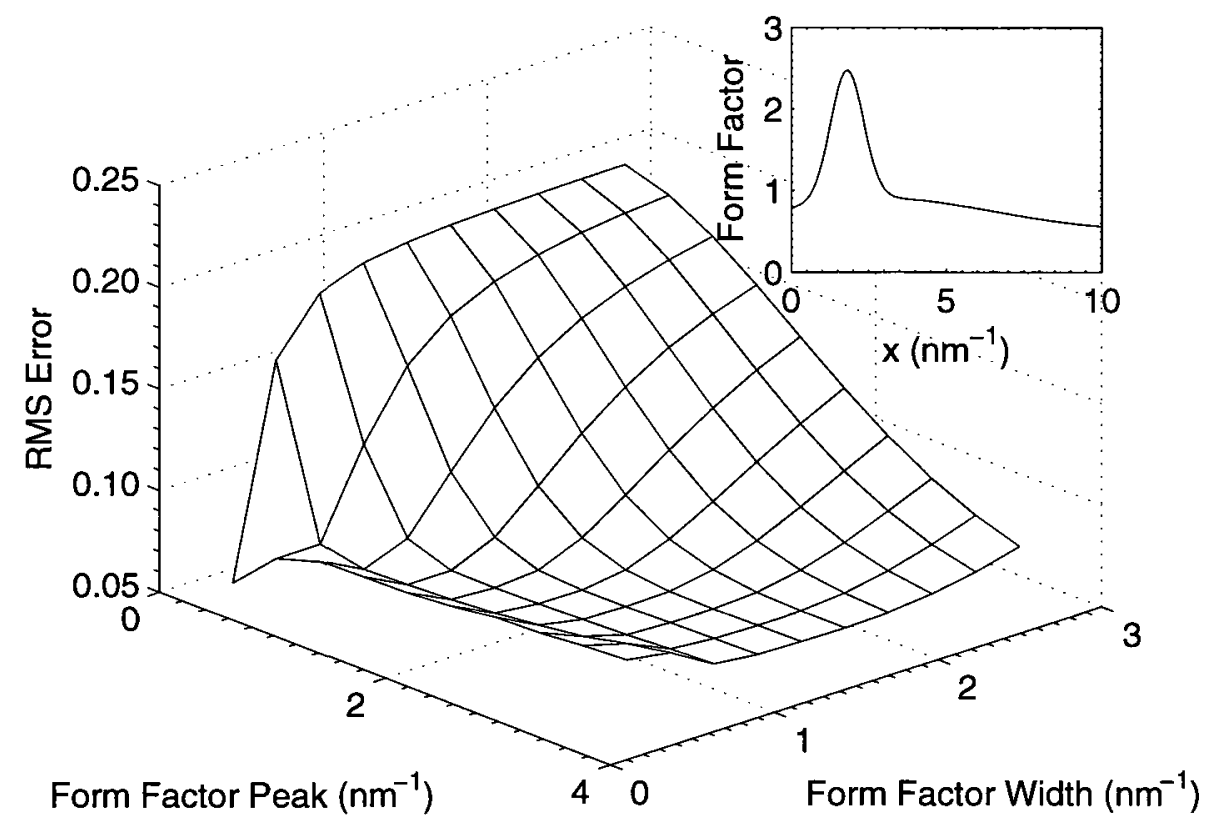

Figure 3.10: Accuracy of the regularization calculation with $\lambda=0.1$ and a range of artificial form factors. The spectrum used was $90 \mathrm{kV}$ with 0.2 $\mathrm{mm} \mathrm{W}$ filtration in all cases. The inset shows the form factor generated for a peak location of $1.8 \mathrm{~nm}^{-1}$ and peak width of $0.75 \mathrm{~nm}^{-1}$ corresponding roughly to water.

\subsection{Discussion}

Both the sub-matrix and regularization methods show improvements in matching the peak characteristics over the effective energy calculation but both have limitations as well. Both methods are approximations. Even with perfect knowledge of the scattering signal, they do not give exact results. The sub-matrix method is only valid within a certain range of parameters. Outside of this range, accuracy deteriorates rapidly.

The regularization method seems to work in a wider set of circumstances than the sub-matrix method but requires additional knowledge of the expected form factor, in the choice of the smoothness parameter, $\lambda$.

These simulations have shown some encouraging results, as well as outlining the regions of validity of the two types of calculations. The simulations are limited in several ways, though. First, all scattering is assumed to take place at the centre of the target. Sec- 
ond, image blurring in the detector is ignored. In order to test the angle-dispersive form factor measurement more thoroughly, experimental measurements were made. These will be investigated in the next chapter. 


\section{CHAPTER 4}

\section{ANGLE-DISPERSIVE FORM FACTOR}

\section{MEASUREMENTS USING IMAGING}

\section{DETECTORS - RESULTS}

In order to verify the results of the sub-matrix and regularization simulations presented in Chapter 3, I measured the scattering signal of water and fat samples by recording diffraction images with a storage phosphor plate. I used different $\mathrm{x}$-ray tube voltages and compared the measured form factors to those measured with standard angle-dispersive diffractometers. The results of these measurements are shown below.

\subsection{Experimental Details}

A rotating $\mathrm{W}$-anode $\mathrm{x}$-ray tube with a Machlett Dynamax 69M insert was used to provide a series of $\mathrm{x}$-ray spectra. The $\mathrm{x}$-ray tube was powered by a single phase generator (Picker GX550). The detector was a $400 \mu \mathrm{m}$ thick Kodak $20 \mathrm{~cm}$ x $25 \mathrm{~cm} \mathrm{BaFBr}$ storage phosphor plate with cassette and the images were read by a Bio-Rad Personal Molecular Imager (Bio-Rad, Hercules, CA) using the included "Quantity One" software with $0.1 \mathrm{~mm}$ pixels.

All measurements were made with the $1.2 \mathrm{~mm}$ nominal focal spot and $60 \mathrm{~Hz}$ anode rotation speed. Samples were placed in a cylindrical plastic petri dish which was then 


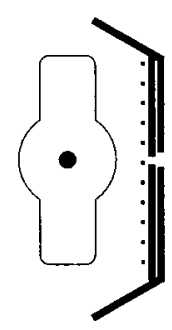

A B

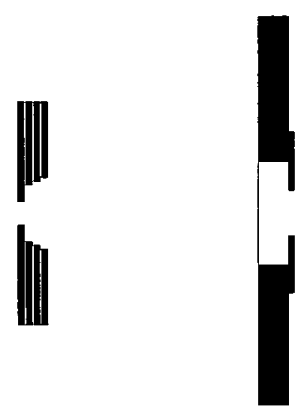

D

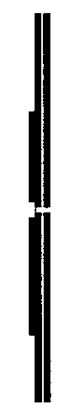

$\mathrm{E}$

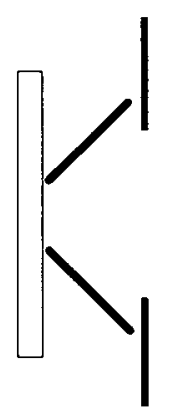

F G H

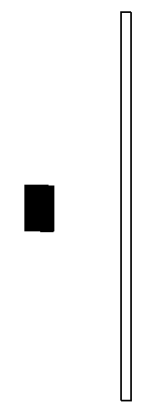

I J

Figure 4.1: Experimental layout. Any filters used in the exposures were located at collimator B on the dotted line. Item $G$ is a truncated square pyramid with 10 and $25 \mathrm{~mm}$ openings. All other apertures are circular. See Table 4.1 for the dimensions of each item.

Table 4.1: Collimation dimensions. Multiple entries under the thickness and diameter headings represent multiple layers of collimation, in order from left to right in Figure 4.1.

\begin{tabular}{lllll}
\hline Label & Description & $\begin{array}{l}\text { Distance from source } \\
\text { to centre }(\mathrm{mm})\end{array}$ & $\begin{array}{l}\text { Thickness } \\
(\mathrm{mm})\end{array}$ & $\begin{array}{l}\text { Diameter } \\
(\mathrm{mm})\end{array}$ \\
\hline \hline A & Source & 0 & & \\
$\mathrm{~B}$ & Pb Collimation & 85 & 2,2 & 1,2 \\
$\mathrm{C}$ & Pb Collimation & 200 & $2,2,2,2$ & $3.0,7.5,8.5,9.5$ \\
$\mathrm{D}$ & Pb Collimation & 240 & $5.5,2$ & 40,10 \\
$\mathrm{E}$ & Pb Collimation & 305 & $2,3,3$ & $2,1,1$ \\
$\mathrm{~F}$ & Scattering Target & 380 & 9 & 52 \\
$\mathrm{G}$ & Pb Collimation & 397 & & \\
$\mathrm{H}$ & Pb Collimation & 410 & 2.5 & 25 \\
I & Pb Beam stop & 595 & 5 & 8.5 \\
J & Screen detector & 630 & & \\
\hline
\end{tabular}


sealed with epoxy and placed vertically in the beam path. Background exposures with empty petri dishes were performed after each target exposure.

A series of $\mathrm{Pb}$ collimation apertures was arranged in the beam path to confine the beam as closely as possible to the ideal pencil beam configuration and also to ensure that only $\mathrm{x}$ rays scattered in the target were able to reach the detector. See Figure 4.1 and Table 4.1 for details. All apertures were mounted on an optical bench to ensure stability.

Table 4.2: Exposure parameters used. Radiographic mode uses a large tube current (200 mA) for a short exposure while fluoroscopic mode uses a small tube current ( $2 \mathrm{~mA}$ ) for a long exposure. Radiographic mode was the only option available for the $50 \mathrm{kV}$ exposure. The tube has an inherent filtration of $0.5 \mathrm{~mm} \mathrm{Al}$.

\begin{tabular}{llll}
\hline Potential $(\mathrm{kV})$ & Mode & Exposure $(\mathrm{mAs})$ & Additional Filtration \\
\hline \hline 50 & Radiographic & 6000 & $1.0 \mathrm{~mm} \mathrm{Al}$ \\
70 & Fluoroscopic & 2400 & $0.127 \mathrm{~mm} \mathrm{Ho}+1.0 \mathrm{~mm} \mathrm{Al}$ \\
92 & Fluoroscopic & 6000 & $0.2 \mathrm{~mm} \mathrm{~W}$ \\
\hline
\end{tabular}

Measurements were made of water at three tube potentials and filtrations as shown in Table 4.2. These measurements were repeated for the fat sample with 50 and $70 \mathrm{kV}$ potentials only. The data consist of a number of circularly symmetric scatter patterns as shown in Figure 4.2. The images were analyzed using the program ImageDisp (see Appendix C). We calculated the average values in a series of $0.2 \mathrm{~mm}$ wide annular rings about the centre of each scatter pattern. Combined with the knowledge of the target to detector distance $d_{\mathrm{t}}$ from Table 4.1 , this yields the scatter intensity $S$ as a function of scattering angle $\theta$. To ensure consistency and repeatability, all scatter measurements were repeated between three and seven times and the average value was used in all calculations.

The repeated exposures showed some fairly large differences from one dataset to the next, as shown in Figure 4.3. However, the data varied only by an overall scaling constant suggesting that the overall photon fluence varies from one exposure to the next but that 

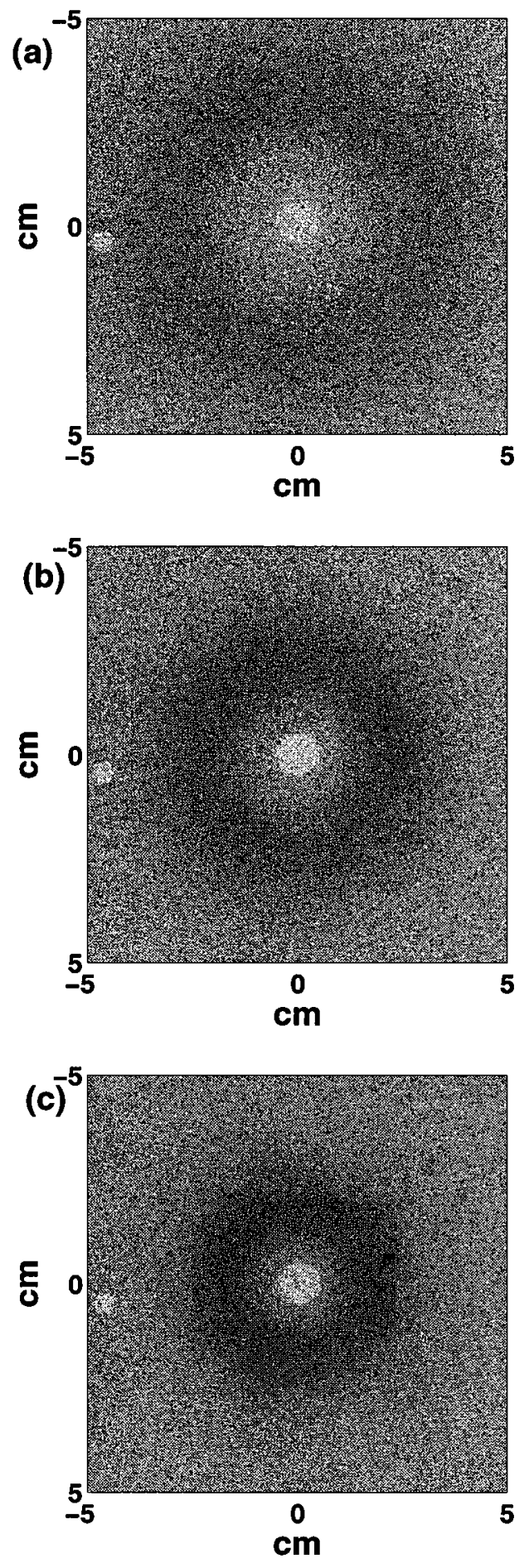

Figure 4.2: Sample scatter patterns from a water target measured with a) $50 \mathrm{kVp}$, b) $70 \mathrm{kVp}$ and c) $92 \mathrm{kVp}$ spectra. Note the circular symmetry. The circular spot on the left of the images comes from a small $\mathrm{Pb}$ disc placed on the storage plate for positioning purposes. 


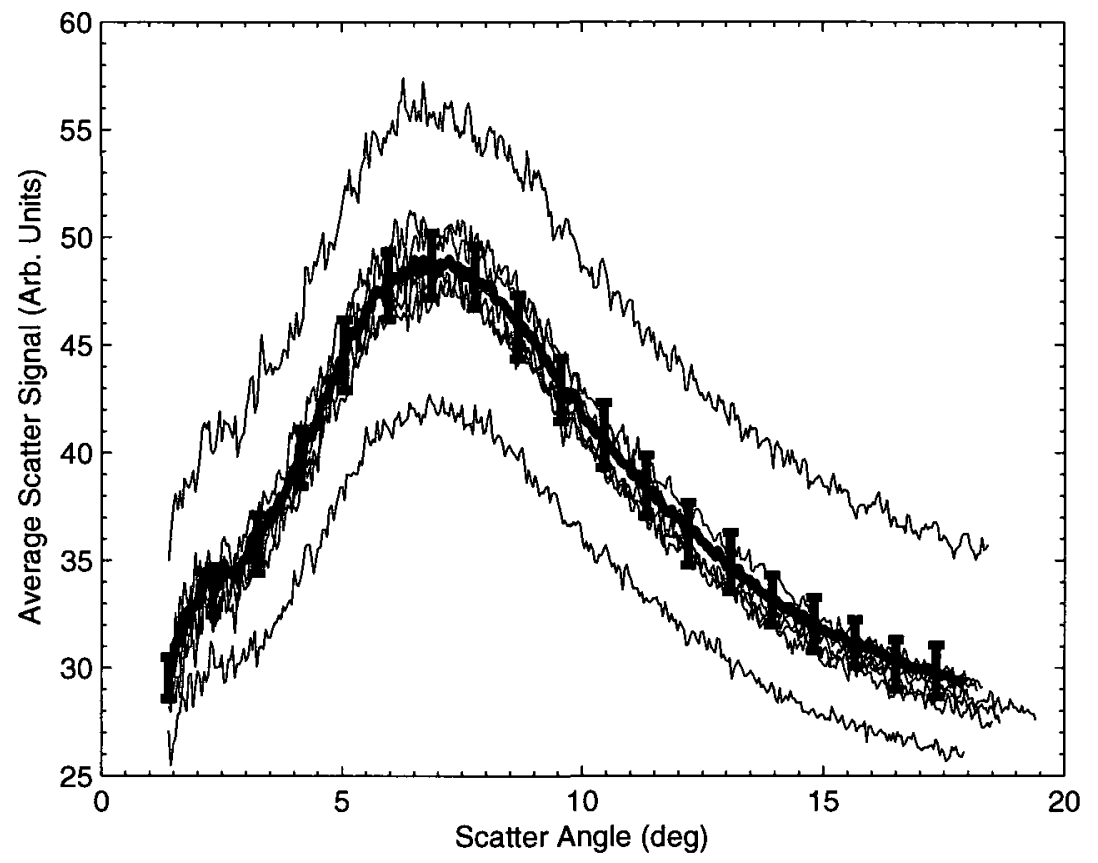

Figure 4.3: Seven repeated measurements of the scatter pattern of water using a $50 \mathrm{kVp}$ spectrum. The dark line shows the average value. Error bars represent the standard deviation of the mean and are only shown for every 20th point for clarity.

the spectrum shape remains constant. An attempt at monitoring the tube output was made using an ionization chamber, located in the path of the beam between collimators $\mathrm{D}$ and $\mathrm{E}$ in Figure 4.1, but the scatter signal did not correlate well with the ionization chamber measurements. This could have been due to the very tight, narrow-beam collimation.

The same procedure was performed for the background exposures at all three potentials. The backgrounds were subtracted from the combined signals using the function sub_diffbg (see Appendix C.1). The background subtracted scatter signals measured with water and fat are shown in Figure 4.4. The errors in these measurements are dominated by the interexposure variations in the scatter patterns. 


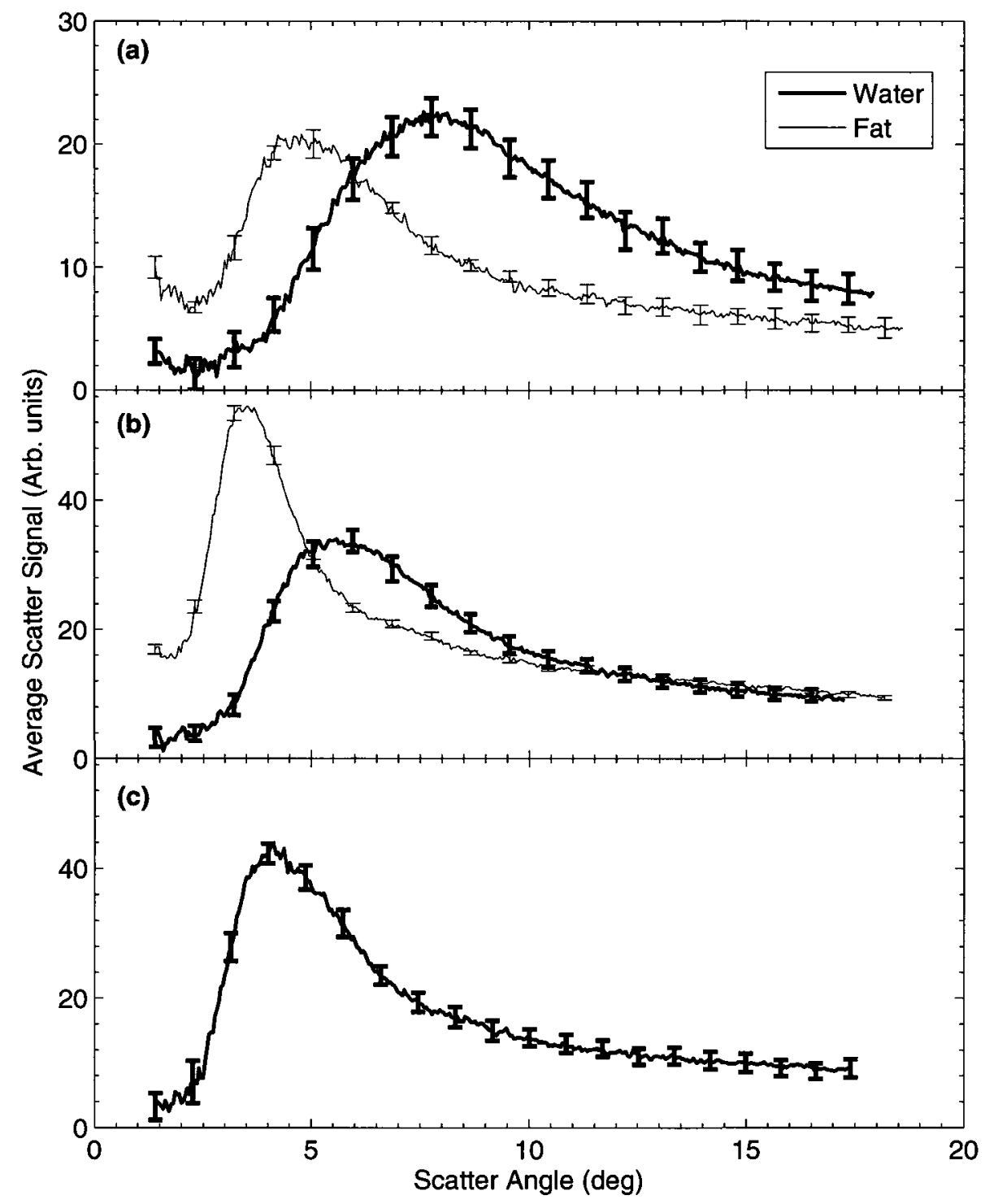

Figure 4.4: Average scatter signal of water and fat measured in our experiment. (a), (b) and (c) represent 50,70 and $92 \mathrm{kV}$ respectively. The fat sample was not measured at $92 \mathrm{kV}$. Error bars are only shown for every twentieth point for clarity. 


\subsection{Measuring $\mathrm{d} P / \mathrm{d} E$}

In order to extract the form factors from the measured data, the $\mathrm{d} P / \mathrm{d} E$ function must be known from Equation 3.5. We used $\mathrm{d} \Phi / \mathrm{d} E$ spectra measured with our High Purity Germanium (HPGe) spectrometer whenever possible. Unfortunately, the fluence rate in radiographic mode was too high for the spectrometer to measure so we were forced to rely on catalogue spectra [34] for the $50 \mathrm{kV}$ exposures. Attenuation coefficients for the filters and targets were applied where necessary based on tabulated attenuation coefficients [5]. The spectra used are shown in Figure 4.5.

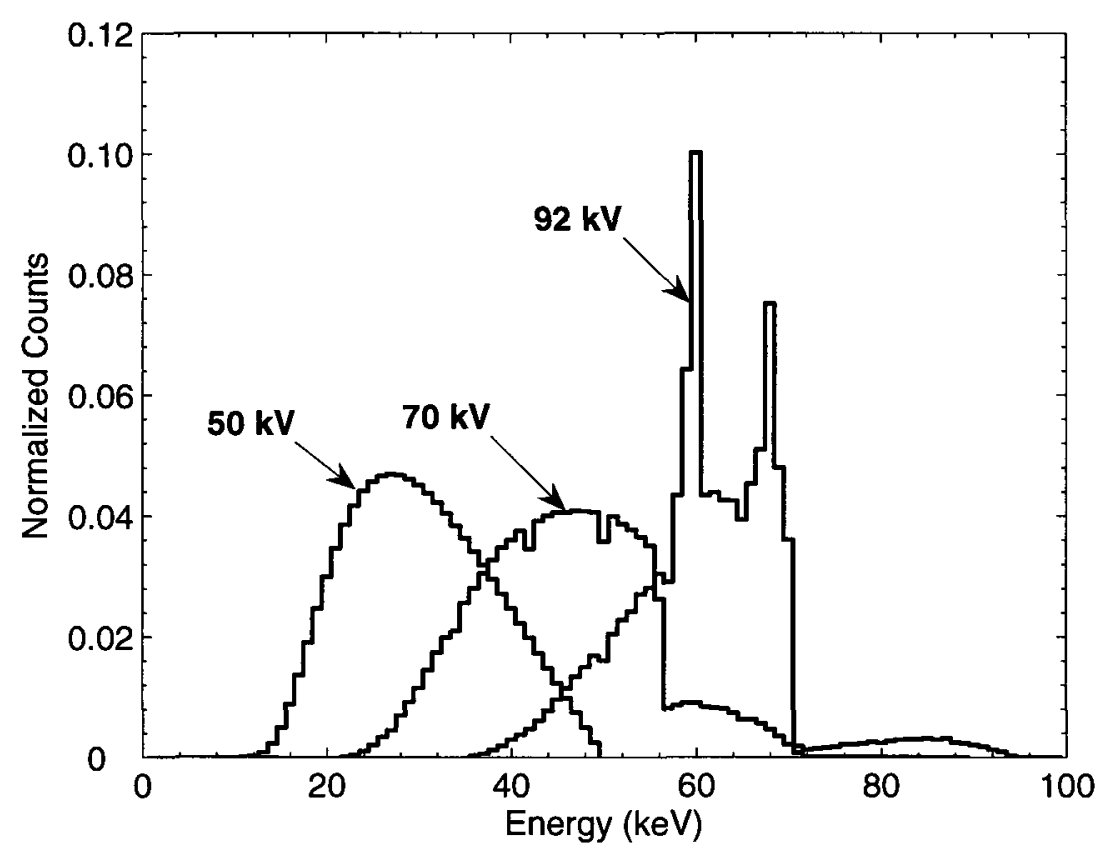

Figure 4.5: Spectra used in angle-dispersive measurements. The $50 \mathrm{kV}$ spectrum was taken from published tables [34] and numerically filtered with attenuation coefficient data [5]. The filtration used with each spectrum can be found in Table 4.2. The 70 and $92 \mathrm{kV}$ spectra were measured in our lab using a HPGe detector.

The functions $p_{\mathrm{d}}(E)$ and $s_{\mathrm{d}}(E)$ in Equation 3.5 are dependent on the detector type. As discussed in Chapter 2, we treated the storage phosphor plate as an ideal energy integrating detector with $s_{\mathrm{d}}(E)=E$ in our calculations. The probability of a photon interacting with 


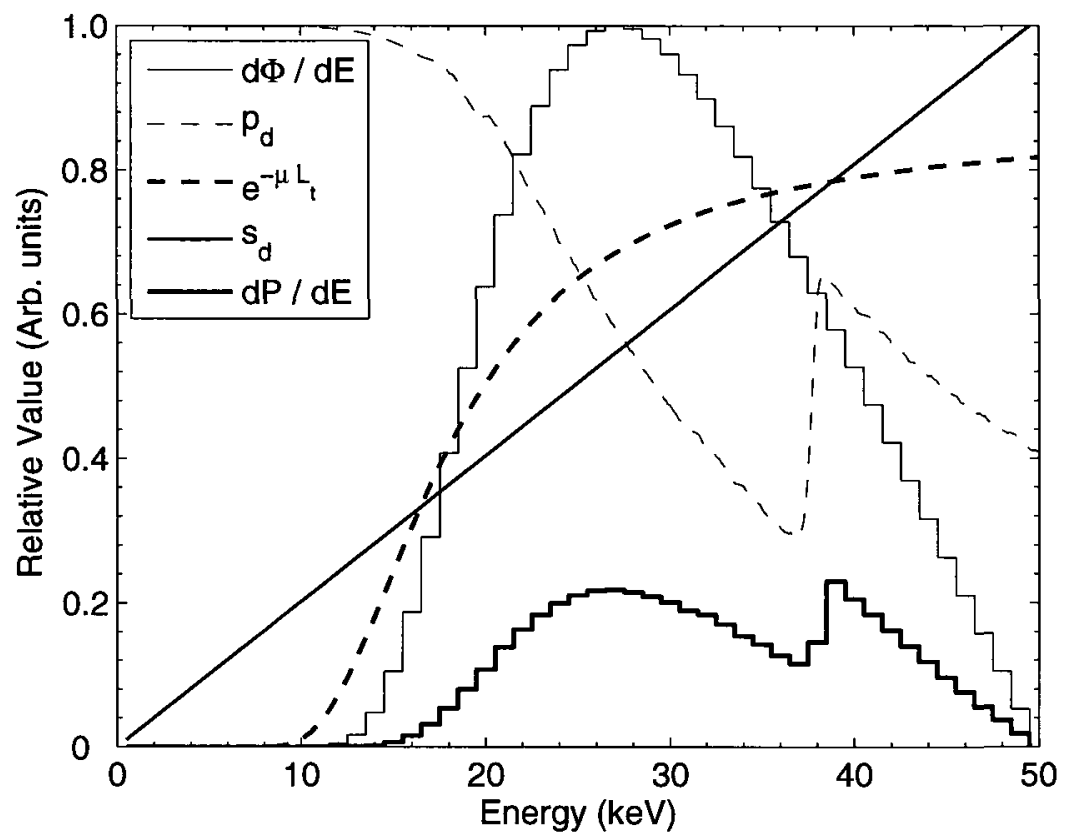

Figure 4.6: Calculated $\mathrm{d} P / \mathrm{d} E$ function for $50 \mathrm{kV}$ beam showing the contribution from each factor in Equation 3.5. The target is assumed to be water.

the detector, $p_{\mathrm{d}}(E)$, is dependent on the quantum efficiency of the detector. We used our HPGe detector to measure the quantum efficiency of the image plate by taking the binby-bin ratio of spectra measured with and without the plate in the path of the beam. The different contributions to $\mathrm{d} P / \mathrm{d} E$ are shown in Figure 4.6.

Table 4.3: Spectrum truncation used with each input $\mathrm{d} \Phi / \mathrm{d} E$ spectrum for the sub-matrix method.

\begin{tabular}{llll}
\hline $\begin{array}{l}\text { Potential } \\
(\mathrm{kV})\end{array}$ & $\begin{array}{l}\text { Minimum Energy } \\
(\mathrm{keV})\end{array}$ & $\begin{array}{l}\text { Maximum Energy } \\
(\mathrm{keV})\end{array}$ & $\begin{array}{l}\text { Counts excluded } \\
(\%)\end{array}$ \\
\hline \hline 50 & 15 & 45 & 3 \\
70 & 25 & 55 & 13 \\
92 & 35 & 70 & 4 \\
\hline
\end{tabular}

As discussed in Section 3.3.1, the sub-matrix method works better if the region of the spectrum above the K-edge of the filter is artificially removed from the calculation. The 
truncation that was used with each spectrum in our experiment is given in Table 4.3.

\subsection{Form Factor Measurements}

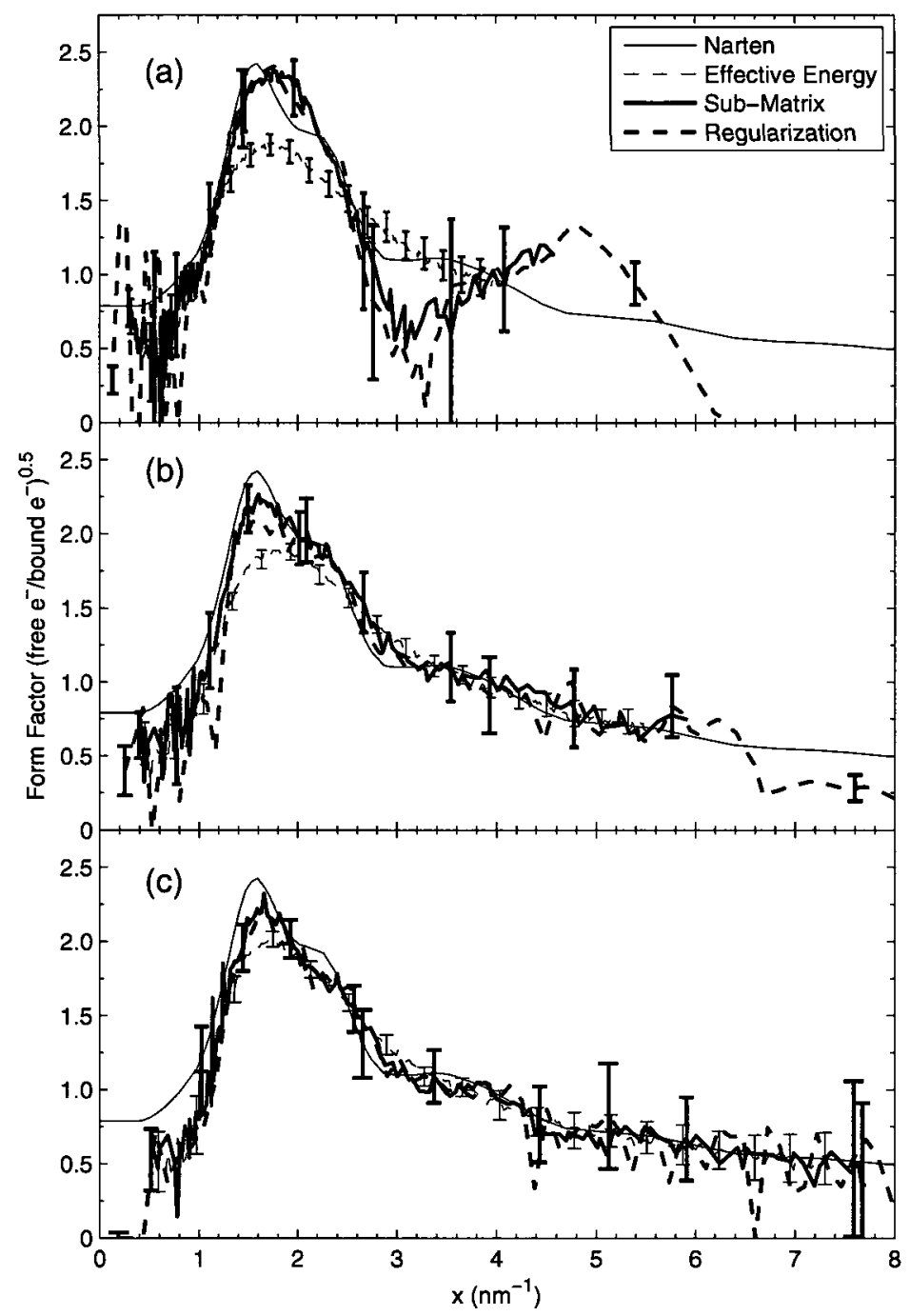

Figure 4.7: Form factors for water extracted from measurements at (a) $\mathbf{5 0}$ $\mathrm{kV}$, (b) $70 \mathrm{kV}$, (c) $92 \mathrm{kV}$. Error bars are shown for only every twentieth point for clarity.

The form factor for water was calculated from our measured data of Figure 4.4 by the methods described in Chapter 3. Results were calculated using the functions expmonoFFCalc, expsubFFCalc, and expregFFCalc in Appendix C.1. The results are shown in Figure 4.7 compared to previous measurements [19]. The most significant difference between the 
effective energy method and the two new methods is seen at $50 \mathrm{kV}$. The sub-matrix and regularization methods match the known water peak better than the effective energy calculation but are noisier and less reliable at larger $x$ values. Both methods show what appears to be oscillatory behaviour. We speculate that this was a result of the lower signal strength with the $50 \mathrm{kV}$ exposure. Oscillatory behaviour is quite common in solutions of ill-conditioned problems. The 70 and $92 \mathrm{kV}$ exposures show some improvement over the effective energy calculation in the peak area and generally good agreement at larger $x$ values. All of our measurements show a slightly smaller peak value than Narten's data.

The calculated values are compared quantitatively to reference data $\left(F_{\text {ref }}\right)$ in Table 4.4 by computing the average absolute relative difference:

$$
D_{\mathrm{av}}=\frac{1}{N} \sum_{i=1}^{N}\left|\frac{F_{\mathrm{coh}}\left(x_{i}\right)}{F_{\mathrm{ref}}\left(x_{i}\right)}-1\right|
$$

where $N$ is the number of points that were measured.

Table 4.4: Average absolute relative difference $D_{\text {av }}$ over the range 0.5 to $4.0 \mathrm{~nm}^{-1}$ of each calculated form factor for water shown in Figure 4.7 as compared to Narten's data [19]. All measured form factors were smoothed by 5 point rectangular averaging.

\begin{tabular}{llll}
\hline Tube Potential $(\mathrm{kV})$ & Effective Energy & Sub-Matrix & Regularization \\
\hline \hline 50 & 0.127 & 0.175 & 0.223 \\
70 & 0.150 & 0.111 & 0.133 \\
92 & 0.140 & 0.131 & 0.145 \\
\hline
\end{tabular}

Qualitatively, both the sub-matrix and regularization methods appear to better match the main peak present in the water form factor, although both have larger statistical uncertainties due to the matrix inversions. Both methods also sometimes give negative values for $F_{\text {coh }}^{2}$. This problem is exacerbated by the relatively large uncertainties in our measurements. 


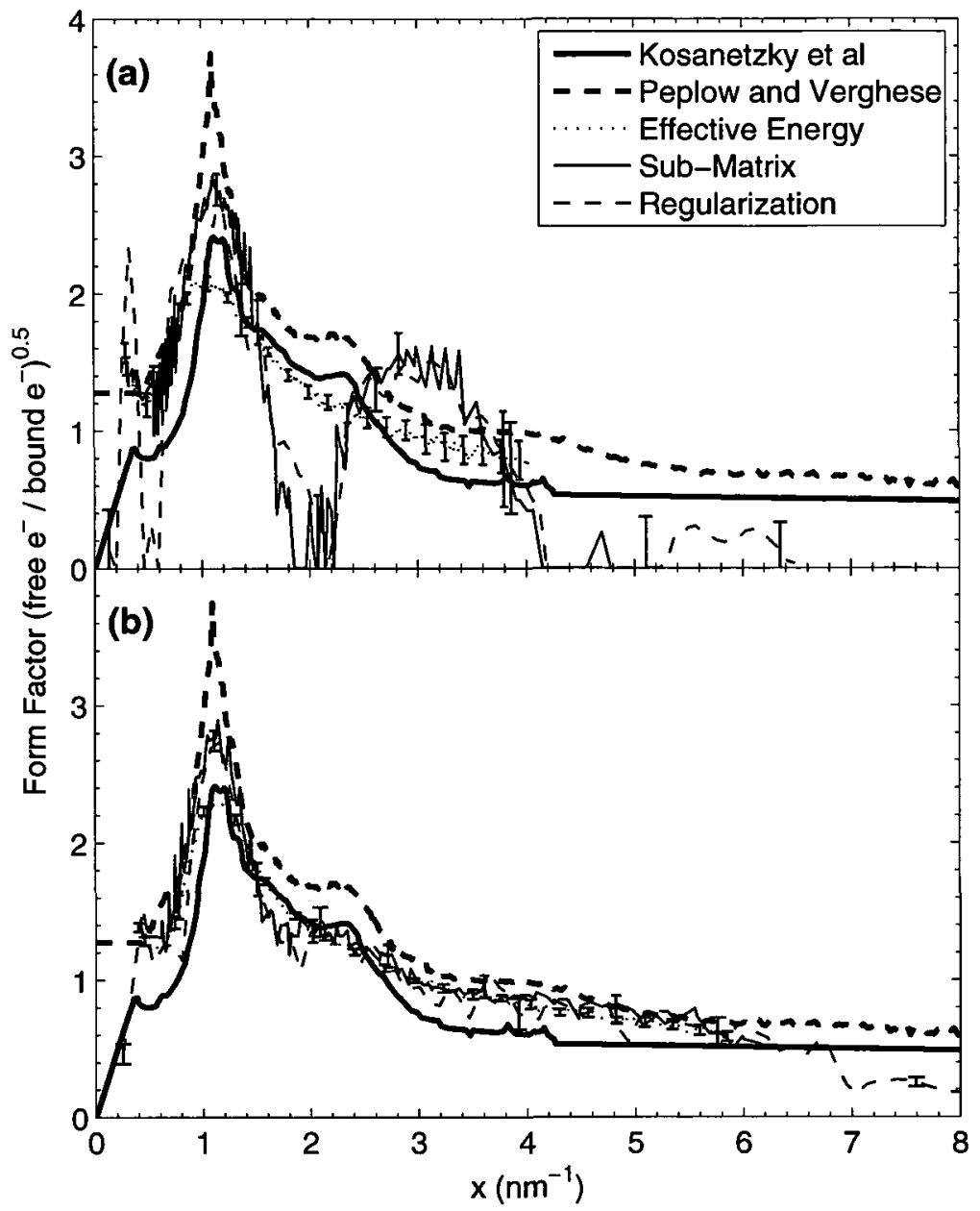

Figure 4.8: Form factor for fat extracted from measurements at (a) $50 \mathrm{kV}$ and (b) $70 \mathrm{kV}$. Error bars are shown for only every twentieth point for clarity. The Peplow and Verghese data are from beef fat measurements.

Form factors were calculated from the fat measurements shown in Figure 4.4. The results are shown in Figure 4.8 compared to previous measurements [20,21]. A quantitative comparison is made in Table 4.5. These measurements show peak form factors between the two extremes of Kosanetzky et al and Peplow and Verghese. The $50 \mathrm{kVp}$ results also show the same discrepancy at $2 \sim 3 \mathrm{~nm}^{-1}$ as the water measurements. Overall, considering the problems representing sharp peaks inherent in the sub-matrix and regularization calculations, our results seem to agree better with the data of Peplow and Verghese. The reason for the discrepancies between these measurements is not clear. 
Table 4.5: Average absolute relative difference $D_{\mathrm{av}}$ over the range 0.5 to 4.0 $\mathrm{nm}^{-1}$ of each calculated form factor for fat shown in Figure 4.8 as compared to reference data[20, 21]. All measured form factors were smoothed by 5 point rectangular averaging.

\begin{tabular}{lllll}
\hline $\begin{array}{l}\text { Potential } \\
(\mathrm{kV})\end{array}$ & Comparison & $\begin{array}{l}\text { Effective } \\
\text { Energy }\end{array}$ & Sub-Matrix & Regularization \\
\hline \hline 50 & Kosanetzky et al & 0.228 & 0.528 & 0.485 \\
50 & Peplow and Verghese & 0.199 & 0.207 & 0.242 \\
70 & Kosanetzky et al & 0.217 & 0.277 & 0.220 \\
70 & Peplow and Verghese & 0.163 & 0.126 & 0.179 \\
\hline
\end{tabular}

\subsection{Discussion}

The form factor measurement techniques developed in Chapter 3 and verified in this chapter are significantly different from previous angle-dispersive methods. We use a relatively large fraction of the full spectral range of the $\mathrm{x}$-ray tube, compared to the very restricted spectral range used in typical diffractometer experiments. Additionally, we use a large area detector that allows us to collect a much larger fraction of the scattered $\mathrm{x}$ rays. There are several obvious advantages that this type of detector provides. Since the signal in scatter experiments is usually quite weak, collecting as many photons as possible is very important. By collecting the average signal in each annular ring, we also improve the statistical uncertainty in our data. Unfortunately, due to the interexposure variation inherent in our $\mathrm{x}$-ray system, this improvement by signal averaging was insignificant compared to the overall uncertainty in the scatter signal.

In general, the results of the sub-matrix and regularization methods shown in Section 4.3 are quite similar. The regularization calculation seems to break down at the largest $x$ values. This appears to be inherent in the method itself as the same trend appears in the simulation results in Figure 3.8. 
The tube potentials and filters chosen for study in this chapter were selected to examine the progression from relatively light filtration (broad spectra) to heavy filtration (narrow spectra). The $92 \mathrm{kV}$ results for water, the heaviest filtration used in this experiment, show relatively small differences between the effective energy calculation and the two newer methods. This is consistent with the fact that the spectrum was much narrower, with strong tungsten fluorescence peaks present.

The $50 \mathrm{kV}$ spectrum is well suited to the particular detector used in this experiment. As seen in Figure 4.6, the quantum efficiency of the BaFBr drops off significantly above $40 \mathrm{keV}$. The $50 \mathrm{kV}$ spectrum would also be the most clinically accessible, requiring only aluminum filtration. In practice, however, we found that the most convenient tube voltage was $70 \mathrm{kV}$. This gave the clearest signal with the least exposure time, allowing for relatively quick measurements.

Uncertainties in the form factors were calculated assuming small uncertainties in the $\underline{\mathbf{P}_{\mathbf{x}}}$ elements compared to those from $S(\theta)$. This means that the form factor uncertainties are somewhat underestimated. However, we have verified that the results are very similar when slightly different spectra are used to perform the calculation.

The experimental setup used in this experiment was not ideal. Much of this was due to equipment limitations. The $\mathrm{x}$-ray tube output variation from one exposure to the next was an important factor, requiring significantly longer experiment times to repeat the exposures and degrading the accuracy level. This was part of the reason that fat measurements were not performed at $92 \mathrm{kV}$. The time required to perform multiple exposures would have led to the possibility of structural changes in the sample as the fat degraded.

The increased efficiency of x-ray collection in this geometry also makes the experiment more susceptible to multiply scattered $x$ rays. To the extent that any extraneous signal caused by multiple scatter or other sources was the same in both the signal and the background measurements, it can be subtracted with the background measurements. For low attenuation targets such as tissues, this approximation is mostly valid. However, in 
targets with larger attenuation factors, it is not.

An attempt was made to measure the form factor of a bone sample but this was unsuccessful due to the more significant effect of target attenuation. The bone scatter pattern was significantly attenuated because of the high target density. The same attenuation was not present in the background scatter pattern. This led to negative values for the measured scatter pattern at small angles. This effect would also have been present, but to a much smaller extent, in the water and fat exposures.

To reduce the effect of target attenuation, we propose a modified experimental configuration where the background and target vials are both in the beam path for each exposure. For the target exposure, the background vial would be placed directly in front of the $\mathrm{x}$-ray tube with the target vial in its normal position. For the background exposure, the vials would be interchanged. This would result in the same attenuation of the beam for both target and background. It could also contribute extra scatter. We suspect that this effect would be limited by the collimation in the system, but our experience has shown that quantitative scattering experiments can be quite sensitive.

An alternative method for determining an accurate background would be to calculate the form factor of the background vial from the background scatter measurements, then calculate what the background scatter pattern should be based on the attenuated spectrum. This could be feasible with a cleaner, stronger signal and potentially a higher resolution measurement using a sharper $\mathrm{x}$-ray spectrum. 


\section{CHAPTER 5}

\section{ENERGY-DISPERSIVE FORM FACTOR}

\section{MEASUREMENTS - DEVELOPMENT}

In this chapter, I investigate an alternative method of measuring coherent scattering form factors. Instead of an angle-dispersive configuration, I develop a model for an energy-dispersive configuration: measuring the scattering at a fixed scattering angle as a function of energy. From Equation 1.1, this still allows measurement of the form factor at a specific $x$ value.

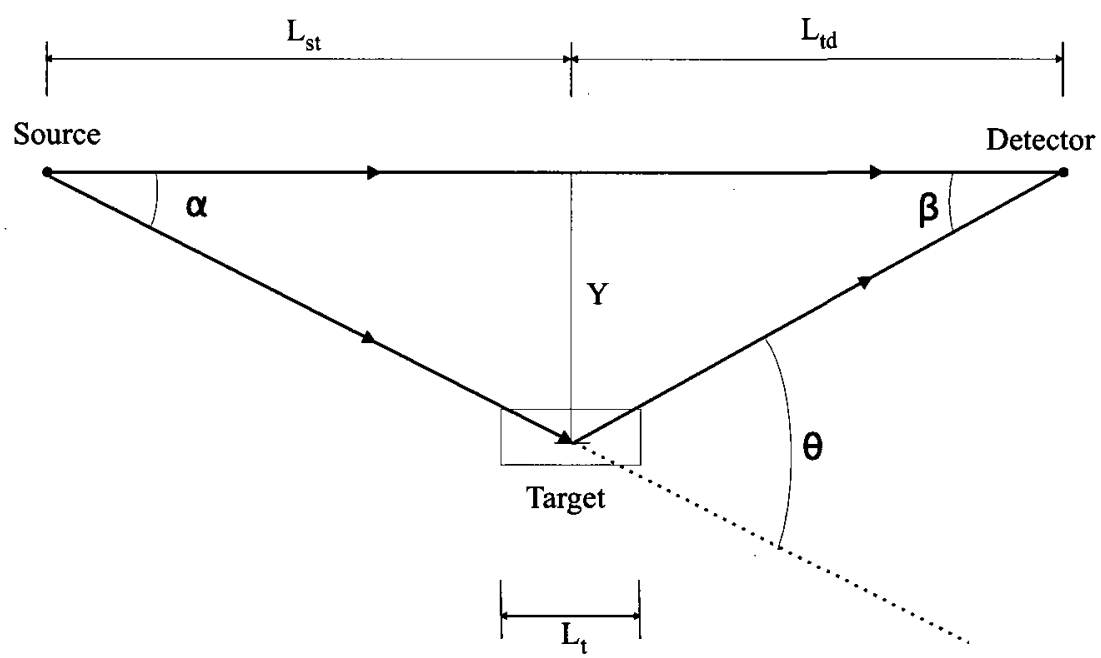

Figure 5.1: Schematic of experimental setup for energy-dispersive measurement.

In an energy-dispersive measurement, a strongly polychromatic source of $\mathrm{x}$ rays is an advantage, making this method much better suited to the output of an x-ray tube. An energy sensitive detector is required for which we used our HPGe spectrometer. We will 
show later in this chapter that measurement of the coherent scattering form factor requires spectra to be measured both at scattering angle $\theta$ and in a transmission configuration with no scattering. The geometry of Figure 5.1 allows both the transmission and scatter spectra to be measured by translating the target laterally without adjusting the source or detector. This configuration is based on earlier work by our group [24, 29].

\subsection{Apparatus}

A detailed plan of our experimental setup is shown in Figure 5.2. The target was placed in a custom-made target station with adjustable apertures both before and after the target to allow a number of different $\theta$ to be measured. By measuring at different $\theta$, the range of $x$ accessible with our method was extended.

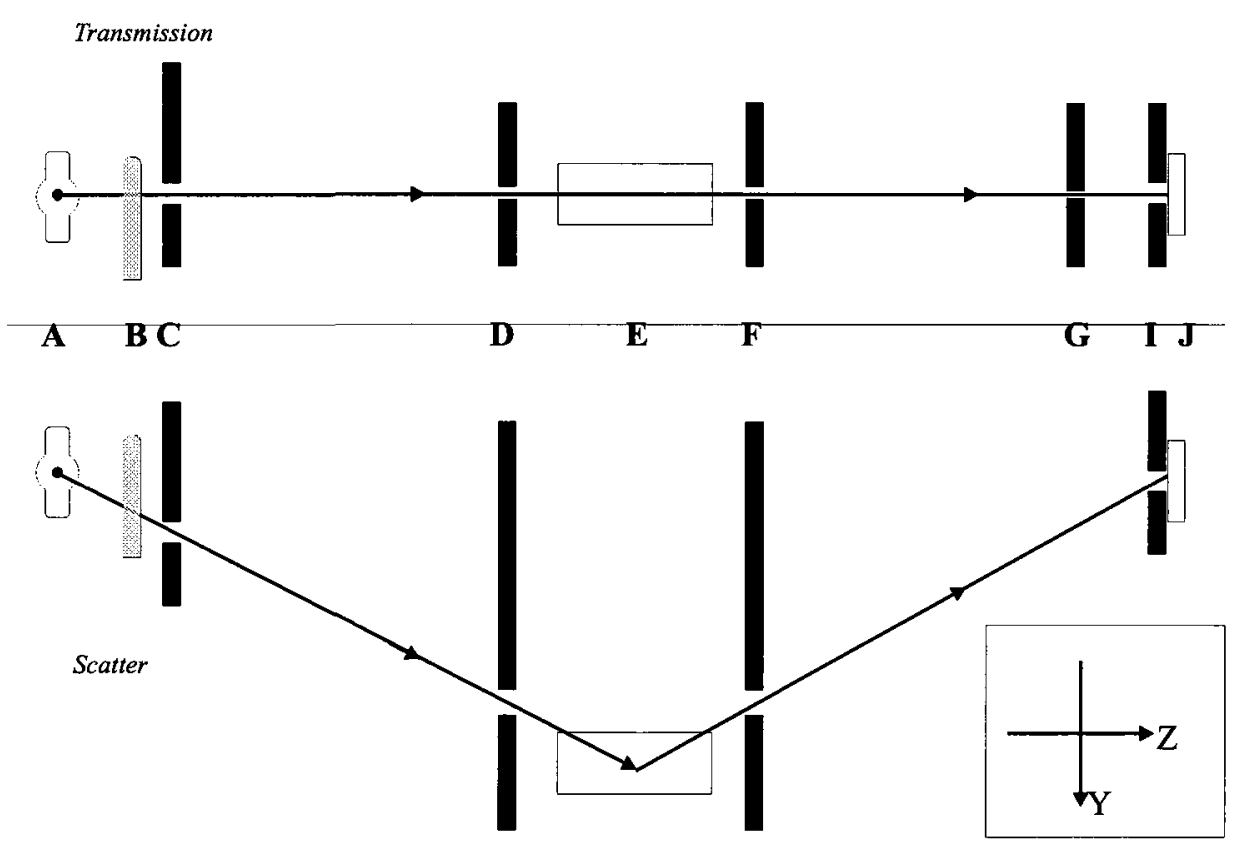

Figure 5.2: Detailed experimental setup showing apertures for form factor measurements in transmission and scatter configurations. All equipment except the X-ray tube (A), ion chamber (B) and spectrometer (J) were mounted on an optical bench for precise positioning and alignment. Apertures $C$ and $F$ can be translated laterally to align with different apertures at plane $D$. The location and dimension of each item can be found in Table 5.1. 
Table 5.1: Location of objects in transmission and scatter configuration as shown in Figure 5.2. The $\mathrm{Z}$ position refers to the centre of each object. For more information on the transmission aperture $\mathrm{G}$ see section 5.1.1.

\begin{tabular}{llcl}
\hline Object & Description & Z Position $(\mathrm{mm})$ & Thickness $(\mathrm{mm})$ \\
\hline \hline A & Source & 0 & N/A \\
B & Ion chamber & $128 \pm 6$ & N/A \\
C & Aperture & $138 \pm 6$ & $2.0 \pm 0.1$ \\
D & Aperture & $645 \pm 3$ & $3.9 \pm 0.1$ \\
E & Target & $693 \pm 4$ & $40.0 \pm 0.5$ \\
F & Aperture & $769 \pm 3$ & $3.9 \pm 0.1$ \\
G & Aperture & $1293 \pm 6$ & See text \\
I & Aperture & $1451 \pm 3$ & $3.9 \pm 0.1$ \\
J & Detector & $1458 \pm 6$ & N/A \\
\hline
\end{tabular}

Table 5.2: Available aperture dimensions used for the energy-dispersive form factor measurements.

\begin{tabular}{lllc}
\hline Punch & Shape & W Width $(\mathrm{mm})$ & H Height $(\mathrm{mm})$ \\
\hline \hline $\mathrm{C} 1$ & Circular & $1.66 \pm 0.03$ & $1.66 \pm 0.03$ \\
$\mathrm{C} 2$ & Circular & $3.32 \pm 0.05$ & $3.32 \pm 0.05$ \\
$\mathrm{R} 1$ & Rectangular & $2.08 \pm 0.04$ & $7.66 \pm 0.03$ \\
$\mathrm{R} 2$ & Rectangular & $3.12 \pm 0.07$ & $7.62 \pm 0.06$ \\
$\mathrm{R} 3$ & Rectangular & $3.09 \pm 0.04$ & $10.11 \pm 0.09$ \\
\hline
\end{tabular}

\subsubsection{Apertures}

All apertures in the system, except aperture $\mathrm{G}$ in Figure 5.2, were punched out of $\mathrm{Pb}$ sheet with a Di-Acro mechanical punch (Acrotech, Lake City, MN). The dimensions were measured with a travelling microscope and are given in Table 5.2. 
Aperture plane $\mathrm{D}$ was punched with a circular aperture for the transmission configuration plus seven different rectangular apertures, allowing seven different scatter configurations. Apertures $\mathrm{C}$ and $\mathrm{F}$ can be translated laterally to align with any one of these holes. This allows for independent measurements of the form factor in different $x$ regions. The punches used in each configuration are shown in Table 5.3. Narrower apertures were used for the first four scatter configurations to improve the resolution at small $x$ where more structure is expected for amorphous materials. Wider apertures were used for the last three scatter configurations to improve the counting statistics.

Table 5.3: Aperture configurations used for form factor measurements. The detector aperture in the transmission configuration is defined by a separate transmission aperture $(\mathrm{G})$. The dimensions of the apertures can be found in Table 5.2.

\begin{tabular}{lllll}
\hline Configuration & Aperture C & Aperture D & Aperture F & Aperture I \\
\hline \hline Transmission & C2 & C1 & R1 & N/A \\
Scatter 1 & C2 & R1 & R1 & R1 \\
Scatter 2 & C2 & R1 & R1 & R1 \\
Scatter 3 & C2 & R1 & R1 & R1 \\
Scatter 4 & C2 & R1 & R1 & R1 \\
Scatter 5 & C2 & R2 & R2 & R3 \\
Scatter 6 & C2 & R2 & R2 & R3 \\
Scatter 7 & C2 & R2 & R2 & R3 \\
\hline
\end{tabular}

Optimal alignment of aperture $C$ was accomplished by using a PRM model D-15 pancake style ion chamber (Precision Radiation Measurement Inc., Nashville, TN) in place of the target at plane $\mathrm{E}$ and measuring the exposure for each configuration as the aperture was translated laterally.

Optimal alignment of aperture $\mathrm{F}$ was accomplished using a thin $(6.6 \mathrm{~mm}$ thick) water target and measuring the total number of counts observed by the HPGe detector for each 
configuration as the aperture was translated laterally. It is important that a thin target be used to ensure that the optimal value is found for the centre of the target. If a thicker target is used, multiple scattering can cause a false optimum to be found for larger scattering angles.

For measurements in the transmission configuration, where the fluence from the primary beam was very high, an additional aperture (labelled G in Figure 5.2) was used to limit the count rate. This aperture comprises a $5 \mathrm{~mm}$ thick $\mathrm{Pb}$ sheet with a small pinhole (made with a nominal $0.5 \mathrm{~mm}$ drill) followed by an opposed pair of $6 \mathrm{~mm}$ diameter tungsten carbide tipped micrometer spindles mounted directly behind the pinhole. The effective area of the pinhole referenced to aperture plane I was determined by computing the ratio of the total counts measured by the spectrometer through the pinhole to those measured through a larger aperture with known dimensions, incorporating the inverse square law. A $65 \mathrm{kVp}$ spectrum with $3 \mathrm{~cm} \mathrm{Al} \mathrm{filtration} \mathrm{at} \mathrm{low} \mathrm{current} \mathrm{was} \mathrm{used} \mathrm{to} \mathrm{keep} \mathrm{the}$ countrate low.

The tungsten acts as a very effective beam blocker and the micrometer spindles allow precise control of the aperture size for a very small aperture. The effective area of the aperture, referenced to plane I and denoted by $A_{\mathrm{dt}}$, was measured by computing a further ratio of counts in each energy bin at $121 \mathrm{kVp}$, measured at low current, with the spindles in the open position and in their final position. Ideally, the ratio would be energy independent, giving a constant value for $A_{\mathrm{dt}}$. In practice, the tungsten spindles add a slight energy dependence which is compensated for by fitting to the measured area as a function of energy. This ensures that the tungsten spindles do not affect the measured form factors. The effective area of the aperture corresponds roughly to a rectangular aperture of width $0.02 \mathrm{~mm}$ and height $0.5 \mathrm{~mm}$. The effective area can vary slightly each time the aperture is emplaced so $A_{\mathrm{dt}}$ was characterized before each transmission spectrum was measured using the function bkFitAttenSpectra2 (see Appendix C). A typical result of the measurement of $A_{\mathrm{dt}}$ is shown in Figure 5.3. For measurements in the scatter configuration, the transmission 
aperture was removed from the bench completely.

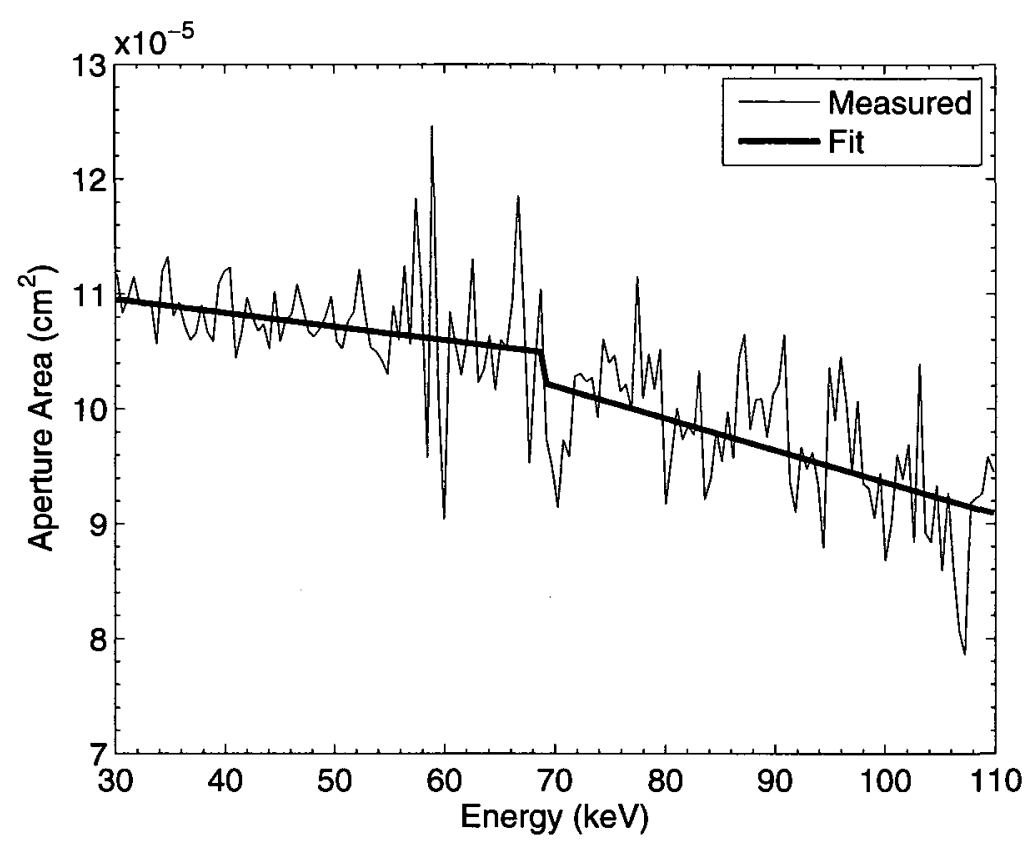

Figure 5.3: Typical energy dependence of effective transmission aperture area caused by tungsten spindles. The fitting function is computed from separate straight lines in the energy ranges $30-69 \mathrm{keV}$ and $69-110 \mathrm{keV}$. Note that the tungsten $\mathrm{K}$-edge is at $69 \mathrm{keV}$.

\subsubsection{X-ray tube}

The $\mathrm{x}$-ray tube was a rotating $\mathrm{W}$ anode tube with a Machlett-Dynamax $69 \mathrm{M}$ insert, powered by a Picker GX550 single phase generator. It was operated at $121 \mathrm{kV}$ and $2 \mathrm{~mA}$ using the nominal $0.6 \mathrm{~mm}$ focal spot for a total exposure of approximately $600 \mathrm{mAs}$. The tube was mounted vertically such that the heel effect did not affect the tube spectrum as seen at different scattering angles. The tube has a target angle of $12^{\circ}$ but was tilted toward the detector by $9.83^{\circ}$ to reduce the apparent height of the focal spot through foreshortening. The output of the $\mathrm{x}$-ray tube is monitored using a PTW N30001, $0.6 \mathrm{~cm}^{3}$ cylindrical ion chamber (PTW Freiburg, Germany) placed directly in front of the x-ray tube, out of the beam path for the measurements. The ion chamber was kept stationary throughout the ex- 
periments. The ion chamber measurements were used to normalize all measured spectra to the same tube output.

\subsubsection{HPGe Detector}

All spectra were measured using an Ortec planar HPGe detector (Ortec, Oak Ridge, TN), with a detector crystal $16 \mathrm{~mm}$ in diameter and $10 \mathrm{~mm}$ thick. The crystal is located behind a $0.5 \mathrm{~mm} \mathrm{Be}$ window. The detector was located in a $\mathrm{Pb}$ casing to reduce the effect of ambient radiation. $\mathrm{A} \mathrm{Pb}$ aperture, labelled $\mathrm{I}$ in Figure 5.2, was placed in the casing to limit the area of the detector exposed to radiation and to ensure that the radiation was absorbed in the central region of the crystal. The dimensions of the apertures are given in Table 5.3. The signal chain consisted of a Canberra 2024 spectroscopy amplifier, 8077 ADC, and an Aptec 2000 series MCA, all from Canberra (Canberra Industries, Meriden CT). The MCA was integrated into a personal computer.

\subsubsection{Target Station}

The target station is shown in Figure 5.4. The target was placed on the plastic target holder which can be translated laterally using the precision slide assembly (Velmex, Bloomfield, NY). Liquid targets were placed in a $40 \mathrm{~mm} \times 22 \mathrm{~mm} \times 28 \mathrm{~mm}$ rectangular container made from polycarbonate with $0.5 \mathrm{~mm}$ thick entrance and exit windows. The container fits securely in place on the target holder to ensure a consistent target location. Solid targets (of dimension $40 \mathrm{~mm} \times 10 \mathrm{~mm} \times 20 \mathrm{~mm}$ ) were placed directly on the target holder.

\subsubsection{Spectral Measurements}

Some of the x-ray photons that reach the detector will not come from scattering within the target. In order to remove the effect of these photons, background spectra were measured for each scattering angle. There are two distinct sources of unwanted photons that must be treated separately: First, the number of ambient photons that were present throughout 


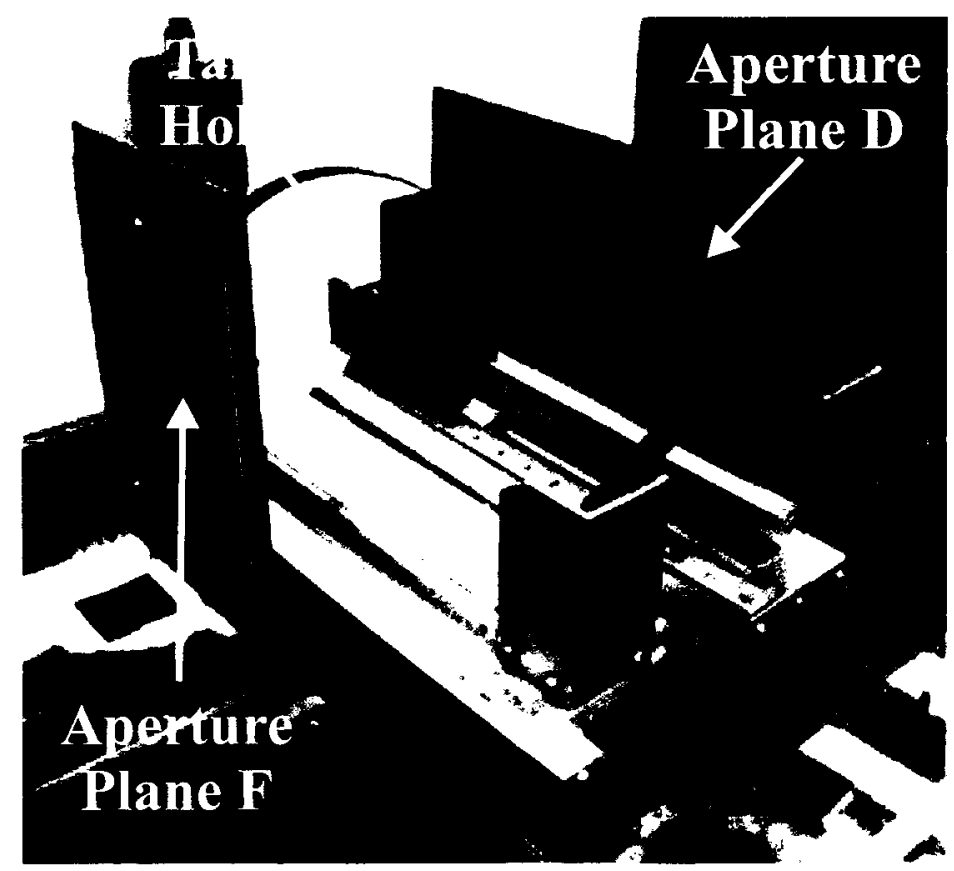

Figure 5.4: Photograph of the target station. During measurements, a single hole in each of aperture planes $\mathrm{D}$ and $\mathrm{F}$ was uncovered. All other apertures were covered by $4 \mathrm{~mm}$ of $\mathrm{Pb}$ and additional $\mathrm{Pb}$ sheets were placed around the sides and top to prevent any stray radiation from escaping.

the room was determined by blocking all of the holes in aperture $\mathrm{D}$ and measuring the spectrum. Second, the number of background photons that scattered along the path of the beam, in the walls of the target container, or from the edges of the apertures, was determined by measuring the spectrum with an empty target container. In this case, the spectrum measured was corrected for the attenuation that these background photons would experience when travelling through the target in the actual experiment. Assuming small scattering angles such that the distance travelled through the target was simply $L_{t}$, the transmission factor was determined by measuring the spectra along the transmission path with the target present and without.

Taken altogether, this means that five spectra were required in order to compute the coherent scattering form factor with a given configuration: two spectra along the transmission path, one with the target present and one without, two spectra along the scatter path, one with the target present and one without, and one spectrum to measure the ambient 
room scatter. All measured spectra were normalized to the tube monitoring ion chamber signal.

Three spectral effects could cause artifacts in the measured form factors. The spectra were not corrected for the detector response of the HPGe spectrometer. This effect is insignificant for Ge spectrometers except at the low energy cutoff where additional photons are measured. The transmission measurements, with their much higher count rates showed a small amount of pulse pileup at the high energy limit. Additionally, the region around the tungsten characteristic peaks is quite sensitive to the energy calibration of the detector and the precise energy assigned to these peaks. This can lead to large measurement errors if the energies of the peaks are not perfectly stable. To avoid complications from these artifacts, only the energy range $30-110 \mathrm{keV}$ was used in the form factor calculation. As well, energies between $57-60 \mathrm{keV}$ and $67-70 \mathrm{keV}$ were excluded to avoid the tungsten characteristic peaks. We verified using an earlier prototype version of our experiment that pileup was insignificant over this energy range by measuring the form factor using different tube currents. No appreciable differences were found.

\subsection{Theory}

Here, we derive expressions for the number of photons measured by the detector in each energy range $E \rightarrow E+d E$ in both the transmission and scatter configurations.

In the transmission configuration, there is no appreciable scattering and the detector will measure the direct, transmission spectrum. If the differential incident fluence per energy interval at distance $L_{\mathrm{st}}$ is $\mathrm{d} \Phi_{\mathrm{t} 0} / \mathrm{d} E$, then in the energy range $E \rightarrow E+\mathrm{d} E$, the detector will measure

$$
\mathrm{d} N_{\mathrm{t}}(E)=\mathrm{d} \Phi_{\mathrm{t} 0}(E) \frac{L_{\mathrm{st}}^{2}}{\left(L_{\mathrm{st}}+L_{\mathrm{td}}\right)^{2}} A_{\mathrm{dt}} \exp \left[-\mu_{\mathrm{t}}(E) L_{\mathrm{t}}\right]
$$

where $\mu_{\mathrm{t}}$ is the attenuation coefficient of the target and $A_{\mathrm{dt}}$ is the effective area of the 
detector exposed to the source in transmission mode as determined, including energy dependence, in Section 5.1.1.

For the scattering configurations, when $Y>0$ in Figure 5.1 and the differential incident fluence per energy interval at distance $L_{\mathrm{st}}$ is $\mathrm{d} \Phi_{\mathrm{s} 0} / \mathrm{d} E$, the differential incident x-ray fluence reaching a particular depth $l$ of the target is

$$
\frac{\mathrm{d} \Phi_{\mathrm{s}}}{\mathrm{d} E}=\frac{\mathrm{d} \Phi_{\mathrm{s} 0}}{\mathrm{~d} E} \frac{L_{\mathrm{st}}^{2}}{\left(L_{\mathrm{st}}^{2}+Y^{2}\right)} \exp \left[-\frac{\mu_{\mathrm{t}}(E) l}{\cos \alpha}\right]
$$

The probability of these photons scattering at depth $l$ into a given solid angle $\Delta \Omega$ and travelling through the rest of the target is

$$
p_{\mathrm{s}}(l)=\frac{\mathrm{d}_{\mathrm{e}} \sigma}{\mathrm{d} \Omega} \frac{\rho_{\mathrm{e}} \mathrm{d} V(l)}{A_{\mathrm{t}}(l) \cos \alpha} \exp \left[-\frac{\mu_{\mathrm{t}}(E)\left(L_{\mathrm{t}}-l\right)}{\cos \beta}\right] \Delta \Omega
$$

Here $A_{\mathrm{t}}(l)$ is the area of the target at depth $l$ from which scattering can be observed at the detector, $\rho_{\mathrm{e}}$ is the electron density of the target material, $\mathrm{d} V(l)=A_{\mathrm{t}}(l) \mathrm{d} l$ is an element of target volume at depth $l$, and the solid angle of the detector is given by

$$
\Delta \Omega=\frac{A_{\mathrm{ds}} \cos \beta}{L_{\mathrm{td}}^{2}+Y^{2}}
$$

where $A_{\mathrm{ds}}$ is the physical area of the detector aperture I corrected for its finite thickness for photons incident at angle $\beta$. The differential number of photons, $\mathrm{d}^{2} N_{\mathrm{s}}$, observed by the detector in the energy range $E \rightarrow E+\mathrm{d} E$ from the layer $\mathrm{d} l$ at depth $l$ is $d \Phi_{\mathrm{s}} A_{\mathrm{t}}(l) \cos \alpha p_{\mathrm{s}}(l)$ :

$$
\begin{aligned}
\mathrm{d}^{2} N_{\mathrm{s}} & =\frac{\mathrm{d} \Phi_{\mathrm{s} 0}(E) L_{\mathrm{st}}^{2} \rho_{\mathrm{e}} A_{\mathrm{ds}} \cos \beta}{\left(L_{\mathrm{st}}^{2}+Y^{2}\right)\left(L_{\mathrm{td}}^{2}+Y^{2}\right)} \exp \left[-\frac{\mu_{\mathrm{t}}(E) L_{\mathrm{t}}}{\cos \beta}\right] \frac{\mathrm{d}_{\mathrm{e}} \sigma}{\mathrm{d} \Omega} \\
& \times \exp \left[-\mu_{\mathrm{t}}(E) l\left(\frac{1}{\cos \alpha}-\frac{1}{\cos \beta}\right)\right] A_{\mathrm{t}}(l) \mathrm{d} l
\end{aligned}
$$

The total number of photons observed by the detector is given by integrating this expres- 
sion over all depths:

$$
\begin{aligned}
\mathrm{d} N_{\mathrm{s}}(E) & =\frac{\mathrm{d} \Phi_{\mathrm{s} 0}(E) L_{\mathrm{st}}^{2} \rho_{\mathrm{e}} A_{\mathrm{ds}} \cos \beta}{\left(L_{\mathrm{st}}^{2}+Y^{2}\right)\left(L_{\mathrm{td}}^{2}+Y^{2}\right)} \exp \left[-\frac{\mu_{\mathrm{t}}(E) L_{\mathrm{t}}}{\cos \beta}\right] \frac{\mathrm{d}_{\mathrm{e}} \sigma}{\mathrm{d} \Omega} \\
& \times \int_{0}^{L_{\mathrm{t}}} \exp \left[-\mu_{\mathrm{t}}(E) l\left(\frac{1}{\cos \alpha}-\frac{1}{\cos \beta}\right)\right] A_{\mathrm{t}}(l) \mathrm{d} l
\end{aligned}
$$

The exponential factor in the integral is very nearly 1 for the small (roughly equal) angles $\alpha$ and $\beta$ present in our case and can be neglected. The integral then resolves to simply the scattering volume within the target, $V_{\mathrm{t}}=\int A_{\mathrm{t}}(l) \mathrm{d} l$.

The ratio of $\mathrm{d} N_{\mathrm{s}}(E)$ to $\mathrm{d} N_{\mathrm{t}}(E)$ in each energy range $E \rightarrow E+\mathrm{d} E$ is then

$$
\frac{\mathrm{d} N_{\mathrm{s}}(E)}{\mathrm{d} N_{\mathrm{t}}(E)}=\left[\frac{\left(L_{\mathrm{st}}+L_{\mathrm{td}}\right)^{2} \rho_{\mathrm{e}} V_{\mathrm{t}} \cos \beta}{\left(L_{\mathrm{st}}^{2}+Y^{2}\right)\left(L_{\mathrm{td}}^{2}+Y^{2}\right)}\right]\left[\frac{A_{\mathrm{ds}}}{A_{\mathrm{dt}}}\right]\left[\frac{\mathrm{d} \Phi_{\mathrm{s} 0}}{\mathrm{~d} \Phi_{\mathrm{t} 0}}\right] \exp \left[-\mu_{\mathrm{t}}(E) L_{\mathrm{t}}\left(\frac{1}{\cos \beta}-1\right)\right] \frac{\mathrm{d}_{\mathrm{e}} \sigma}{\mathrm{d} \Omega}
$$

For small $\beta$, the exponential factor can be neglected and so using the definition of the scattering cross-section from equation 2.19 , an expression for the coherent scattering form factor is arrived at based entirely on measurable and tabulated parameters:

$$
\begin{aligned}
F_{\mathrm{coh}}(x) & =\left\{\left[\frac{\left(L_{\mathrm{st}}^{2}+Y^{2}\right)\left(L_{\mathrm{td}}^{2}+Y^{2}\right)}{\left(L_{\mathrm{st}}+L_{\mathrm{td}}\right)^{2} \frac{\mathrm{d}_{\mathrm{e}} \sigma_{0}}{\mathrm{~d} \Omega} \rho_{\mathrm{e}} V_{\mathrm{t}} \cos \beta}\right]\left[\frac{A_{\mathrm{dt}}}{A_{\mathrm{ds}}}\right]\left[\frac{\mathrm{d} \Phi_{\mathrm{t} 0}}{\mathrm{~d} \Phi_{\mathrm{s} 0}}\right]\left[\frac{\mathrm{d} N_{\mathrm{s}}(E)}{\mathrm{d} N_{\mathrm{t}}(E)}\right]\right. \\
& \left.-F_{\mathrm{KN}}(E, \theta) F_{\mathrm{inc}}(x)\right\}^{1 / 2}
\end{aligned}
$$

For small scattering angles, the incoherent contribution is negligible. For water at a scattering angle of $15^{\circ}$ however, based on Figure 2.6, the incoherent contribution varies from $30 \%$ at $30 \mathrm{keV}$ to $85 \%$ at $110 \mathrm{keV}$.

\subsubsection{Parameter calculation}

The scattering volume $V_{\mathrm{t}}$ as well as the scattering angles cannot be measured directly in the experiment. Instead, I have developed a Monte Carlo ray-tracing simulation of the experiment in Matlab (The Mathworks, Natick, MA). A Monte Carlo integration of the 
volume was computed by uniformly sampling $N$ points within a rectangular parallelepiped target volume $V_{\text {tot }}$. For each, a point is chosen within the acceptance area of the detector $A_{\mathrm{ds}}$ (this acceptance area depends on $\beta$ because of transmission effects through the thick aperture directly in front of the detector), and a point is chosen from the spatial distribution of the x-ray tube fluence $\Phi_{\mathrm{s}}$ over the focal spot area $A_{\mathrm{s}}$. We modelled $\Phi_{\mathrm{s}}$ with a twodimensional normal distribution with widths determined from pinhole images of the focal spot[42]. The number of points $N_{\mathrm{t}}$ for which the path defined by the source, target and detector points passes through all of the apertures in the system was then calculated. The target volume $V_{\mathrm{t}}$ was then obtained as $V_{\mathrm{t}}=N_{\mathrm{t}} / N \cdot V_{\text {tot }}$ in the limit of large $N$. Strictly speaking, because we have included the finite sizes of the source and detector in the Monte Carlo calculation, the integral actually calculated is

$$
\int_{V_{\mathrm{t}}} \int_{A_{\mathrm{s}}} \int_{A_{\mathrm{ds}}} \mathrm{d} A \mathrm{~d} \Phi \mathrm{d} V=\frac{N_{\mathrm{t}}}{N} V_{\mathrm{tot}} \Phi_{\mathrm{s}} A_{\mathrm{ds}}
$$

so that the volume calculated is actually an effective volume for a system with fluence $\Phi_{\mathrm{s}}$ and detector area $A_{\mathrm{ds}}$. Since it is the total product that appears in the expression for $F_{\mathrm{coh}}$, this difference does not affect the calculated result. For more details on Monte Carlo integration, see chapter 4 of Reference [43]. This volume calculation is similar to that of [44] with the exception that we have extended the calculation to model the effect of rectangular apertures with finite thickness. The effect of thick apertures can be quite significant for the larger angles. As an example, for configuration number $7, V_{\mathrm{t}}$ is increased by almost $50 \%$ if all of the apertures are assumed to be thin.

The calculation treats all apertures as ideal, ignoring any penetration through the collimator edges. The volume calculation has a precision better than $0.5 \%$. The same Monte Carlo calculation is also used to determine $\alpha, \beta, \theta$, and the offset $Y$ as well as the statistical uncertainties and correlations of all parameters. There are 9 geometric parameters appearing directly in Equation 5.8 and 29 geometric parameters overall. Uncertainties in 
Table 5.4: Calculated parameters for scattering apertures from Monte Carlo calculation. The calculated uncertainties include variations in all experimental parameters.

\begin{tabular}{llllll}
\hline Configuration & $\alpha(\mathrm{deg})$ & $\beta(\mathrm{deg})$ & $\theta(\mathrm{deg})$ & $Y(\mathrm{~cm})$ & $V_{\mathrm{t}}\left(\mathrm{cm}^{3}\right)$ \\
\hline \hline Scatter 1 & $0.91 \pm 0.11$ & $0.77 \pm 0.09$ & $1.67 \pm 0.17$ & $1.07 \pm 0.11$ & $0.61 \pm 0.13$ \\
Scatter 2 & $1.70 \pm 0.11$ & $1.48 \pm 0.09$ & $3.17 \pm 0.15$ & $2.04 \pm 0.11$ & $0.51 \pm 0.10$ \\
Scatter 3 & $2.67 \pm 0.12$ & $2.36 \pm 0.08$ & $5.03 \pm 0.15$ & $3.22 \pm 0.12$ & $0.39 \pm 0.05$ \\
Scatter 4 & $3.33 \pm 0.12$ & $2.97 \pm 0.09$ & $6.30 \pm 0.15$ & $4.03 \pm 0.11$ & $0.31 \pm 0.03$ \\
Scatter 5 & $5.31 \pm 0.13$ & $4.74 \pm 0.11$ & $10.05 \pm 0.17$ & $6.45 \pm 0.11$ & $0.42 \pm 0.03$ \\
Scatter 6 & $6.63 \pm 0.13$ & $5.95 \pm 0.11$ & $12.58 \pm 0.17$ & $8.07 \pm 0.12$ & $0.31 \pm 0.02$ \\
Scatter 7 & $7.94 \pm 0.13$ & $7.15 \pm 0.11$ & $15.09 \pm 0.17$ & $9.69 \pm 0.11$ & $0.244 \pm 0.017$ \\
\hline
\end{tabular}

all of the parameters are factored into the uncertainty estimates. The calculated values are given in Table 5.4.

\subsubsection{Overall Fluence ratio}

The Monte Carlo calculation of Section 5.2.1 models the finite size of the $\mathrm{x}$-ray tube focal spot but it does not include any variations in the size or strength of the $x$-ray beam for different $\alpha$. We accounted for this by measuring the overall fluence ratio $d \Phi_{\mathrm{t} 0} / \mathrm{d} \Phi_{\mathrm{s} 0}$ separately.

We measured the exposure from the x-ray tube with a PRM model D-15 pancake style ion chamber (Precision Radiation Measurement Inc., Nashville, TN) in place of the target at plane $\mathrm{E}$ for each configuration in Table 5.3. The signal in each case is proportional to the fluence at the ion chamber and to the effective volume, $v$ of the ion chamber visible through the apertures. In the transmission configuration,

$$
S_{\mathrm{t}} \propto \frac{\mathrm{d} \Phi_{\mathrm{t} 0}}{L_{\mathrm{st}}^{2}} \mathrm{v}_{\mathrm{t}}
$$


while in the scatter configuration,

$$
S_{\mathrm{s}} \propto \frac{\mathrm{d} \Phi_{\mathrm{s} 0}}{L_{\mathrm{st}}^{2}+Y^{2}} \mathbf{v}_{\mathrm{s}}
$$

Taking the ratio of Equations 5.10 and 5.11 allows an expression to be derived for the ratio $\mathrm{d} \Phi_{\mathrm{t} 0} / \mathrm{d} \Phi_{\mathrm{s} 0}$ :

$$
\frac{\mathrm{d} \Phi_{\mathrm{t} 0}}{\mathrm{~d} \Phi_{\mathrm{s} 0}}=\frac{\left(\frac{L_{\mathrm{st}}^{2}}{L_{\mathrm{st}}^{2} Y^{2}}\right) \frac{\mathrm{v}_{\mathrm{s}}}{\mathrm{v}_{\mathrm{t}}}}{\frac{S_{\mathrm{s}}}{S_{\mathrm{t}}}} \equiv \frac{r_{\mathrm{e}}}{r_{\mathrm{m}}} .
$$

The ratio $r_{\mathrm{e}}$ is the expected scatter to transmission ratio under the assumption that the $\mathrm{x}$-ray tube fluence is independent of $\alpha$. The ratio $r_{\mathrm{m}}=S_{\mathrm{s}} / S_{\mathrm{t}}$ is the actual scatter to transmission ratio measured with the ion chamber. The expected ratio was calculated using a similar Monte Carlo calculation to that in Section 5.2.1 using the function bkfluencefactor2 (see Appendix C.1). The focal spot was modelled using the same dimensions as in Section 5.2.1 for consistency.

The overall fluence ratios measured using this procedure are shown in Table 5.5. The ratio decreases with larger angles, meaning that the $\mathrm{x}$-ray tube fluence increases for the larger scattering angles. This somewhat surprising result is not fully understood, although the effect is quite repeatable. The assumption with this procedure is that any variation in $\mathrm{x}$-ray tube output is not energy dependent as the ion chamber is energy insensitive. If this assumption were not true, then form factors measured with the different configurations would be inconsistent. 
Table 5.5: Measured fluence ratios $\left(\mathrm{d} \Phi_{\mathrm{t} 0} / \mathrm{d} \Phi_{\mathrm{s} 0}\right)$ for scattering apertures. Measurements for the ratios were performed three times and averaged. The increase in $r_{\mathrm{e}}$ and $r_{\mathrm{m}}$ between Scatter 4 and Scatter 5 is due to the difference in the aperture sizes, as given in Table 5.3.

\begin{tabular}{llll}
\hline Aperture & $r_{\mathrm{e}}$ & $r_{\mathrm{m}}$ & $\mathrm{d} \Phi_{\mathrm{t} 0} / \mathrm{d} \Phi_{\mathrm{s} 0}$ \\
\hline \hline Scatter 1 & $7.2 \pm 0.3$ & $7.50 \pm 0.12$ & $0.96 \pm 0.04$ \\
Scatter 2 & $7.0 \pm 0.3$ & $7.83 \pm 0.07$ & $0.90 \pm 0.04$ \\
Scatter 3 & $6.7 \pm 0.3$ & $7.26 \pm 0.06$ & $0.93 \pm 0.04$ \\
Scatter 4 & $6.6 \pm 0.3$ & $7.78 \pm 0.10$ & $0.84 \pm 0.04$ \\
Scatter 5 & $9.7 \pm 0.4$ & $11.55 \pm 0.07$ & $0.84 \pm 0.04$ \\
Scatter 6 & $9.3 \pm 0.4$ & $11.03 \pm 0.11$ & $0.85 \pm 0.04$ \\
Scatter 7 & $8.9 \pm 0.4$ & $10.9 \pm 0.2$ & $0.82 \pm 0.04$ \\
\hline
\end{tabular}




\section{CHAPTER 6}

\section{ENERGY-DISPERSIVE FORM FACTOR}

\section{MEASUREMENTS - RESULTS}

In this chapter, I implement the energy dispersive calculations of Chapter 5 and show results of the energy-dispersive form factor measurements for water, fat and several types of plastics. Most of the measurements in this section were made by Karl Landheer, under my supervision.

\subsection{Results}

The energy-dispersive form factors were calculated from Equation 5.8 using the function bkendispFFCalc4 (see Appendix C.1). The spectra measured with a water target for each configuration are shown in Figure 6.1. We can see that, with the exception of the first scatter configuration, there is no appreciable $\mathrm{Pb} \mathrm{K}$-edge in any of the spectra. This suggests that $\mathrm{Pb}$ penetration through the edges of the apertures is not a significant effect and that the assumption of ideal apertures in the Monte Carlo simulations of Section 5.2.1 is reasonable. The $\mathrm{Pb} \mathrm{K}$-edge in the first configuration is present in the background as well, suggesting that this is an effect of primary contamination due to the very small angles present in this configuration.

While performing the measurements for this thesis, a slight shift in the measured form factors $\left(<0.05 \mathrm{~nm}^{-1}\right)$ was observed as compared to our preliminary results. This was 


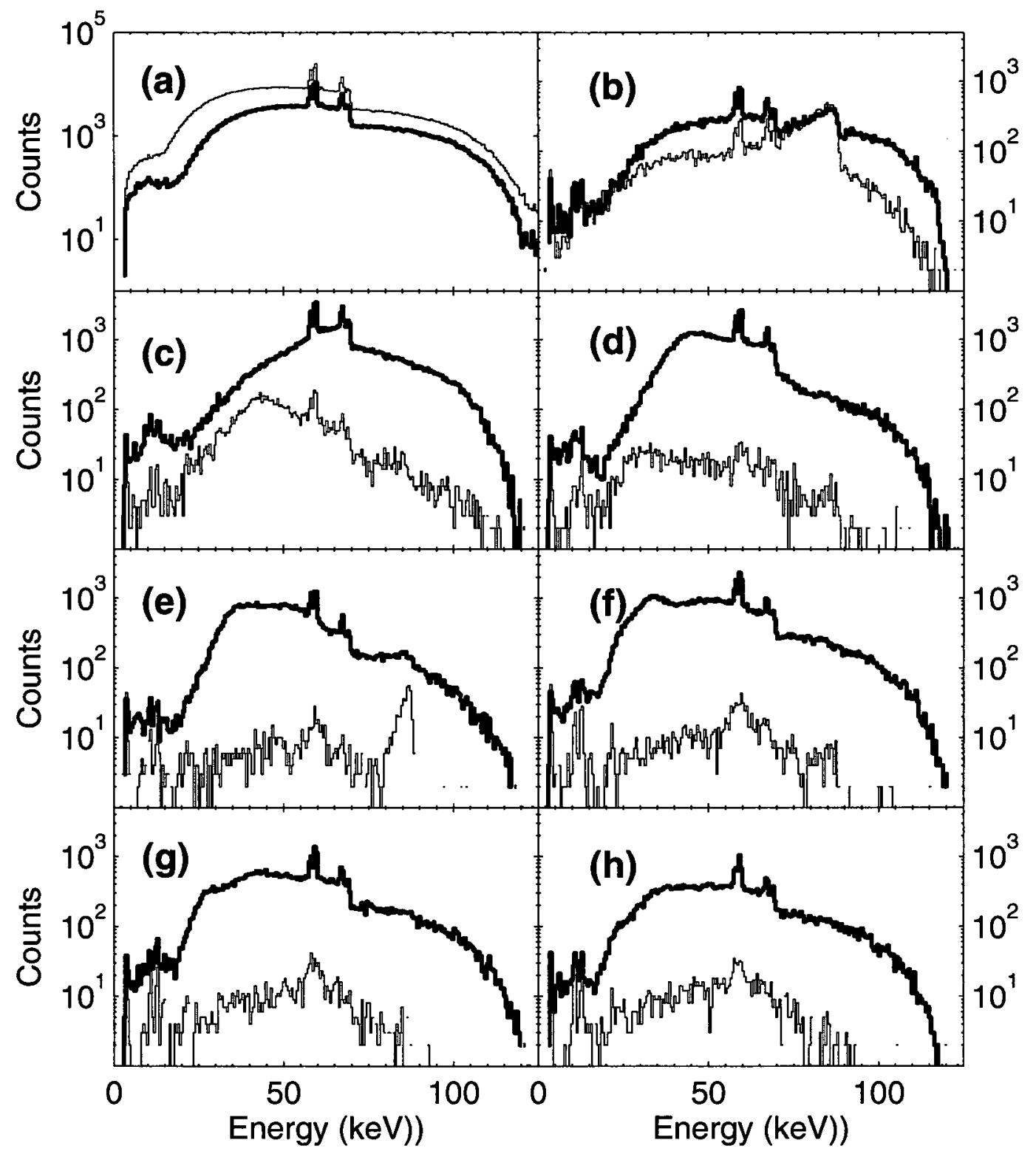

Figure 6.1: Spectra measured for each configuration in Table 5.3 with a water target. Spectra were measured with the target present (dark line) and without (light line). Panel (a) shows the transmission spectra while (b) through (h) show scatter spectra 1 through 7 , in order. The spectra shown here are not normalized to the ion chamber measurements. 
determined to be a result of a small lateral shift in the location of the detector. The size of the movement was measured by observing the displacement of the detector aperture (I) compared to a laser beam aligned along the transmission path. An offset of $1.0 \mathrm{~mm}$ was observed for configurations $1-4$ and $1.5 \mathrm{~mm}$ for configurations 5-7. These offsets were included in the Monte Carlo model of Section 5.2.1 to ensure that the angle and volume calculations were modelling the true experimental configuration.

In order to determine $F_{\text {coh }}$ from Equation 5.8, we must know $\rho_{\mathrm{e}}$ and $F_{\text {inc }}$ for the material. These can be computed from the chemical composition and density, $\rho$. The electron density $\rho_{\mathrm{e}}$ can be determined from the mean atomic number, $\bar{Z}$ and atomic weight, $\bar{A}$ :

$$
\rho_{\mathrm{e}}=\rho N_{A} \overline{\bar{A}},
$$

where $N_{A}$ is Avogadro's number, $6.022 \times 10^{23}$. Values for $F_{\text {inc }}$ were obtained from Reference [17]. The required information for water, fat and the plastics studied in this chapter are given in Table 6.1.

Table 6.1: Compositions and parameters of water, fat, and plastics. The density of water at $22.5^{\circ} \mathrm{C}$ was taken from page 6-7 of Reference [45] while the plastic densities were measured directly. The composition and density of fat were taken from Reference [46].The uncertainty range for the water measurement covers the range of temperatures expected over the experiment while the uncertainty range for fat is based on the differences in fat density in the literature.

\begin{tabular}{llll}
\hline Material & Chemical Formula & Density $\left(\mathrm{g} \mathrm{cm}^{-3}\right)$ & $\rho_{\mathrm{e}}\left(\mathrm{cm}^{-3}\right)$ \\
\hline \hline water & $\mathrm{H}_{2} \mathrm{O}$ & $0.9976 \pm 0.0005$ & $(3.3348 \pm 0.0017) \times 10^{23}$ \\
fat & See Reference $[46]$ & $0.92 \pm 0.02$ & $(3.09 \pm 0.06) \times 10^{23}$ \\
polyethylene & $\left(\mathrm{C}_{2} \mathrm{H}_{4}\right)_{\mathrm{n}}$ & $0.948 \pm 0.005$ & $(3.255 \pm 0.016) \times 10^{23}$ \\
nylon & $\left(\mathrm{C}_{6} \mathrm{H}_{11} \mathrm{NO}\right)_{\mathrm{n}}$ & $1.138 \pm 0.004$ & $(3.753 \pm 0.015) \times 10^{23}$ \\
polystyrene & $\left(\mathrm{C}_{8} \mathrm{H}_{8}\right)_{\mathrm{n}}$ & $1.042 \pm 0.004$ & $(3.373 \pm 0.013) \times 10^{23}$ \\
polycarbonate & $\left(\mathrm{C}_{16} \mathrm{H}_{14} \mathrm{O}_{3}\right)_{\mathrm{n}}$ & $1.17 \pm 0.01$ & $(3.71 \pm 0.04) \times 10^{23}$ \\
\hline
\end{tabular}




\subsubsection{Water Measurements}

Based on these parameters, the experimental geometry and the measured spectra, we measured the coherent scattering form factor of water to verify that the energy dispersive method was configured correctly. All spectra were originally measured with bin sizes approximately $0.1 \mathrm{keV}$ wide. To improve the counting statistics, these raw spectra were rebinned into larger bins. The size of the bins was chosen such that the spectra for all configurations were measured on a consistent $x$ mesh. The form factors were measured on a 200 point logarithmic spaced mesh ranging from $0.25 \mathrm{~nm}^{-1}$ to $14 \mathrm{~nm}^{-1}$. This allowed finer resolution at small $x$ where more structure is expected.

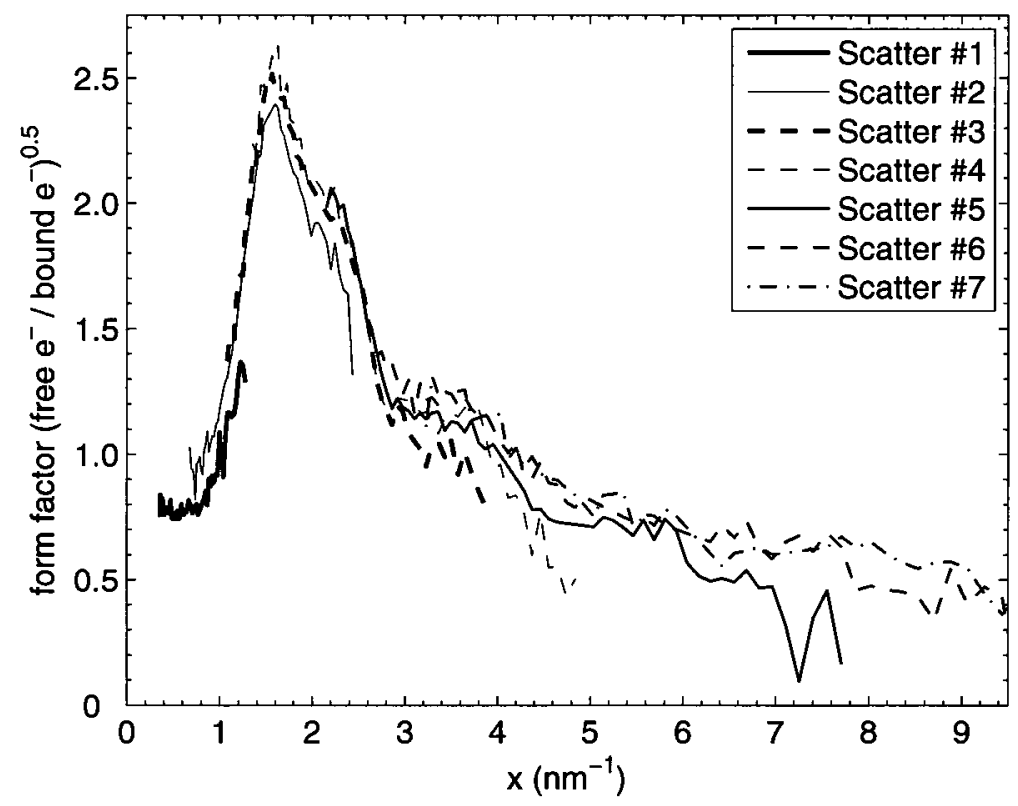

Figure 6.2: Form factor measurements for water from each scattering configuration. Error bars are not shown for clarity.

The individual form factor measurements from each scattering configuration for water are shown in Figure 6.2. We see that where different configurations measure the same $x$ values, good agreement is obtained. Since there are no adjustable parameters in our measurement, this suggests that our calculations have been implemented correctly. The 
measured value for each configuration drops off toward zero at large $x$ as the upper energy limit of the $\mathrm{x}$-ray spectrum is approached.

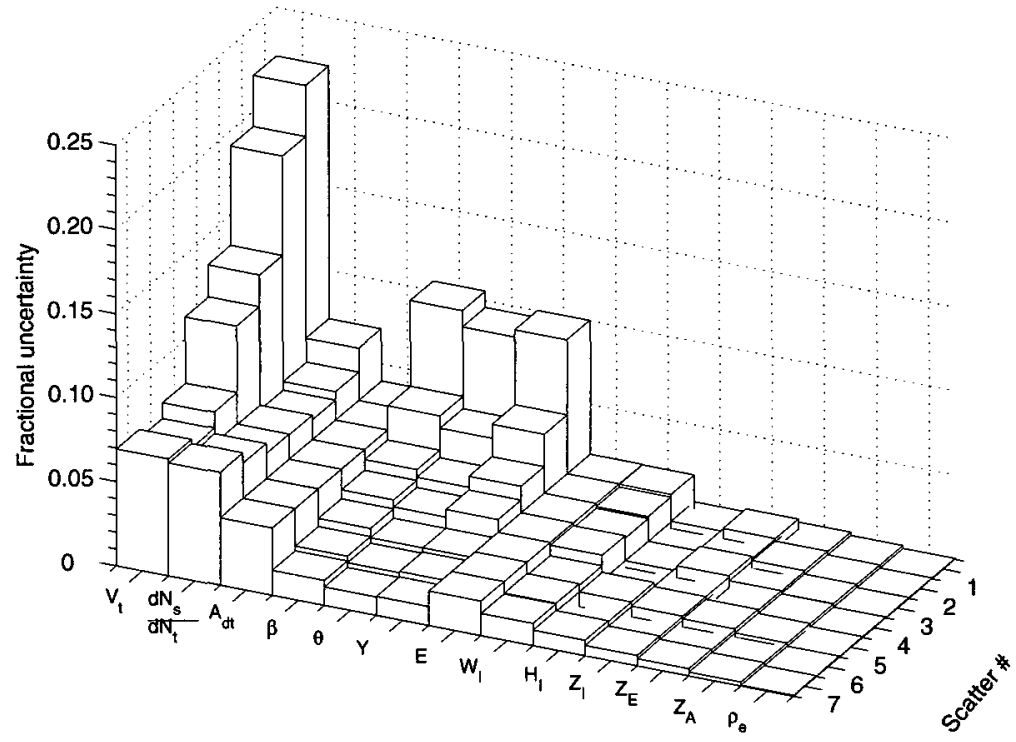

Figure 6.3: Uncertainties in experimental parameters for each configuration used in the measurement of the form factor of water at $50 \mathrm{keV}$. The subscripts on the $\mathrm{W}, \mathrm{H}$ and $\mathrm{Z}$ parameters refer to the aperture planes in Figure 5.2. The uncertainty in $\mathrm{d} N_{\mathrm{s}} / \mathrm{d} N_{\mathrm{t}}$ contains the effect of the fluence ratio as well. All parameters, with the exception of the ratio of the two measured spectra $\mathrm{d} N_{\mathrm{s}} / \mathrm{d} N_{\mathrm{t}}, A_{\mathrm{dt}}$ and $E$ itself are energy independent. Uncertainties in $F_{\text {inc }}$ were not included in the calculations.

Uncertainties were calculated for each point, taking into account uncertainties in all experimental parameters. A covariance matrix containing all of the geometric uncertainties was constructed by repeating the Monte Carlo calculation of Section 5.2.1 200 times as the experimental parameters were varied. Variances of the ratio $\mathrm{d} N_{\mathrm{s}} / \mathrm{d} N_{\mathrm{t}}$ and the effective transmission aperture area were determined using counting statistics. The covariance matrix was used to determine uncertainties for both $x$ and $F_{\text {coh. }}$. The uncertainties in each experimental parameter at a specific energy are shown in Figure 6.3. It can be seen from this that the most significant source of error in the form factor measurements comes from variation in $V_{\mathrm{t}}$. 
The uncertainty in $x$ is dominated for small scattering angles by the angular resolution and for large scattering angles by the energy resolution. The calculated errors in $x$ are shown in Figure 6.4 and for $F_{\text {coh }}$ in Figure 6.5.

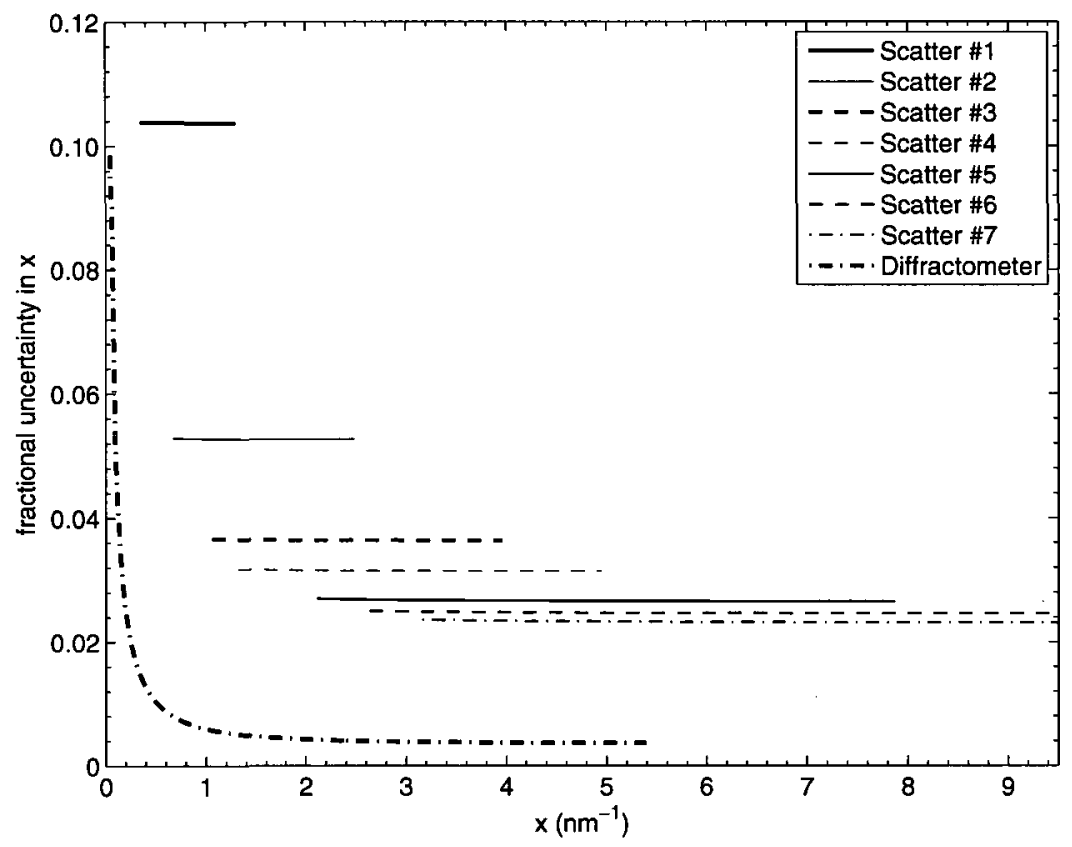

Figure 6.4: Resolution of $x$ for individual scattering apertures compared to Rigaku diffractometer results from Section 3.1.1 of Reference [23].

We used a long target $(4 \mathrm{~cm})$ to maximize the scatter countrate for the smaller scattering angles. The long length of the water sample means that the possibility of multiple scattering cannot be ignored. This effect would be most significant for the larger scattering angles where the scattering volume becomes smaller. We verified that multiple scattering was not significant by measuring the form factor for scatter configuration \#7 using a .66 $\mathrm{cm}$ and a $2.25 \mathrm{~cm}$ thick water target and comparing the results to the IAM value and to our large target measurements. The results are shown in Figure 6.6. We found that multiple scattering is not a significant issue, even with a $4 \mathrm{~cm}$ long target. 


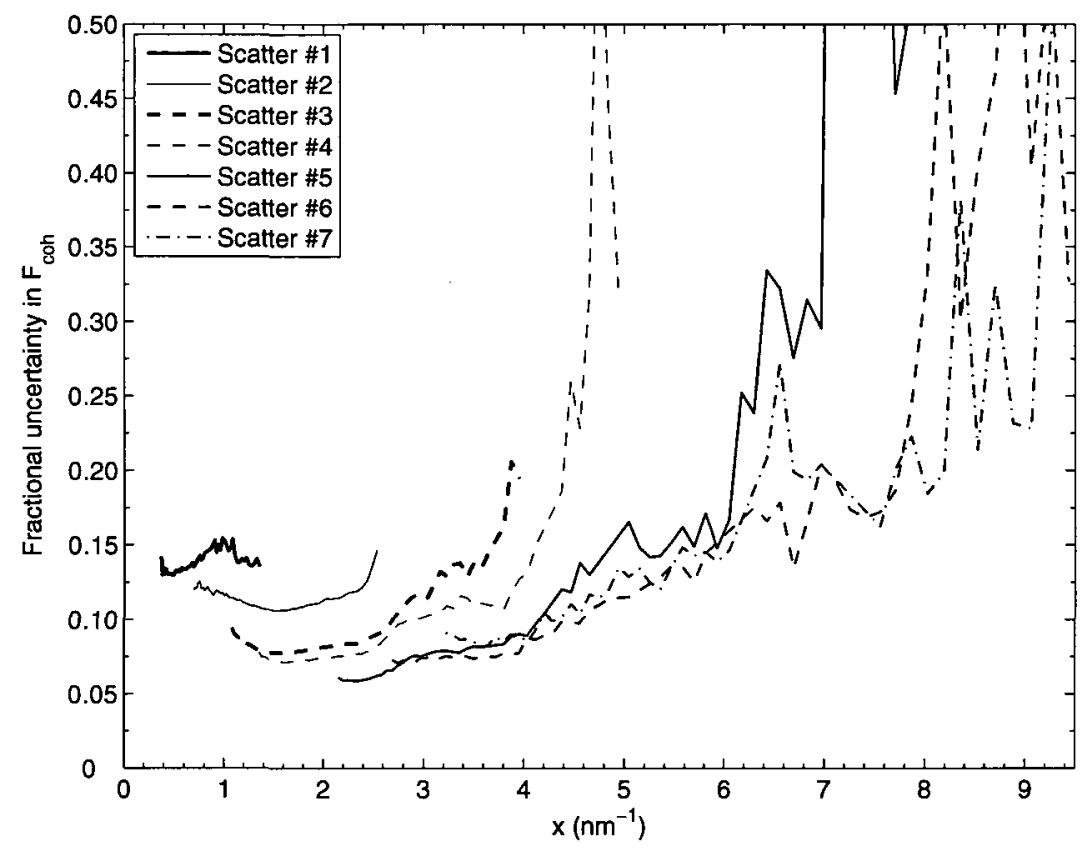

Figure 6.5: Uncertainty estimates for individual coherent scattering form factor measurements of water.

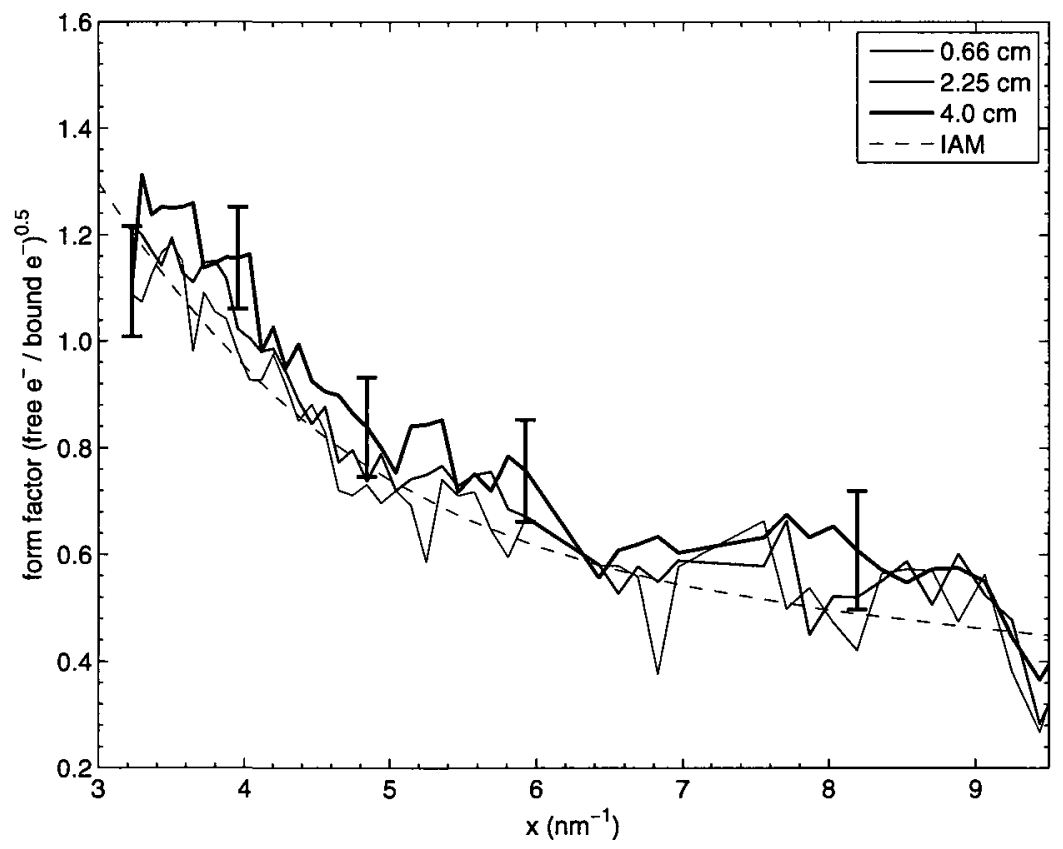

Figure 6.6: Measured form factors of water with three different target thicknesses. Error bars are only shown for a small number of points for the $4 \mathrm{~cm}$ thick target for clarity. Uncertainties for the $0.66 \mathrm{~cm}$ thick target are more than twice as large. 


\subsubsection{Plastic Measurements}

We also measured the form factor for a more structured material, polyethylene to examine the limits of the $x$ resolution of our equipment. Figure 6.7 shows the results for the range of $x$ from 0 to $5 \mathrm{~nm}^{-1}$. We see again that where the measurements overlap, good agreement was obtained, except around $1.2 \mathrm{~nm}^{-1}$ where the two sharp peaks visible with configuration 3 were blurred together for configurations 1 and 2. As seen in Figure 6.4, the $x$ resolution improves with larger scattering angles, explaining this difference.

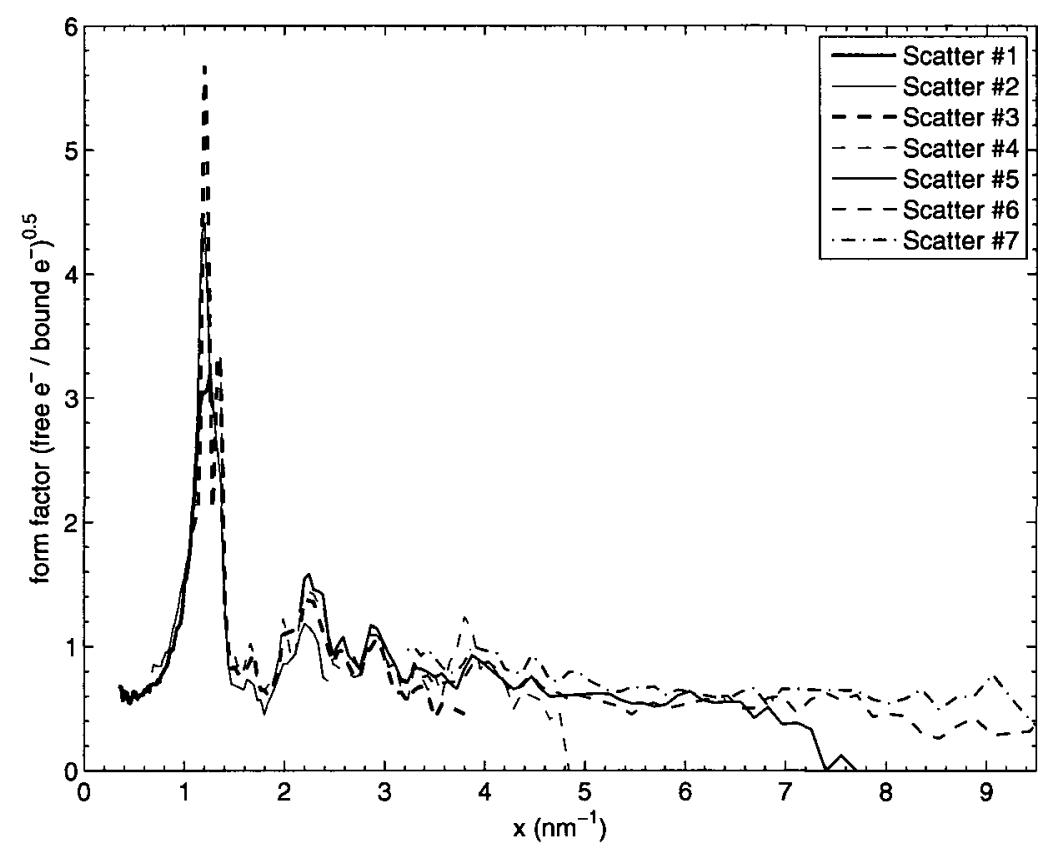

Figure 6.7: Form factor measurements for polyethylene from each scattering aperture between 0 and $5 \mathrm{~nm}^{-1}$. Error bars are omitted for clarity.

\subsubsection{Fat measurements}

Fat samples were prepared from beef samples from a butcher. Samples were refrigerated before use and were prepared immediately prior to performing the measurements. The samples were placed in the same containers that were used for the water measurement and covered with phosphate buffered saline (PBS) solution to prevent tissue dehydration. Any 
air in the beam path, with its very low electron density, would drastically affect the scatter signal and the measured form factor. To prevent this, we used a single piece of fat, cut as closely as possible to the container dimensions. The results are shown in Figure 6.8.

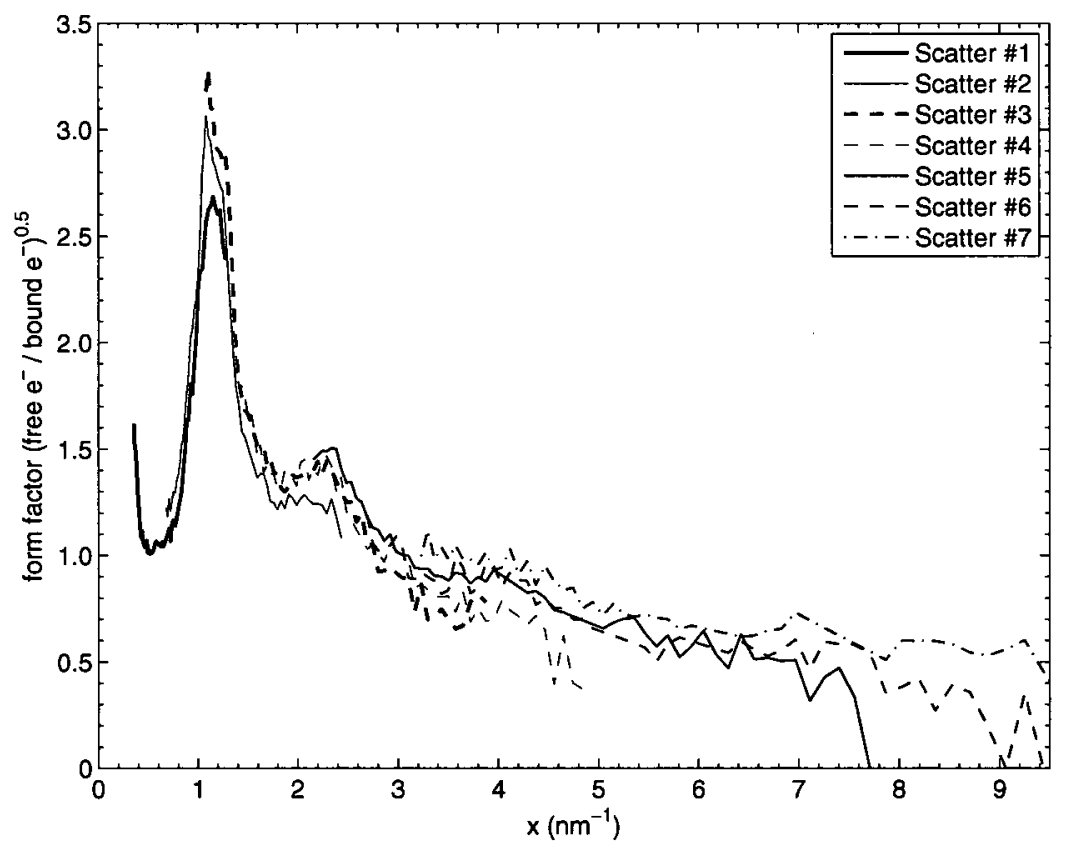

Figure 6.8: Form factor measurements for fat from each scattering aperture. Error bars are omitted for clarity.

\subsubsection{Combining individual measurements}

In order to determine the true form factor for each material, the individual measurements made with each configuration need to be combined together. The energy bins of our individual measurements were chosen such that the form factor measurements were on a consistent scale for all configurations. We therefore performed a $\chi^{2}$ test to check our individual measurements at each $x$ value for consistency with a $95 \%$ confidence interval. Consistent values were combined using a weighted average based on the uncertainty of each measurement.

For points where the $\chi^{2}$ test shows that the results are inconsistent, we did one of two things. For a single isolated $x$ value, we computed an unweighted average of the individual 
data points. If an interval in $x$ (defined as three or more adjacent points) was inconsistent at each $x$ value, then for each point we used the single measurement with the highest $x$ resolution under the assumption that the inconsistency was caused by a difference in resolution, such as with polyethylene.

The combined results for water are shown in Figure 6.9 compared to previously published values. Water has been studied by several groups, most extensively by Narten [19]. Although some differences exist between the measurements by different groups, the general behaviour of the scattering form factor is known. Polyethylene measurements are compared to those made using a diffractometer by Harding's group [20] in figure 6.10.

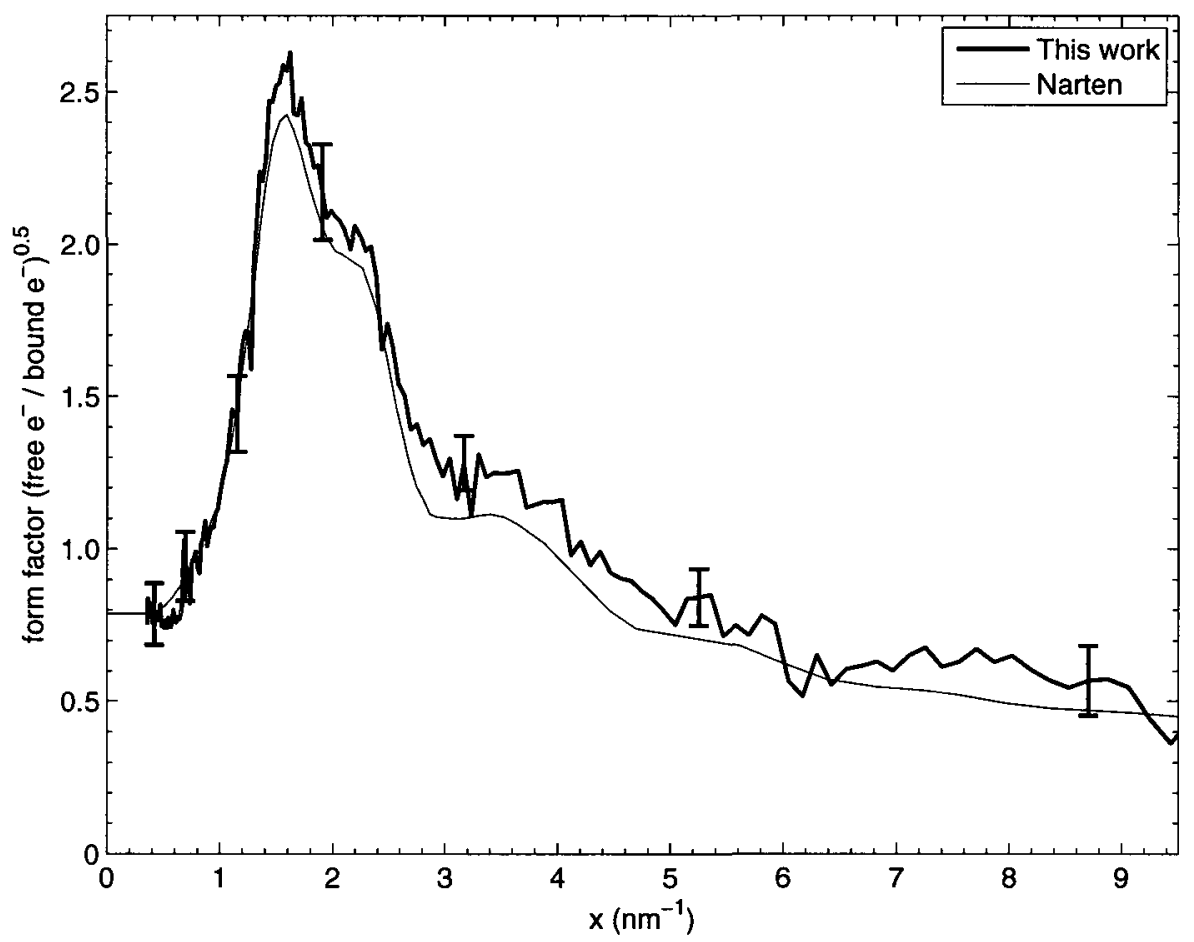

Figure 6.9: Combined form factor measurements of water compared to published values at $25^{\circ} \mathrm{C}$ (from Reference [19]). Error bars are only shown for every 25 th point for clarity.

The combined fat measurements in Figure 6.11 showed similar behaviour as the angledispersive measurements of Chapter 4, in the middle of the range of literature values. We show the results of further plastic measurements in Figure 6.12. Good agreement is found. 


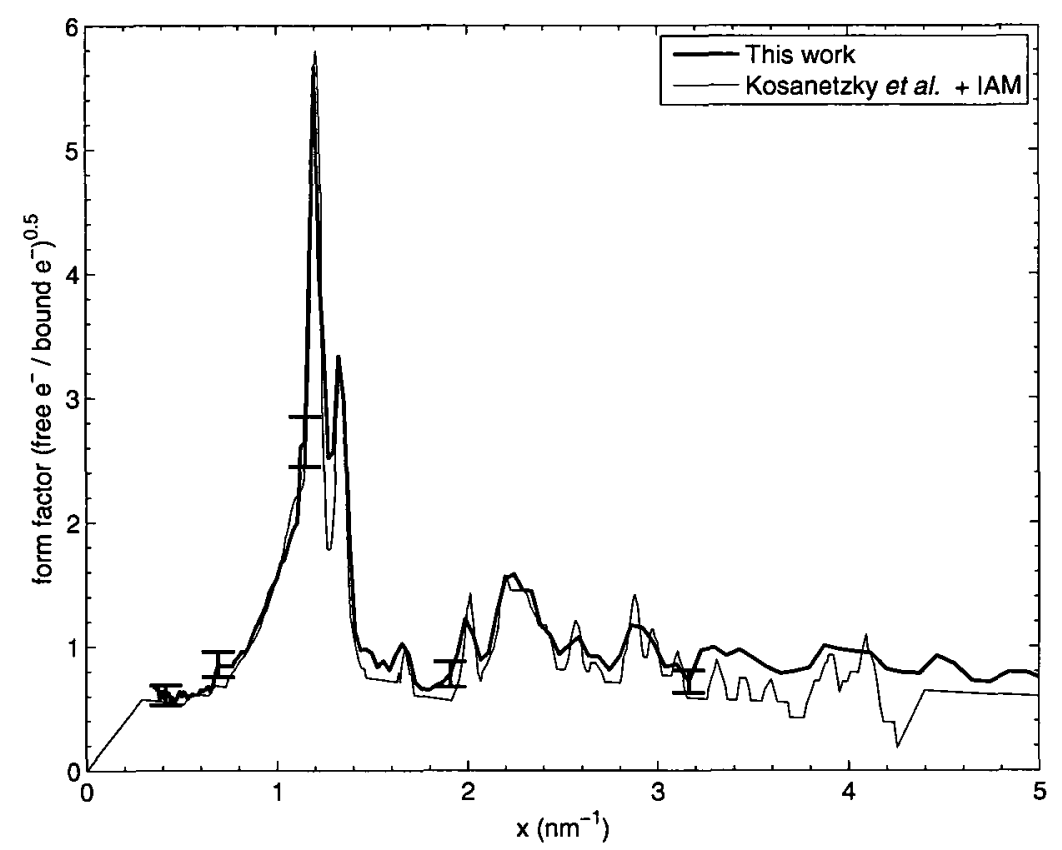

Figure 6.10: Combined form factor measurements of polyethylene compared to published values [20] over the range $0-5 \mathrm{~nm}^{-1}$. Error bars are only shown for every 25 th point for clarity.

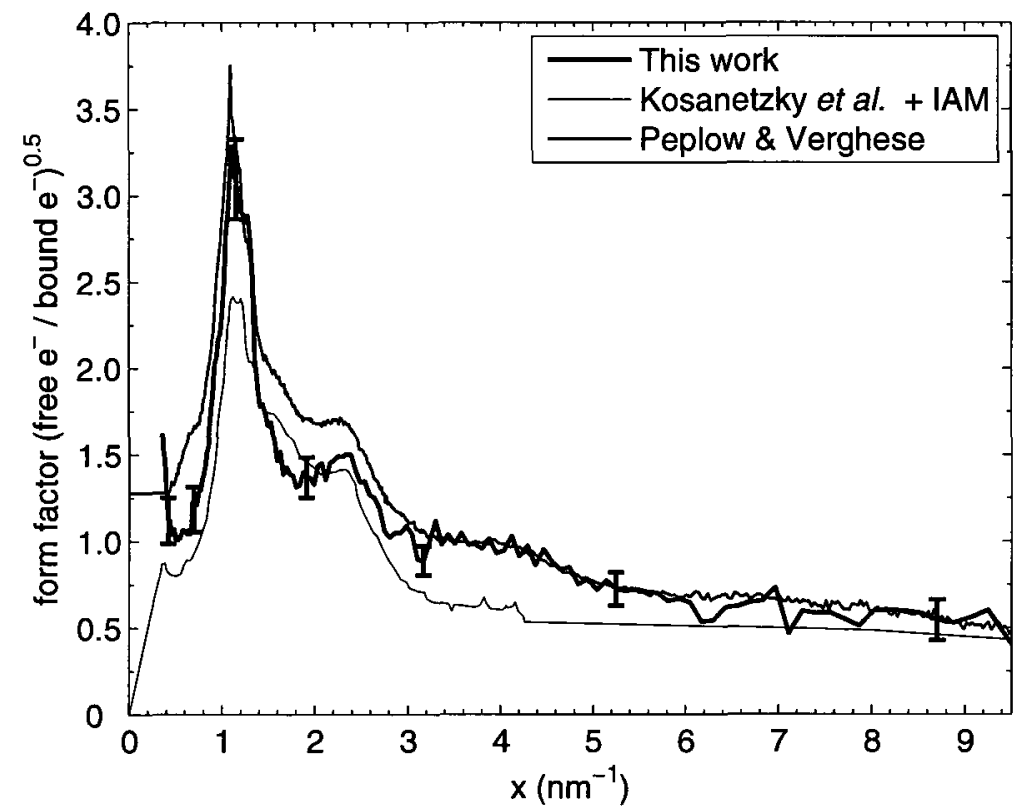

Figure 6.11: Combined form factor measurements of fat compared to published values [20, 21]. Error bars are only shown for every 25 th point for clarity. 


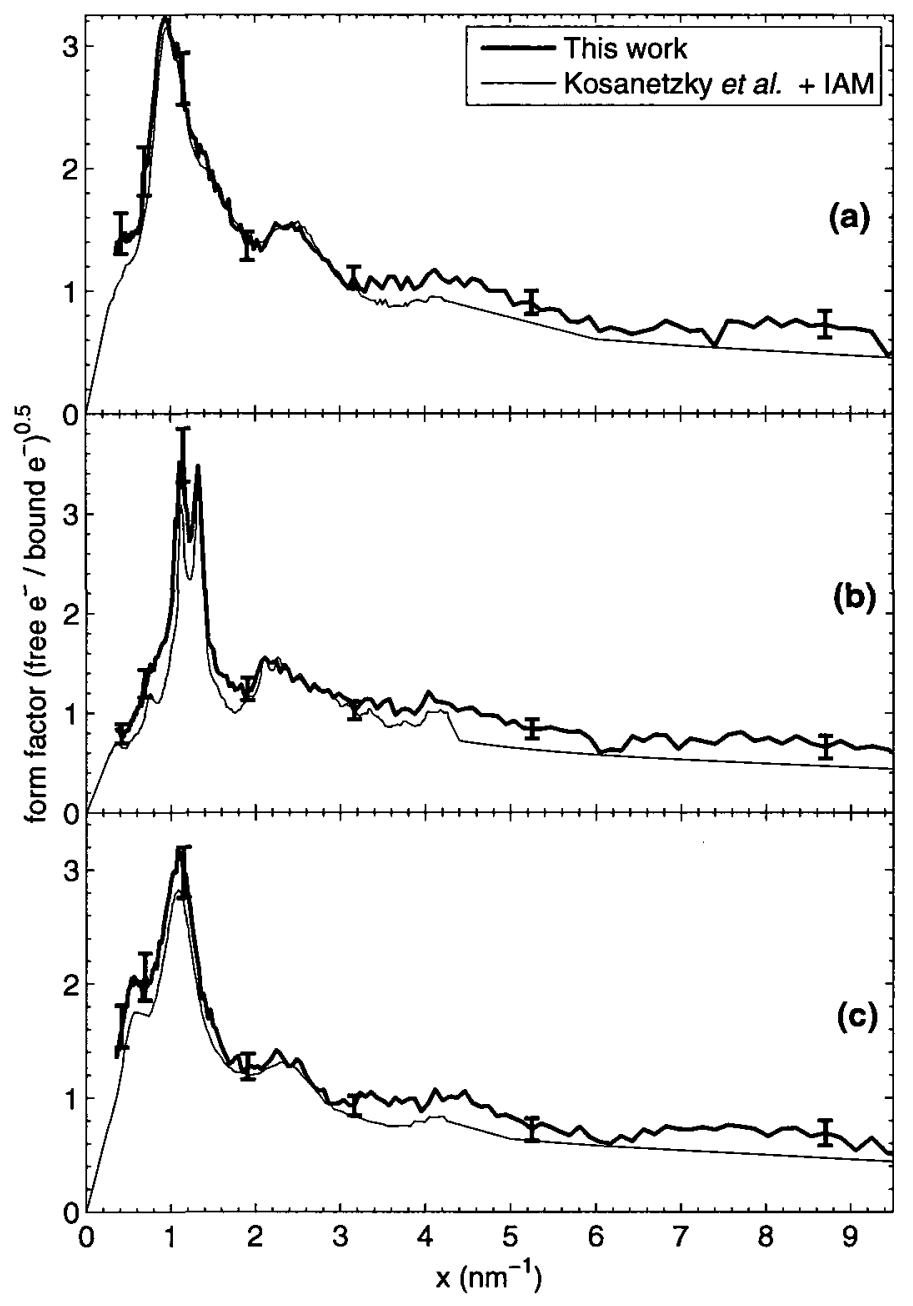

Figure 6.12: Combined form factor measurements of (a) polycarbonate, (b) nylon, and (c) polystyrene compared to published values[20]. Error bars are only shown for every 25 th point for clarity.

In order to make more quantitative comparisons with literature values, we computed the average absolute relative difference of the various measurements using Equation 4.1. The results are shown in Table 6.2. We also verified the repeatability of our water measurements through multiple measurements and found the results quite consistent $\left(D_{\mathrm{av}} \sim 0.05\right.$ for $0.5 \mathrm{~nm}^{-1}<x<9.5 \mathrm{~nm}^{-1}$ ). We verified that the presence of the target container was insignificant by comparing form factors measured for polyethylene both in the target container and on its own. There were no significant differences between the measurements. 
Table 6.2: Average absolute relative difference of the combined form factor measurements compared to published values[19, 20,21, 22].

\begin{tabular}{llll}
\hline Material & Comparison & $x$ range $\left(\mathrm{nm}^{-1}\right)$ & $D_{\mathrm{av}}$ \\
\hline \hline Water & Narten & $0.5-9.5$ & 0.097 \\
& Kosanetzky et al & $0.5-4.25$ & 0.085 \\
& Peplow \& Verghese & $0.5-9.5$ & 0.135 \\
& Hura et al & $0.5-9.5$ & 0.134 \\
Fat & Kosanetzky et al & $0.5-4.25$ & 0.254 \\
& Peplow \& Verghese & $0.5-9.5$ & 0.162 \\
Polyethylene & Kosanetzky et al & $0.5-4.25$ & 0.196 \\
Polycarbonate & Kosanetzky et al & $0.5-4.25$ & 0.080 \\
Nylon & Kosanetzky et al & $0.5-4.25$ & 0.189 \\
Polystyrene & Kosanetzky et al & $0.5-4.25$ & 0.128 \\
\hline
\end{tabular}

\subsection{Discussion}

The results of the energy-dispersive measurements are quite encouraging. The good agreement of the individual measurements with each other, as well as the fact that the measurements naturally approach the IAM value at large $x$ is very strong evidence of the accuracy of the energy-dispersive measurement. The strong correspondence with previously published diffractometer measurements is also promising.

The time required to set up and characterize the experiment is quite long. The alignment procedure is given in Appendix B. In addition, background spectra and fluence factors need to be measured for all configurations. It can take several days to a week to go through this from start to finish. After the system has been characterized, however, measurements are relatively quick. Each set of measurements requires a five minute, low current transmission spectrum to characterize the transmission aperture (aperture $\mathrm{G}$ in 
Figure 5.2), two, one minute transmission spectra and seven, five minute scatter spectra. Including the time for adjustment for each configuration, a complete measurement can be made in approximately one hour.

Our technique measures the form factor absolutely, with no scaling parameters. Diffractometer measurements are placed on an absolute scale by fitting the measured values to the IAM results at large $x$. However, it is not readily apparent exactly where the IAM region begins. If the range of $x$ is not large enough then the scaling can introduce a systematic shift in the measurement. This was certainly a problem in the results of Johns and Wismayer [23] and also possibly with some of the results of Kosanetzky et al [20]. Our energy-dispersive measurement avoids this problem entirely and allows an independent check that the experiment is configured correctly.

It is certainly possible to scale the energy-dispersive results to the IAM value at large $x$ once the region of validity of the IAM has been established. This could be achieved by scaling the results for the largest scattering angle to the IAM value at large $x$, then scaling the next largest results to the refined value in the overlap region, and so on until all of the scattering angles have been refined. Doing this may provide a more precise value of the geometrical parameters (primarily $V_{t}$ ) than can be achieved directly from the measured values, thus giving a more precise measurement of $F_{\mathrm{coh}}$. The series of cascaded scaling parameters however, would mean that the uncertainties would increase as the calculation progresses to smaller $x$ values. Additional study is required before determining whether this would be an improvement over the current method.

Even if it is advantageous to scale the measured values to the IAM, it should not be done lightly. The full calculation should be performed first and the self-consistency of the individual scatter results verified, as well as the region of validity of the IAM. Introducing an arbitrary scaling parameter would allow potential experimental configuration problems to be masked if it is used indiscriminately. The scaling should be used as a refinement of the measured parameters, not a replacement. 
Currently, there is very little benefit to be gained from longer exposures to improve the counting statistics as the uncertainty is dominated by the volume calculation. If scaling to the IAM value at large $x$ is found to be beneficial, the counting statistics in the scatter spectra may become more significant and longer exposures could provide more accurate results.

As was mentioned in Section 5.1.5, we have not used the detector correction algorithm of Section 2.3.3 for the HPGe spectrometer in this calculation. There are two reasons for this: First, the detector correction is most significant at the low end of the energy spectrum where the counts drop off toward zero. Our relatively crude detector characterization measurements tend to overestimate the correction in this region, affecting the ratio $\mathrm{d} N_{\mathrm{s}} / \mathrm{d} N_{\mathrm{t}}$ quite strongly. Because we can easily exclude a given energy range using the HPGe spectrometer, it was decided to forgo the detector correction and simply avoid the energy region where a correction is required. Second, the detector correction adds a significant amount of time for processing each spectrum. Since there are five spectra required for each of seven configurations, the extra time adds up quickly. Using the 1.8 $\mathrm{GHz}$ computer in our lab, calculation of a complete set of form factor measurements takes approximately 30 seconds without any detector correction and almost five minutes with the detector correction applied. We calculated the form factor for our water measurements both with the detector correction algorithm and without to verify that the results were similar and found that the effect was very small over the energy range allowed. See Figure 6.13.

We also verified that the exponential factors neglected in Equations 5.6 and 5.7 were indeed negligible by numerically evaluating the full integral in the worst case scenario: Low energy photons $(30 \mathrm{keV})$ with the largest scattering angle used in our experiment. We found that the exponential factors constituted less than a $0.5 \%$ effect on the volume calculation, justifying our decision to ignore them.

We modelled the apertures in the system as ideal, allowing no penetration. In reality, 


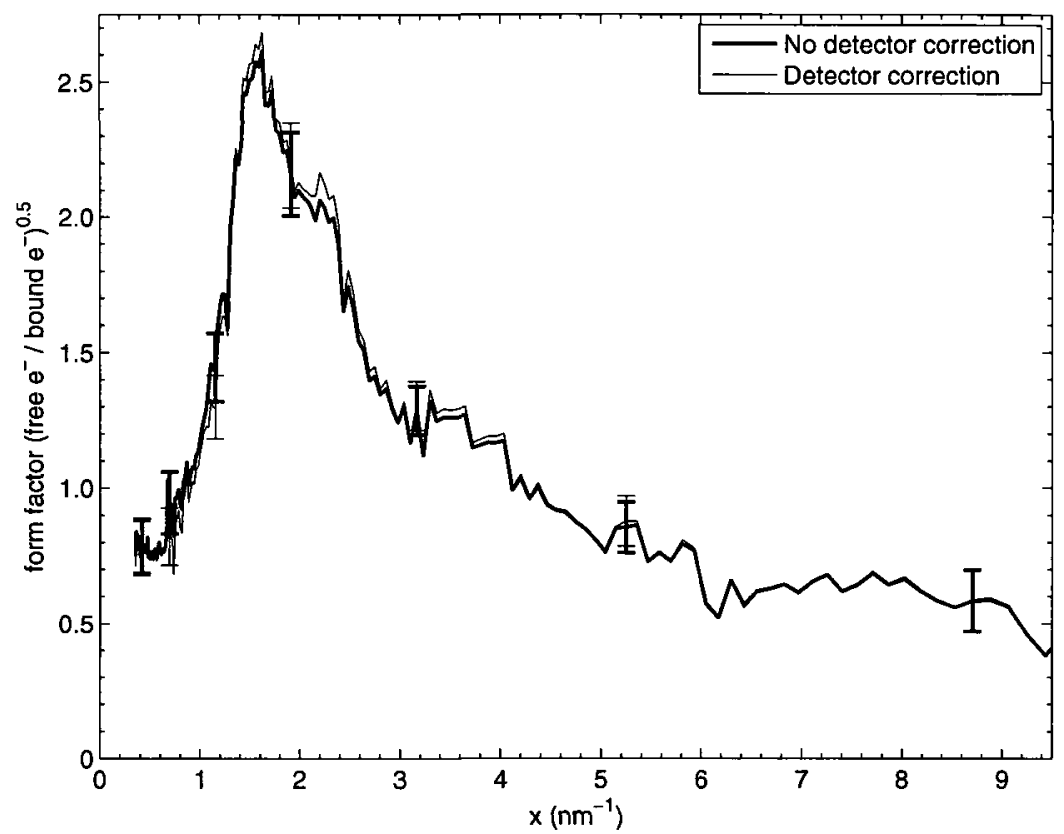

Figure 6.13: Form factor of water measured using the HPGe detector correction and without. Error bars are shown only for every 25 th point for clarity.

there would be a slight effect from penetration through the edges of the aperture, which would become more significant at larger $\theta$. This would introduce an additional energydependent effect to our measurements as more $\mathrm{x}$ rays penetrate through the edges of the apertures at higher energies. Since Figure 6.1 showed that there was no significant $\mathrm{Pb}$ $\mathrm{K}$-edge around $88 \mathrm{keV}$ in the spectra, we concluded that penetration through the apertures was insignificant.

The fairly long target used in these measurements was chosen to provide the best balance between scattering volume and target attenuation. As a first approximation, using Equation 5.6, the scatter countrate is proportional to the product of the target length and the transmission factor. Under this assumption, the optimal countrate will be found with a target length of $\mu^{-1}$ which for water gives a value between 3 and $6 \mathrm{~cm}$. For larger $\theta$ this approximation breaks down, however. The Monte Carlo calculation shows that for the largest scattering angle used in our experiment, the scattering volume is contained entirely within about half of the target length. A thinner target would therefore provide a better 
countrate at larger angles, at the expense of a reduced countrate for the smaller angles and larger uncertainties in the volume calculation. A thinner target would also make sample preparation easier, as a smaller volume of homogeneous tissue would be required.

The combination of the individual measurements into a single data set has advantages and disadvantages. Since each measured $x$ value (except at very high and low $x$ ) was measured at least twice, and in most cases three or more times, any variations that may be present tend to be reduced in the combined measurement. On the other hand, the difference in the $x$ resolution between different configurations means that, in some cases, the weighted average will not be appropriate. Our method of determining when to combine the results works fairly well but can sometimes cause small jumps in the combined measurements as adjacent points in $x$ space switch from a single measurement to a combined value. 


\section{CHAPTER 7}

\section{CONCLUSIONS}

To compare the angle-dispersive and energy-dispersive methods, Figures 7.1 and 7.2 show the results of each for water and fat. The $70 \mathrm{kV}$ sub-matrix results were chosen as representative samples of the angle-dispersive technique. The results agree quite well with each other although the peak values are slightly smaller for the sub-matrix calculation in both cases. Quantitative comparisons with all of the angle-dispersive measurements are shown in Table 7.1 by calculating the average absolute relative difference, $D_{\mathrm{av}}$ defined in equation 4.1.

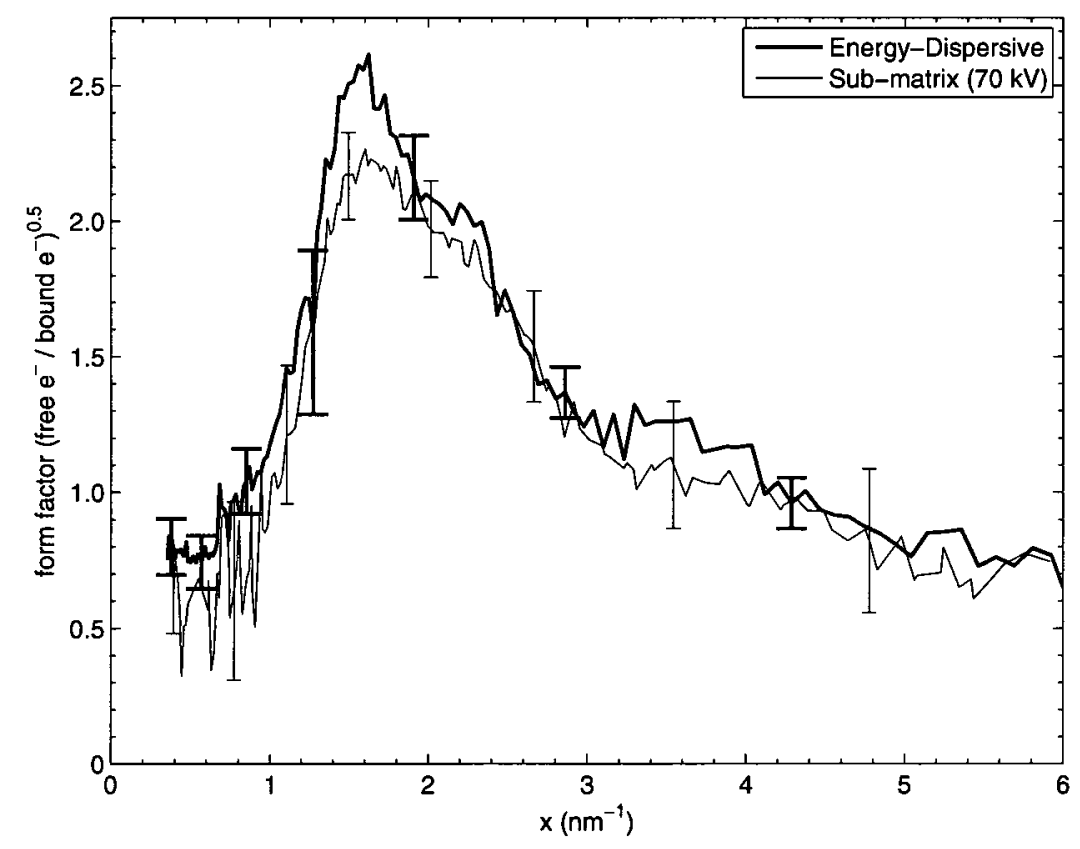

Figure 7.1: Comparison of energy-dispersive and sub-matrix form factor measurements for water. 


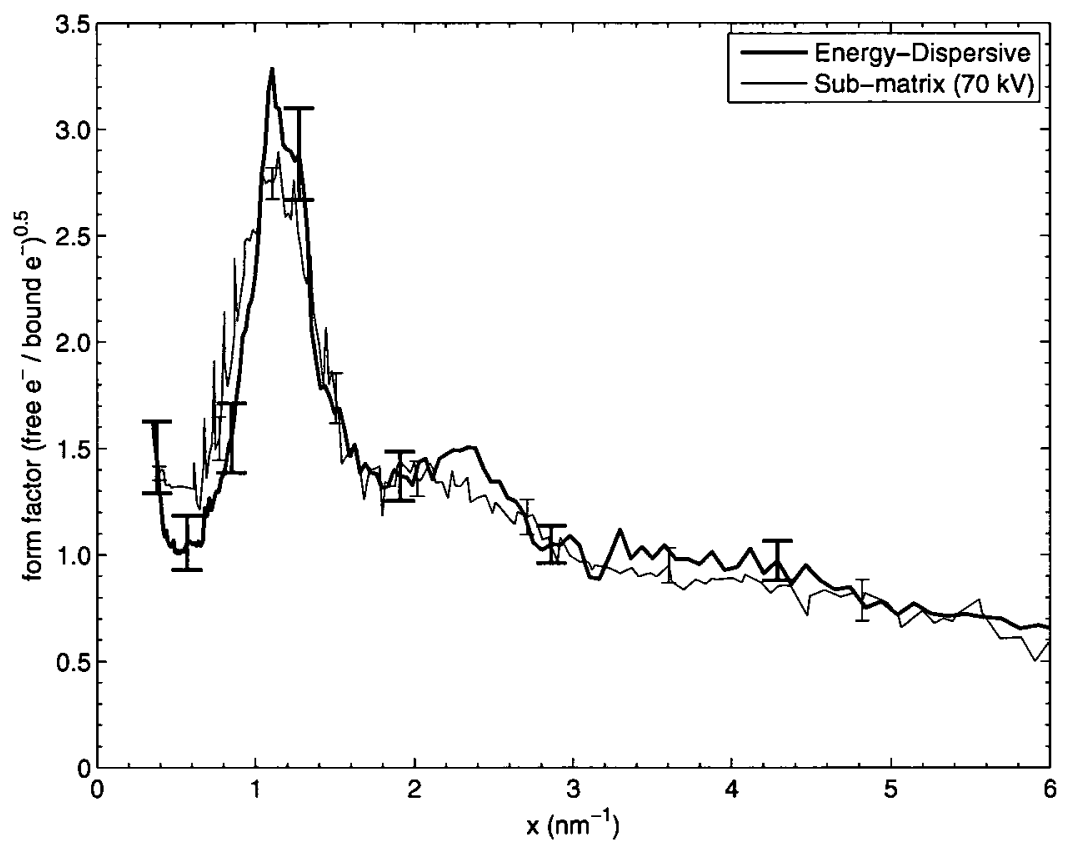

Figure 7.2: Comparison of energy-dispersive and sub-matrix form factor measurements for fat.

Table 7.1: Average absolute relative difference (from Equation 4.1) between the form factors measured with the energy-dispersive method in Chapter 6 and the angle-dispersive methods in Chapter 4. The range of $x$ values compared is between 0.5 and $4.0 \mathrm{~nm}^{-1}$. The angle-dispersive results were smoothed with a 5 point rectangular kernel before comparison.

\begin{tabular}{llll}
\hline Material & Angle-Dispersive Method & Potential $(\mathrm{kV})$ & $D_{\mathrm{av}}$ \\
\hline \hline Water & Sub-matrix & 50 & 0.201 \\
& & 70 & 0.126 \\
& & 92 & 0.167 \\
& Regularization & 50 & 0.282 \\
& & 70 & 0.190 \\
Fat & Sub-matrix & 92 & 0.175 \\
& & 50 & 0.336 \\
& & 70 & 0.117 \\
& Regularization & 50 & 0.321 \\
& & 70 & 0.102 \\
\hline
\end{tabular}


The energy-dispersive technique is more reliable than the angle-dispersive technique, giving better $x$ resolution, sharper peaks, and an overall cleaner signal. The ill-conditioning of the matrices in the angle-dispersive technique limits the accuracy of the measurement. Although the techniques developed in Chapter 3 improve the situation somewhat, the angle-dispersive technique does not have the accuracy required for precision form factor measurements.

\subsection{Discussion}

Previous form factor measurements of tissues have been measured from a crystallographic point of view, using essentially the same equipment and procedures. Crystallographic techniques are designed to measure the location of very sharply peaked form factors in $x$ space. This requires very high energy and angle resolution. In order to achieve the best angular resolution, they use a wide range of angles, which in turn leads to using relatively low-energy $\mathrm{x}$ rays to observe the relevant region in $x$ space. The use of low-energy $\mathrm{x}$ rays also means that the only practical scattering geometry is a reflection from the target surface. The low-energy $\mathrm{x}$ rays will not penetrate through any appreciable thickness of material.

The methods that I developed, on the other hand, were designed from the beginning with tissues in mind. I used high-energy $\mathrm{x}$ rays that easily penetrate through a significant thickness of material. This makes a transmission geometry possible, which provides a more consistent background subtraction and allows a direct measure of the x-ray tube fluence, removing the need for a scaling parameter. Both of the methods developed here sacrifice some $x$ resolution by being limited to relatively small scattering angles but this is not critical in the case of tissues with their broad peaks.

The limitation to small scattering angles is actually a benefit in the energy-dispersive measurement. In addition to the small angle simplifications discussed in Chapter 5, the 
small angles present in the system limit the effect that the slight polarization of the x-ray beam would cause in the scatter signal. For small scattering angles $\left(<20^{\circ}\right)$, the effect of polarization is negligible for all photon energies produced by the $\mathrm{x}$-ray tube, whereas for larger scattering angles, the stronger polarization at high energies can be much more significant.[47]

An alternative configuration that would also allow an energy-dispersive form factor measurement would be a stationary target with a detector that rotates around the centre of the target. This type of measurement has some advantages over my configuration. Chief among them is the knowledge that the input $\mathrm{x}$-ray fluence is consistent for all scattering angles, therefore removing the need to measure the overall fluence ratio. On the other hand, alignment would be more difficult with the large detector that I used.

The accuracy of both the energy-dispersive and angle-dispersive methods is poor at small $x$ values. This is partly a result of having to measure at very small angles, quite close to the strong primary beam. Improved accuracy could be obtained by using a lower energy $\mathrm{x}$-ray source. Using my current configuration with energies between 15 and $35 \mathrm{keV}$ from a Mo anode $\mathrm{x}$-ray tube in place of the $\mathrm{W}$ anode would allow form factor measurements in the range $0.18 \mathrm{~nm}^{-1}<x<3.75 \mathrm{~nm}^{-1}$, spreading the low $x$ measurements to larger values of $\theta$. As the energy-dispersive method does not require scaling to the IAM value, there is no requirement to measure to larger $x$ values. On the other hand, a thinner target would be required in order to allow significant penetration through the sample. This could negate some of the advantages of the lower energy $\mathrm{x}$ rays. More detailed calculations would need to be done to examine this possibility.

I showed that improvements in accuracy with the energy-dispersive method would require tighter geometrical tolerances to improve the precision in the scattering volume. The apertures I constructed were all manually machined, allowing for some slight variations. Computer controlled machining would be more precise, giving better results. A harder material would also be an improvement. The $\mathrm{Pb}$ sheets dimple slightly around the edges 
when punched, leading to slightly rounded aperture edges, as shown in Figure 7.3. This could explain the lack of any significant $\mathrm{Pb}$ penetration through the edges of the apertures and also means that the apertures may be very slightly larger than measured.

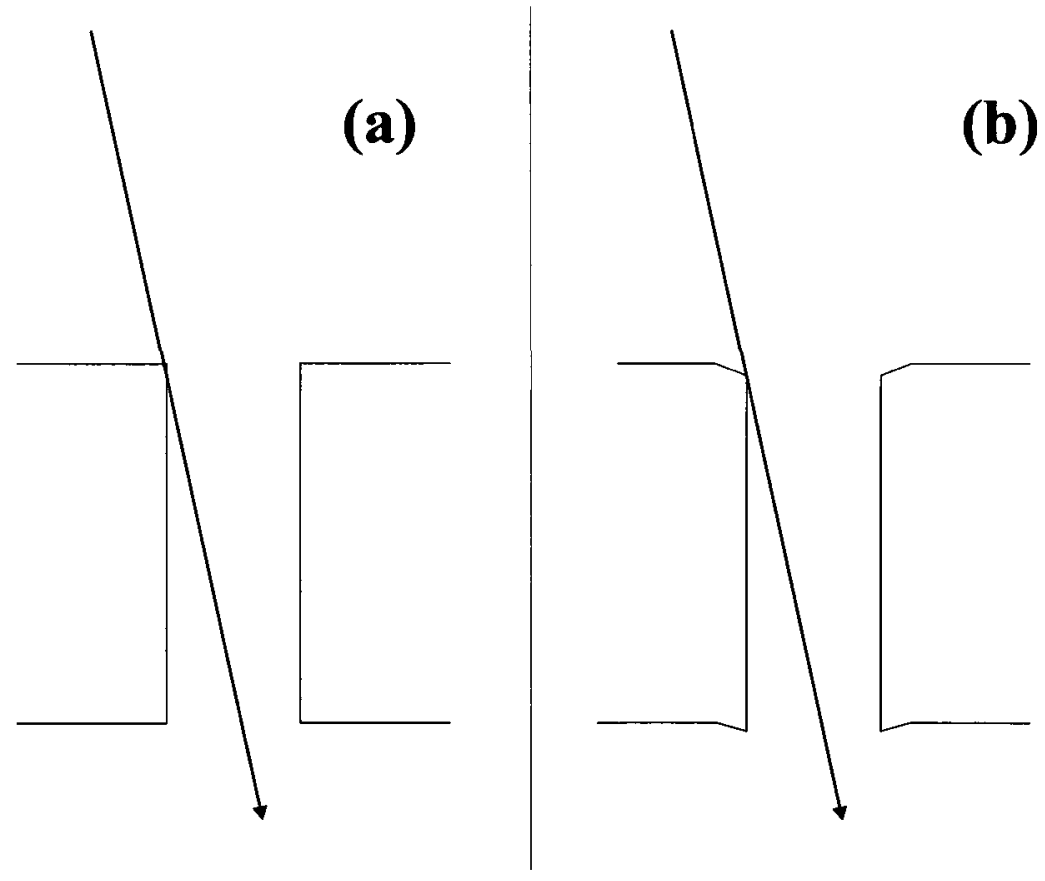

Figure 7.3: Sketch of (a) ideal aperture modelled for volume calculation and (b) experimental aperture. Not drawn to scale.

I also showed that corrections for the detector response and for pileup do not make a significant contribution to the measurement. A more reliable method of spectrum correction for the detector response and for pileup effects would slightly extend the range of $x$ available. Monte Carlo calculations of the detector response could be used to develop a more precise model of the detector response. Methods have also been developed to correct spectra for pileup [48]. Before applying these techniques to the form factor calculation, however, they need to be thoroughly characterized and tested. The effect of these corrections will be most significant at the edges of the spectrum where the countrate drops to near zero, making the measurements quite sensitive. Additional sensitivity is caused as a result of the fact that the measurement requires calculation of the ratio $\mathrm{d} N_{\mathrm{s}} / \mathrm{d} N_{\mathrm{t}}$ as both values approach zero. Slight inaccuracies in the correction could lead to erroneous results. 
My measured results for both angle-dispersive and energy-dispersive calculations rely on the tabulated values of $F_{\text {inc }}$ being correct. However, this formulation is not exact. It is estimated to be accurate to better than $5 \%$ for small angle scattering and low $\mathrm{Z}$ materials[49]. More detailed formulations have been developed that show the incoherent scattering is not strictly a function of $x$, however there are very limited data to determine which formulation is correct[49]. My angle-dispersive and energy-dispersive formulations could be modified to include a different incoherent contribution, if it is shown to be necessary. The agreement of my results with the IAM value suggests that the effect is not significant for the current levels of accuracy.

\subsection{Future Work}

I am currently using the energy-dispersive method to measure form factors of additional tissues and phantom materials with the aim of developing a comprehensive library. Measurements of tissue form factors will, for the most part, follow the procedure defined for fat measurements. For tissues that are not easily found in large enough pieces, a number of smaller pieces will have to be placed in the container together. Multiple scattering could play a more significant role in the case of bone, since it has a much higher attenuation coefficient. It may therefore be necessary to use a thinner target for measurement of bone.

Previous measurements of white and grey brain matter have shown significant differences in the form factors in the vicinity of $x=1 \mathrm{~nm}^{-1}$ (see Reference [20]), suggesting that scatter imaging may prove very useful in this area. It will be very interesting to see if these results are confirmed by the energy-dispersive measurements.

Accurate knowledge of the form factors will allow scattering cross-sections for these materials to be determined for any experimental configuration. This will aid in the development of the field of scatter imaging by allowing accurate scattering models to be developed and tested. It will enable a much better understanding of where scatter imaging may 
give improved image quality. These models can also be used to improve primary beam imaging by determining what type of systems to use to minimize the effect of scattering.

There has also been interest recently in using $x$-ray scattering in the field of security and baggage screening. It may be possible to more easily identify explosives using their scattering properties than with primary beam imaging[50]. Measuring the form factors of these types of materials would certainly be possible with our equipment and could aid the development of this field as well.

\subsection{Final Thoughts}

In this thesis, I have developed two new methods of measuring coherent scattering form factors of tissues that avoid some of the problems associated with crystallographic measurements. The angle-dispersive measurements of Chapters 3 and 4 can be implemented with standard clinical equipment but are noisy because of the ill-conditioning of the problem. I have shown that mathematical methods to extract the form factor do exist, even in the presence of the ill-conditioning, although these methods are not always reliable and can give spurious results in some cases.

I applied these methods experimentally and showed that low resolution measurements of tissue form factors are possible with practical, clinical equipment, although the accuracy of my measurements was limited by output variations in the signal strength. The experimental measurements were somewhat degraded as compared to the predicted values from simulations. This could have been due to limitations of the model or the effect of the large uncertainties on an already difficult problem.

The energy-dispersive technique that I developed in this thesis has been thoroughly characterized and shown to accurately measure x-ray coherent scattering form factors of amorphous materials. The form factors of water, fat and several plastics have been measured between $0.5 \mathrm{~nm}^{-1}$ and $9.5 \mathrm{~nm}^{-1}$. The $x$ resolution was shown to be betweer 2 and 
$10 \%$, larger than diffractometer measurements but more than sufficient for measurements with amorphous materials. The accuracy of the form factor measurements was shown to be around $10 \%$. These measurements are the first of their kind, measuring over a large momentum transfer range, well into the IAM region, without the need for an arbitrary scaling constant. This method has some advantages over diffractometer measurements when measuring scattering from amorphous materials because the transmission geometry allows a more reliable background subtraction and because there is no monochromator producing angle-dependent efficiency effects. Ongoing tissue measurements using this method should resolve some of the uncertainties in the current state of knowledge of x-ray scattering. 


\section{APPENDIX A}

\section{FORM FACTOR RESULTS}

Data tables containing the measured form factors for the materials studied in Chapter 6 are included here. These tables allow the scattering properties of the measured materials to be calculated for any experimental configuration. The values for the incoherent scattering form factor $F_{\text {inc }}$ were tabulated based on data from Reference [17]. In order to accurately compute the scattering cross-section from these data using Equation 2.19, the values for the electron density and $F_{\text {inc }}$ must be the ones given in these tables. The measured values

of $F_{\mathrm{coh}}$ are based on these values. Using a different electron density or values for $F_{\mathrm{inc}}$ will give inconsistent results. Not every measured point is included in these tables to save space. 
Table A.1: Measured form factor of water using the energy-dispersive technique. The units of $F_{\text {coh }}$ and its standard deviation (s.d.) are (free $\mathrm{e}^{-} /$bound $\left.\mathrm{e}^{-}\right)^{0.5}$ and the units of $F_{\text {inc }}$ are (free $\mathrm{e}^{-} /$bound $\mathrm{e}^{-}$). The electron density is $\rho_{\mathrm{e}}=3.3348 \times 10^{23} \mathrm{~cm}^{-3}$.

\begin{tabular}{llll|llll}
\hline $\begin{array}{l}\mathrm{x} \\
\left(\mathrm{nm}^{-1}\right)\end{array}$ & $F_{\text {coh }}$ & s.d. & $F_{\text {inc }}$ & $\begin{array}{l}\mathrm{x} \\
\left(\mathrm{nm}^{-1}\right)\end{array}$ & $F_{\text {coh }}$ & s.d. & $F_{\text {inc }}$ \\
\hline \hline $3.71 \mathrm{e}-001$ & 0.82 & 0.11 & 0.027 & $2.29 \mathrm{e}+000$ & 1.98 & 0.11 & 0.497 \\
$3.94 \mathrm{e}-001$ & 0.77 & 0.10 & 0.030 & $2.43 \mathrm{e}+000$ & 1.65 & 0.17 & 0.525 \\
$4.19 \mathrm{e}-001$ & 0.79 & 0.10 & 0.034 & $2.59 \mathrm{e}+000$ & 1.54 & 0.10 & 0.552 \\
$4.45 \mathrm{e}-001$ & 0.79 & 0.10 & 0.038 & $2.75 \mathrm{e}+000$ & 1.41 & 0.10 & 0.579 \\
$4.73 \mathrm{e}-001$ & 0.82 & 0.10 & 0.043 & $2.92 \mathrm{e}+000$ & 1.29 & 0.09 & 0.605 \\
$5.02 \mathrm{e}-001$ & 0.74 & 0.10 & 0.048 & $3.10 \mathrm{e}+000$ & 1.17 & 0.09 & 0.630 \\
$5.34 \mathrm{e}-001$ & 0.74 & 0.10 & 0.054 & $3.30 \mathrm{e}+000$ & 1.32 & 0.11 & 0.654 \\
$5.67 \mathrm{e}-001$ & 0.74 & 0.10 & 0.061 & $3.50 \mathrm{e}+000$ & 1.26 & 0.10 & 0.677 \\
$6.03 \mathrm{e}-001$ & 0.77 & 0.10 & 0.068 & $3.72 \mathrm{e}+000$ & 1.15 & 0.10 & 0.698 \\
$6.40 \mathrm{e}-001$ & 0.77 & 0.10 & 0.076 & $3.95 \mathrm{e}+000$ & 1.17 & 0.10 & 0.719 \\
$6.80 \mathrm{e}-001$ & 1.03 & 0.12 & 0.085 & $4.20 \mathrm{e}+000$ & 1.04 & 0.09 & 0.737 \\
$7.23 \mathrm{e}-001$ & 0.92 & 0.11 & 0.095 & $4.46 \mathrm{e}+000$ & 0.94 & 0.09 & 0.754 \\
$7.68 \mathrm{e}-001$ & 0.97 & 0.11 & 0.106 & $4.74 \mathrm{e}+000$ & 0.88 & 0.09 & 0.769 \\
$8.16 \mathrm{e}-001$ & 0.92 & 0.11 & 0.118 & $5.04 \mathrm{e}+000$ & 0.76 & 0.09 & 0.784 \\
$8.67 \mathrm{e}-001$ & 1.09 & 0.12 & 0.131 & $5.36 \mathrm{e}+000$ & 0.86 & 0.09 & 0.796 \\
$9.22 \mathrm{e}-001$ & 1.08 & 0.12 & 0.145 & $5.69 \mathrm{e}+000$ & 0.73 & 0.10 & 0.808 \\
$9.79 \mathrm{e}-001$ & 1.13 & 0.13 & 0.161 & $6.05 \mathrm{e}+000$ & 0.57 & 0.10 & 0.819 \\
$1.04 \mathrm{e}+000$ & 1.26 & 0.14 & 0.177 & $6.43 \mathrm{e}+000$ & 0.57 & 0.11 & 0.828 \\
$1.11 \mathrm{e}+000$ & 1.46 & 0.13 & 0.196 & $6.83 \mathrm{e}+000$ & 0.64 & 0.10 & 0.837 \\
$1.17 \mathrm{e}+000$ & 1.60 & 0.13 & 0.215 & $7.26 \mathrm{e}+000$ & 0.68 & 0.10 & 0.846 \\
$1.25 \mathrm{e}+000$ & 1.71 & 0.14 & 0.236 & $7.71 \mathrm{e}+000$ & 0.69 & 0.11 & 0.855 \\
$1.33 \mathrm{e}+000$ & 2.05 & 0.16 & 0.258 & $8.19 \mathrm{e}+000$ & 0.62 & 0.11 & 0.863 \\
$1.41 \mathrm{e}+000$ & 2.27 & 0.16 & 0.281 & $8.70 \mathrm{e}+000$ & 0.58 & 0.11 & 0.871 \\
$1.50 \mathrm{e}+000$ & 2.51 & 0.18 & 0.306 & $9.25 \mathrm{e}+000$ & 0.46 & 0.13 & 0.879 \\
$1.59 \mathrm{e}+000$ & 2.56 & 0.18 & 0.331 & $9.83 \mathrm{e}+000$ & 0.45 & 0.14 & 0.888 \\
$1.69 \mathrm{e}+000$ & 2.41 & 0.17 & 0.357 & & & & \\
$1.80 \mathrm{e}+000$ & 2.31 & 0.16 & 0.385 & & & & \\
$1.91 \mathrm{e}+000$ & 2.16 & 0.15 & 0.412 & & & & \\
$2.03 \mathrm{e}+000$ & 2.08 & 0.15 & 0.440 & & & & \\
$2.16 \mathrm{e}+000$ & 1.99 & 0.12 & 0.468 & & & & \\
\hline & & & & & & &
\end{tabular}


Table A.2: Measured form factor of fat using the energy-dispersive technique. The units of $F_{\text {coh }}$ and its standard deviation (s.d.) are (free $\mathrm{e}^{-} /$bound $\left.\mathrm{e}^{-}\right)^{0.5}$ and the units of $F_{\text {inc }}$ are (free $\mathrm{e}^{-/}$bound $\mathrm{e}^{-}$). The electron density is $\rho_{\mathrm{e}}=3.09 \times 10^{23} \mathrm{~cm}^{-3}$.

\begin{tabular}{llll|llll}
\hline $\begin{array}{l}\mathrm{x} \\
\left(\mathrm{nm}^{-1}\right)\end{array}$ & $F_{\text {coh }}$ & s.d. & $F_{\text {inc }}$ & $\begin{array}{l}\mathrm{x} \\
\left(\mathrm{nm}^{-1}\right)\end{array}$ & $F_{\text {coh }}$ & s.d. & $F_{\text {inc }}$ \\
\hline \hline $3.71 \mathrm{e}-001$ & 1.50 & 0.17 & 0.033 & $2.29 \mathrm{e}+000$ & 1.49 & 0.10 & 0.550 \\
$3.94 \mathrm{e}-001$ & 1.30 & 0.15 & 0.037 & $2.43 \mathrm{e}+000$ & 1.41 & 0.09 & 0.577 \\
$4.19 \mathrm{e}-001$ & 1.12 & 0.13 & 0.042 & $2.59 \mathrm{e}+000$ & 1.27 & 0.09 & 0.603 \\
$4.45 \mathrm{e}-001$ & 1.07 & 0.13 & 0.047 & $2.75 \mathrm{e}+000$ & 1.06 & 0.09 & 0.628 \\
$4.73 \mathrm{e}-001$ & 1.04 & 0.12 & 0.053 & $2.92 \mathrm{e}+000$ & 1.05 & 0.09 & 0.652 \\
$5.02 \mathrm{e}-001$ & 1.01 & 0.12 & 0.059 & $3.10 \mathrm{e}+000$ & 0.90 & 0.08 & 0.673 \\
$5.34 \mathrm{e}-001$ & 1.02 & 0.12 & 0.066 & $3.30 \mathrm{e}+000$ & 1.12 & 0.10 & 0.693 \\
$5.67 \mathrm{e}-001$ & 1.06 & 0.13 & 0.074 & $3.50 \mathrm{e}+000$ & 0.98 & 0.10 & 0.712 \\
$6.03 \mathrm{e}-001$ & 1.05 & 0.13 & 0.083 & $3.72 \mathrm{e}+000$ & 0.98 & 0.09 & 0.730 \\
$6.40 \mathrm{e}-001$ & 1.06 & 0.13 & 0.093 & $3.95 \mathrm{e}+000$ & 0.93 & 0.09 & 0.746 \\
$6.80 \mathrm{e}-001$ & 1.22 & 0.13 & 0.103 & $4.20 \mathrm{e}+000$ & 0.91 & 0.09 & 0.760 \\
$7.23 \mathrm{e}-001$ & 1.21 & 0.13 & 0.115 & $4.46 \mathrm{e}+000$ & 0.95 & 0.09 & 0.773 \\
$7.68 \mathrm{e}-001$ & 1.29 & 0.14 & 0.128 & $4.74 \mathrm{e}+000$ & 0.85 & 0.09 & 0.785 \\
$8.16 \mathrm{e}-001$ & 1.43 & 0.15 & 0.142 & $5.04 \mathrm{e}+000$ & 0.72 & 0.10 & 0.796 \\
$8.67 \mathrm{e}-001$ & 1.65 & 0.17 & 0.157 & $5.36 \mathrm{e}+000$ & 0.71 & 0.10 & 0.807 \\
$9.22 \mathrm{e}-001$ & 2.02 & 0.21 & 0.174 & $5.69 \mathrm{e}+000$ & 0.70 & 0.10 & 0.817 \\
$9.79 \mathrm{e}-001$ & 2.20 & 0.22 & 0.192 & $6.05 \mathrm{e}+000$ & 0.65 & 0.10 & 0.826 \\
$1.04 \mathrm{e}+000$ & 2.79 & 0.28 & 0.211 & $6.43 \mathrm{e}+000$ & 0.62 & 0.10 & 0.836 \\
$1.11 \mathrm{e}+000$ & 3.28 & 0.24 & 0.231 & $6.83 \mathrm{e}+000$ & 0.65 & 0.10 & 0.846 \\
$1.17 \mathrm{e}+000$ & 2.92 & 0.22 & 0.253 & $7.26 \mathrm{e}+000$ & 0.60 & 0.10 & 0.856 \\
$1.25 \mathrm{e}+000$ & 2.85 & 0.21 & 0.276 & $7.71 \mathrm{e}+000$ & 0.55 & 0.12 & 0.865 \\
$1.33 \mathrm{e}+000$ & 2.52 & 0.19 & 0.301 & $8.19 \mathrm{e}+000$ & 0.60 & 0.11 & 0.875 \\
$1.41 \mathrm{e}+000$ & 1.78 & 0.14 & 0.327 & $8.70 \mathrm{e}+000$ & 0.54 & 0.12 & 0.885 \\
$1.50 \mathrm{e}+000$ & 1.67 & 0.13 & 0.353 & $9.25 \mathrm{e}+000$ & 0.60 & 0.11 & 0.895 \\
$1.59 \mathrm{e}+000$ & 1.46 & 0.12 & 0.381 & $9.83 \mathrm{e}+000$ & 0.34 & 0.17 & 0.905 \\
$1.69 \mathrm{e}+000$ & 1.43 & 0.12 & 0.408 & $1.04 \mathrm{e}+001$ & 0.37 & 0.16 & 0.915 \\
$1.80 \mathrm{e}+000$ & 1.31 & 0.11 & 0.437 & & & & \\
$1.91 \mathrm{e}+000$ & 1.37 & 0.12 & 0.465 & & & & \\
$2.03 \mathrm{e}+000$ & 1.44 & 0.12 & 0.494 & & & & \\
$2.16 \mathrm{e}+000$ & 1.45 & 0.09 & 0.522 & & & & \\
\hline
\end{tabular}


Table A.3: Measured form factor of polyethylene using the energydispersive technique. The units of $F_{\text {coh }}$ and its standard deviation (s.d.) are (free $\mathrm{e}^{-} /$bound $\left.\mathrm{e}^{-}\right)^{0.5}$ and the units of $F_{\text {inc }}$ are (free $\mathrm{e}^{-} /$bound $\mathrm{e}^{-}$). The electron density is $\rho_{\mathrm{e}}=3.255 \times 10^{23} \mathrm{~cm}^{-3}$.

\begin{tabular}{|c|c|c|c|c|c|c|c|}
\hline $\begin{array}{l}\mathrm{x} \\
\left(\mathrm{nm}^{-1}\right) \\
\end{array}$ & $F_{\text {coh }}$ & s.d. & $F_{\text {inc }}$ & $\begin{array}{l}\mathrm{x} \\
\left(\mathrm{nm}^{-1}\right) \\
\end{array}$ & $F_{\mathrm{coh}}$ & s.d. & $F_{\text {inc }}$ \\
\hline $3.71 \mathrm{e}-001$ & 0.65 & 0.09 & 0.036 & $2.29 \mathrm{e}+000$ & 1.45 & $\overline{0.09}$ & $\overline{581}$ \\
\hline $3.94 \mathrm{e}-001$ & 0.61 & 0.08 & 0.041 & & 1.10 & 0.08 & 0.6 \\
\hline & 0.61 & 0.08 & 0.046 & & 1.07 & 0.08 & 0.633 \\
\hline & 0.55 & 0.08 & 0.052 & & 0.81 & 0.09 & 0.657 \\
\hline & 0.57 & 0.08 & 0.058 & & 1.15 & 0.09 & 0.680 \\
\hline 5.02 & 0.60 & 0.08 & 0.065 & & 0.86 & 0.09 & 0.100 \\
\hline 5.3 & 0.57 & 0.08 & 0.073 & & 99 & 0.10 & 0.718 \\
\hline 5. & 0.61 & 0.09 & 0.081 & & 0.91 & 0.10 & 0.736 \\
\hline 6. & 0.65 & 0.09 & 0.091 & & 0.80 & 0.10 & 0.752 \\
\hline 6. & 8 & 0.09 & 0.101 & & 0.98 & 0.10 & 0.766 \\
\hline 6.8 & 79 & 0.10 & 0.113 & 0 & 0.82 & 0.10 & 0.778 \\
\hline 7.2 & 0.84 & 0.10 & 0.126 & 4.4 & 0.93 & 0.10 & 0.789 \\
\hline 7.68 & 0.84 & 0.10 & 0.140 & 4.7 & 0.72 & 0.10 & 0.800 \\
\hline 8.16 & 0.96 & 0.11 & 0.155 & 5.0 & 0.72 & 0.10 & 0.810 \\
\hline $8.6^{\prime}$ & 1.06 & 0.12 & 0.171 & 5.3 & 0.64 & 0.10 & 0.819 \\
\hline 9.22 & 1.25 & 0.14 & 0.189 & 5.6 & 0.68 & 0.10 & 0.828 \\
\hline 9.79 & 1.49 & 0.16 & 0.209 & 6.0 & 0.64 & 0.10 & 0.837 \\
\hline-000 & 1.71 & 0.18 & 0.229 & 6.43 & 0.57 & 0.11 & 0.846 \\
\hline+000 & 2.00 & 0.16 & 0.251 & 6.83 & 0.52 & 0.12 & 0.856 \\
\hline $1.17 \mathrm{e}+000$ & 4.29 & 0.32 & 0.274 & 7.26 & 0.58 & 0.11 & 0.865 \\
\hline $1.25 \mathrm{e}+000$ & 3.35 & 0.25 & 0.298 & $7.71 \mathrm{e}$ & 0.64 & 0.11 & 0.875 \\
\hline+000 & 3.34 & 0.25 & 0.325 & 8.19 & 0.58 & 0.12 & 0.885 \\
\hline $1.41 \mathrm{e}+000$ & 1.13 & 0.11 & 0.352 & 8.70 & 0.59 & 0.12 & 0.895 \\
\hline $1.50 \mathrm{e}+000$ & 0.95 & 0.10 & 0.380 & 9.25 & 0.58 & 0.12 & 0.906 \\
\hline 000 & .81 & 0.10 & 0.408 & 9.83 & 0.57 & 0.12 & 0.916 \\
\hline 1.69 & .94 & 0.10 & 0.437 & $1.04 \mathrm{e}+001$ & 0.46 & 0.15 & 0.926 \\
\hline 1.80 & 0.65 & 0.10 & 0.4 & & & & \\
\hline & 8 & 0 & 0 & & & & \\
\hline & & 0.1 & 0 & & & & \\
\hline $2.16 \mathrm{e}+000$ & 1.28 & 0.09 & 0.5 & & & & \\
\hline
\end{tabular}


Table A.4: Measured form factor of polycarbonate using the energydispersive technique. The units of $F_{\text {coh }}$ and its standard deviation (s.d.) are (free $\mathrm{e}^{-} /$bound $\left.\mathrm{e}^{-}\right)^{0.5}$ and the units of $F_{\text {inc }}$ are (free $\mathrm{e}^{-/}$bound $\mathrm{e}^{-}$). The electron density is $\rho_{\mathrm{e}}=3.71 \times 10^{23} \mathrm{~cm}^{-3}$.

\begin{tabular}{llll|llll}
\hline $\begin{array}{l}\mathrm{X} \\
\left(\mathrm{nm}^{-1}\right)\end{array}$ & $F_{\text {coh }}$ & s.d. & $F_{\text {inc }}$ & $\begin{array}{l}\mathrm{x} \\
\left(\mathrm{nm}^{-1}\right)\end{array}$ & $F_{\text {coh }}$ & s.d. & $F_{\text {inc }}$ \\
\hline \hline $3.71 \mathrm{e}-001$ & 1.40 & 0.16 & 0.030 & $2.29 \mathrm{e}+000$ & 1.52 & 0.09 & 0.516 \\
$3.94 \mathrm{e}-001$ & 1.36 & 0.15 & 0.034 & $2.43 \mathrm{e}+000$ & 1.55 & 0.09 & 0.543 \\
$4.19 \mathrm{e}-001$ & 1.47 & 0.17 & 0.038 & $2.59 \mathrm{e}+000$ & 1.42 & 0.09 & 0.569 \\
$4.45 \mathrm{e}-001$ & 1.46 & 0.16 & 0.043 & $2.75 \mathrm{e}+000$ & 1.31 & 0.10 & 0.594 \\
$4.73 \mathrm{e}-001$ & 1.47 & 0.17 & 0.048 & $2.92 \mathrm{e}+000$ & 1.17 & 0.09 & 0.619 \\
$5.02 \mathrm{e}-001$ & 1.44 & 0.16 & 0.054 & $3.10 \mathrm{e}+000$ & 1.02 & 0.09 & 0.641 \\
$5.34 \mathrm{e}-001$ & 1.45 & 0.16 & 0.061 & $3.30 \mathrm{e}+000$ & 0.99 & 0.10 & 0.661 \\
$5.67 \mathrm{e}-001$ & 1.46 & 0.17 & 0.068 & $3.50 \mathrm{e}+000$ & 1.02 & 0.10 & 0.681 \\
$6.03 \mathrm{e}-001$ & 1.50 & 0.17 & 0.076 & $3.72 \mathrm{e}+000$ & 1.01 & 0.10 & 0.700 \\
$6.40 \mathrm{e}-001$ & 1.49 & 0.17 & 0.085 & $3.95 \mathrm{e}+000$ & 1.04 & 0.10 & 0.717 \\
$6.80 \mathrm{e}-001$ & 1.96 & 0.20 & 0.094 & $4.20 \mathrm{e}+000$ & 1.10 & 0.10 & 0.731 \\
$7.23 \mathrm{e}-001$ & 2.02 & 0.20 & 0.105 & $4.46 \mathrm{e}+000$ & 1.04 & 0.10 & 0.745 \\
$7.68 \mathrm{e}-001$ & 2.29 & 0.23 & 0.117 & $4.74 \mathrm{e}+000$ & 1.00 & 0.09 & 0.758 \\
$8.16 \mathrm{e}-001$ & 2.61 & 0.26 & 0.130 & $5.04 \mathrm{e}+000$ & 0.88 & 0.09 & 0.771 \\
$8.67 \mathrm{e}-001$ & 3.02 & 0.29 & 0.144 & $5.36 \mathrm{e}+000$ & 0.84 & 0.10 & 0.782 \\
$9.22 \mathrm{e}-001$ & 3.20 & 0.31 & 0.159 & $5.69 \mathrm{e}+000$ & 0.75 & 0.10 & 0.793 \\
$9.79 \mathrm{e}-001$ & 3.18 & 0.31 & 0.176 & $6.05 \mathrm{e}+000$ & 0.67 & 0.09 & 0.804 \\
$1.04 \mathrm{e}+000$ & 2.98 & 0.29 & 0.193 & $6.43 \mathrm{e}+000$ & 0.65 & 0.10 & 0.815 \\
$1.11 \mathrm{e}+000$ & 2.94 & 0.22 & 0.212 & $6.83 \mathrm{e}+000$ & 0.75 & 0.10 & 0.826 \\
$1.17 \mathrm{e}+000$ & 2.48 & 0.19 & 0.232 & $7.26 \mathrm{e}+000$ & 0.68 & 0.10 & 0.837 \\
$1.25 \mathrm{e}+000$ & 2.29 & 0.18 & 0.254 & $7.71 \mathrm{e}+000$ & 0.75 & 0.11 & 0.848 \\
$1.33 \mathrm{e}+000$ & 2.08 & 0.16 & 0.277 & $8.19 \mathrm{e}+000$ & 0.71 & 0.11 & 0.860 \\
$1.41 \mathrm{e}+000$ & 2.13 & 0.16 & 0.301 & $8.70 \mathrm{e}+000$ & 0.73 & 0.11 & 0.871 \\
$1.50 \mathrm{e}+000$ & 1.97 & 0.15 & 0.326 & $9.25 \mathrm{e}+000$ & 0.67 & 0.11 & 0.883 \\
$1.59 \mathrm{e}+000$ & 1.82 & 0.14 & 0.352 & $9.83 \mathrm{e}+000$ & 0.42 & 0.15 & 0.894 \\
$1.69 \mathrm{e}+000$ & 1.74 & 0.13 & 0.378 & & & & \\
$1.80 \mathrm{e}+000$ & 1.50 & 0.12 & 0.405 & & & & \\
$1.91 \mathrm{e}+000$ & 1.37 & 0.12 & 0.433 & & & & \\
$2.03 \mathrm{e}+000$ & 1.41 & 0.12 & 0.460 & & & & \\
$2.16 \mathrm{e}+000$ & 1.43 & 0.09 & 0.488 & & & & \\
\hline & & & & & & &
\end{tabular}


Table A.5: Measured form factor of polystyrene using the energy-dispersive technique. The units of $F_{\text {coh }}$ and its standard deviation (s.d.) are (free $\mathrm{e}^{-1}$ bound $\left.\mathrm{e}^{-}\right)^{0.5}$ and the units of $F_{\text {inc }}$ are (free $\mathrm{e}^{-/}$bound $\mathrm{e}^{-}$). The electron density is $\rho_{\mathrm{e}}=3.373 \times 10^{23} \mathrm{~cm}^{-3}$.

\begin{tabular}{llll|llll}
\hline $\begin{array}{l}\mathrm{X} \\
\left(\mathrm{nm}^{-1}\right)\end{array}$ & $F_{\text {coh }}$ & s.d. & $F_{\text {inc }}$ & $\begin{array}{l}\mathrm{x} \\
\left(\mathrm{nm}^{-1}\right)\end{array}$ & $F_{\text {coh }}$ & s.d. & $F_{\text {inc }}$ \\
\hline \hline $3.71 \mathrm{e}-001$ & 1.45 & 0.16 & 0.033 & $2.29 \mathrm{e}+000$ & 1.39 & 0.09 & 0.544 \\
$3.94 \mathrm{e}-001$ & 1.55 & 0.17 & 0.037 & $2.43 \mathrm{e}+000$ & 1.28 & 0.09 & 0.571 \\
$4.19 \mathrm{e}-001$ & 1.63 & 0.18 & 0.042 & $2.59 \mathrm{e}+000$ & 1.21 & 0.08 & 0.596 \\
$4.45 \mathrm{e}-001$ & 1.72 & 0.19 & 0.047 & $2.75 \mathrm{e}+000$ & 1.06 & 0.09 & 0.621 \\
$4.73 \mathrm{e}-001$ & 1.83 & 0.20 & 0.053 & $2.92 \mathrm{e}+000$ & 0.95 & 0.09 & 0.644 \\
$5.02 \mathrm{e}-001$ & 1.96 & 0.22 & 0.059 & $3.10 \mathrm{e}+000$ & 0.93 & 0.09 & 0.665 \\
$5.34 \mathrm{e}-001$ & 2.05 & 0.23 & 0.066 & $3.30 \mathrm{e}+000$ & 1.01 & 0.10 & 0.684 \\
$5.67 \mathrm{e}-001$ & 2.07 & 0.23 & 0.074 & $3.50 \mathrm{e}+000$ & 0.97 & 0.10 & 0.703 \\
$6.03 \mathrm{e}-001$ & 2.01 & 0.22 & 0.083 & $3.72 \mathrm{e}+000$ & 0.95 & 0.10 & 0.720 \\
$6.40 \mathrm{e}-001$ & 2.02 & 0.23 & 0.093 & $3.95 \mathrm{e}+000$ & 0.88 & 0.10 & 0.735 \\
$6.80 \mathrm{e}-001$ & 2.03 & 0.20 & 0.103 & $4.20 \mathrm{e}+000$ & 0.97 & 0.10 & 0.748 \\
$7.23 \mathrm{e}-001$ & 1.98 & 0.20 & 0.115 & $4.46 \mathrm{e}+000$ & 1.06 & 0.10 & 0.760 \\
$7.68 \mathrm{e}-001$ & 2.04 & 0.20 & 0.128 & $4.74 \mathrm{e}+000$ & 0.93 & 0.10 & 0.772 \\
$8.16 \mathrm{e}-001$ & 2.18 & 0.22 & 0.142 & $5.04 \mathrm{e}+000$ & 0.82 & 0.10 & 0.783 \\
$8.67 \mathrm{e}-001$ & 2.38 & 0.24 & 0.157 & $5.36 \mathrm{e}+000$ & 0.77 & 0.10 & 0.793 \\
$9.22 \mathrm{e}-001$ & 2.53 & 0.25 & 0.173 & $5.69 \mathrm{e}+000$ & 0.67 & 0.10 & 0.804 \\
$9.79 \mathrm{e}-001$ & 2.77 & 0.27 & 0.191 & $6.05 \mathrm{e}+000$ & 0.61 & 0.10 & 0.814 \\
$1.04 \mathrm{e}+000$ & 2.99 & 0.29 & 0.210 & $6.43 \mathrm{e}+000$ & 0.62 & 0.11 & 0.824 \\
$1.11 \mathrm{e}+000$ & 3.15 & 0.24 & 0.230 & $6.83 \mathrm{e}+000$ & 0.75 & 0.10 & 0.835 \\
$1.17 \mathrm{e}+000$ & 2.95 & 0.22 & 0.251 & $7.26 \mathrm{e}+000$ & 0.74 & 0.10 & 0.846 \\
$1.25 \mathrm{e}+000$ & 2.51 & 0.19 & 0.274 & $7.71 \mathrm{e}+000$ & 0.75 & 0.11 & 0.857 \\
$1.33 \mathrm{e}+000$ & 2.13 & 0.17 & 0.298 & $8.19 \mathrm{e}+000$ & 0.73 & 0.11 & 0.869 \\
$1.41 \mathrm{e}+000$ & 1.82 & 0.14 & 0.324 & $8.70 \mathrm{e}+000$ & 0.69 & 0.11 & 0.880 \\
$1.50 \mathrm{e}+000$ & 1.63 & 0.13 & 0.350 & $9.25 \mathrm{e}+000$ & 0.65 & 0.11 & 0.892 \\
$1.59 \mathrm{e}+000$ & 1.46 & 0.12 & 0.377 & $9.83 \mathrm{e}+000$ & 0.47 & 0.14 & 0.904 \\
$1.69 \mathrm{e}+000$ & 1.30 & 0.11 & 0.404 & $1.04 \mathrm{e}+001$ & 0.49 & 0.14 & 0.915 \\
$1.80 \mathrm{e}+000$ & 1.37 & 0.12 & 0.432 & & & & \\
$1.91 \mathrm{e}+000$ & 1.27 & 0.11 & 0.460 & & & & \\
$2.03 \mathrm{e}+000$ & 1.28 & 0.11 & 0.488 & & & & \\
$2.16 \mathrm{e}+000$ & 1.31 & 0.09 & 0.516 & & & & \\
\hline & & & & & & &
\end{tabular}


Table A.6: Measured form factor of nylon using the energy-dispersive technique. The units of $F_{\text {coh }}$ and its standard deviation (s.d.) are (free $\mathrm{e}^{-} /$bound $\left.\mathrm{e}^{-}\right)^{0.5}$ and the units of $F_{\text {inc }}$ are (free $\mathrm{e}^{-} /$bound $\mathrm{e}^{-}$). The electron density is $\rho_{\mathrm{e}}=3.753 \times 10^{23} \mathrm{~cm}^{-3}$.

\begin{tabular}{|c|c|c|c|c|c|c|c|}
\hline $\begin{array}{l}\mathrm{x} \\
\left(\mathrm{nm}^{-1}\right)\end{array}$ & $F_{\text {coh }}$ & s.d. & $F_{\text {inc }}$ & $\begin{array}{l}\mathrm{x} \\
\left(\mathrm{nm}^{-1}\right)\end{array}$ & $F_{\text {coh }}$ & s.d. & $F_{\text {inc }}$ \\
\hline $3.71 \mathrm{e}-001$ & 0.83 & $\overline{0.10}$ & 33 & $29 \mathrm{e}+000$ & $\overline{1.40}$ & $\overline{59}$ & 0.542 \\
\hline 3. & & & & & & & \\
\hline 170 & & 0.10 & 1 & 0 & 1.29 & & 0.5 \\
\hline 4.4. & & 0.10 & 0.046 & 0 & 1.23 & & 0.6 \\
\hline 4.7 & 0 & 0.10 & 0.052 & & 1.15 & 9 & 0.6 \\
\hline & 0.84 & 0.10 & 0.058 & & 1.12 & 0.09 & 0.666 \\
\hline 5. & 0.93 & 0.11 & 0.065 & & 1.14 & 0.10 & 0.685 \\
\hline 5. & 0.99 & 0.12 & 0.073 & & 1.10 & 0.10 & 0.704 \\
\hline 6.0 & 1.05 & 0.13 & 0.081 & & 1.04 & 0.10 & 0.722 \\
\hline 6.4 & 2 & 0.13 & 0.091 & & 1.07 & 0.10 & 0.739 \\
\hline 6.8 & 1.34 & 0.14 & 0.101 & 0 & 1.10 & 0.10 & 0.752 \\
\hline 7.2 & 1.34 & 0.14 & 0.113 & 00 & 1.04 & 0.10 & 0.765 \\
\hline 7.68 & 1.42 & 0.15 & 0.125 & 00 & 0.97 & 0.10 & 0.777 \\
\hline $8.1 \mathrm{c}$ & 55 & 0.16 & 0.139 & 5.04 & 0.92 & 0.10 & 0.789 \\
\hline 8.6 & 1.63 & 0.17 & 0.154 & 5.3 & 0.84 & 0.10 & 0.799 \\
\hline 9.2 & 1.71 & 0.17 & 0.171 & $5.69 e+000$ & 0.81 & 0.10 & 0.809 \\
\hline 9.7 & .93 & 0.20 & 0.188 & 6.05 & 0.60 & 0.10 & 0.820 \\
\hline 1.0 & .51 & 0.25 & 0.207 & 6.4 & 0.77 & 0.10 & 0.830 \\
\hline 1.1 & 3.51 & 0.26 & 0.227 & 6.8 & 0.77 & 0.10 & 0.840 \\
\hline 1.1 & 3.10 & 0.23 & 0.248 & 7.2 & 0.72 & 0.10 & 0.850 \\
\hline 1.2 & 2.77 & 0.21 & 0.271 & $7.71 \mathrm{e}$ & 0.81 & 0.11 & 0.860 \\
\hline 1.3 & 3.47 & 0.26 & 0.295 & $8.19 e+000$ & 0.69 & 0.11 & 0.870 \\
\hline 1.4 & 2.11 & 0.16 & 0.321 & $8.70 \mathrm{e}-$ & 0.66 & 0.11 & 0.881 \\
\hline $1.50 \mathrm{e}+000$ & 1.64 & 0.13 & 0.347 & $9.25 e+000$ & 0.67 & 0.11 & 0.891 \\
\hline $1.59 \mathrm{e}+000$ & 1.39 & 0.12 & 0.374 & $9.83 e+000$ & 0.58 & 0.12 & 0.902 \\
\hline $1.69 \mathrm{e}+000$ & 1.26 & 0.11 & 0.402 & $1.04 \mathrm{e}+001$ & 0.34 & 0.18 & 0.912 \\
\hline 1.8 & 1.24 & 0.11 & 430 & & & & \\
\hline 1. & 1. & 1 & 8 & & & & \\
\hline$e+000$ & 1.41 & 0 & 0 & & & & \\
\hline $2.16 \mathrm{e}+000$ & 1.50 & 0.09 & 0. & & & & \\
\hline
\end{tabular}




\section{APPENDIX B}

\section{ENERGY DisPERSIVE ALIGNMENT}

\section{PROCEDURE}

The alignment and characterization of the equipment for the energy dispersive form factor measurements in Chapters 5 and 6 is quite detailed. The procedure is given here. For the purposes of this procedure, the $\mathrm{z}$-dimension is defined to be in the direction defined by the line joining the x-ray tube and HPGe detector. The optical bench is aligned in this direction. The $\mathrm{x}$ and $\mathrm{y}$ dimensions are perpendicular to this line with the $\mathrm{y}$-dimension being horizontal and the $\mathrm{x}$-dimension vertical. The aperture names listed in this procedure are referenced to Figure 5.2.

\section{Equipment}

The equipment used in the energy dispersive measurement is shown in Figures B.1 and B.2. All equipment with the exception of the x-ray tube, ion chambers and HPGe detector are mounted on movable stages on the optical bench. A vernier scale provides precise positioning of each stage in the $\mathrm{z}$-direction while the stages can be adjusted in the $\mathrm{x}$ and $\mathrm{y}$ dimensions using precision screws. All elements are mounted on their stages with cylindrical rods. The rods can be rotated to adjust the angle of the equipment. All apertures must be mounted perpendicular to the z-direction. This is verified using the angle verification tool, which is shown in Figure B.2. 

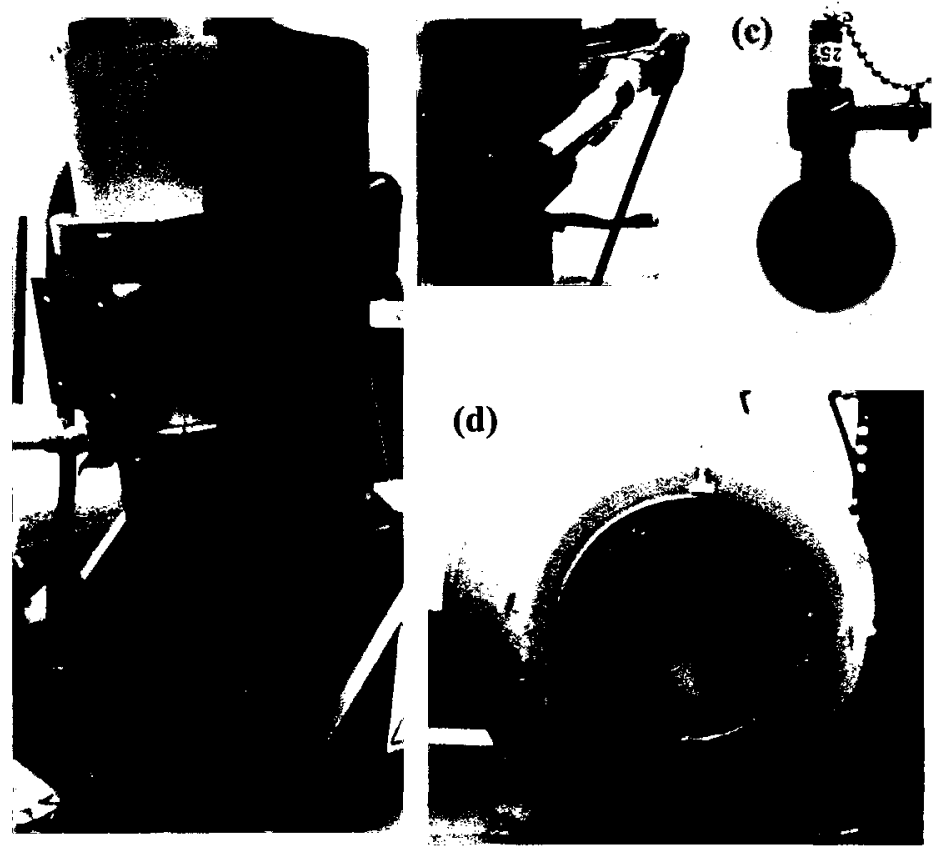

Figure B.1: Photograph of (a) X-ray tube, (b) tube monitor ion chamber, (c) pancake ion chamber and (d) front face of HPGe spectrometer used in energy-dispersive measurement.
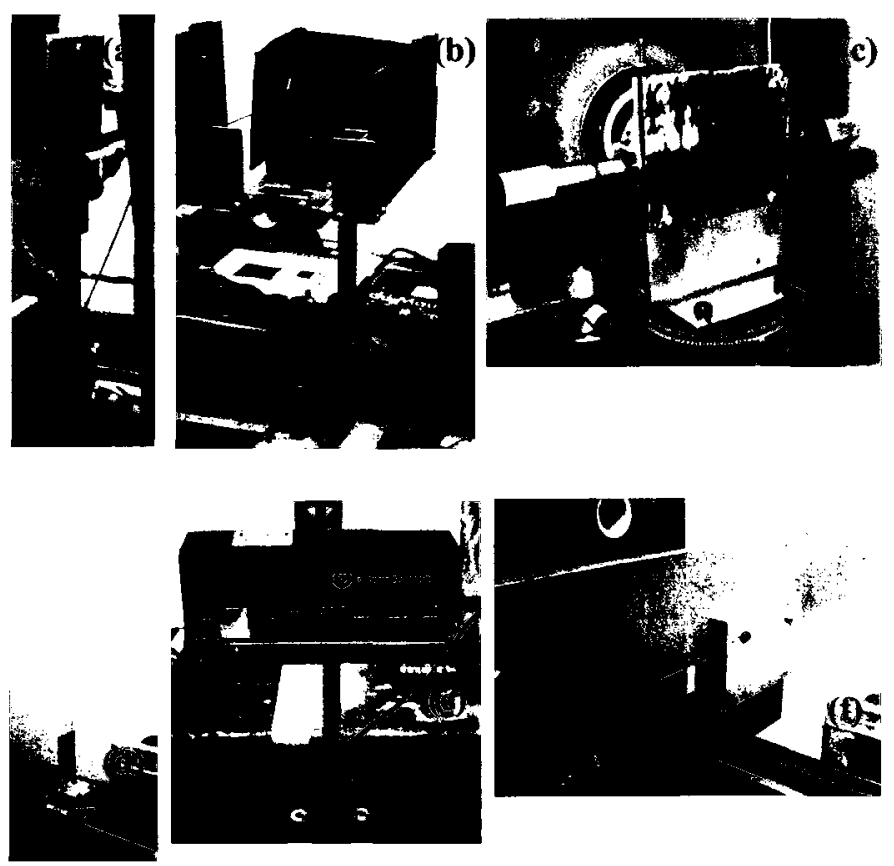

Figure B.2: Bench mounted equipment used in energy-dispersive form factor measurement: (a) Aperture C, (b) target station, (c) transmission aperture G, (d) bench marker, (e) Helium-Neon Laser, (f) Angle verification tool. 
The HPGe detector is mounted in a wheeled cabinet at one end of the optical bench, allowing coarse adjustment in the $\mathrm{y}$ and $\mathrm{z}$ dimensions but not in the $\mathrm{x}$-dimension. The $\mathrm{x}$-ray tube is mounted on a custom tube holder over the optical bench. Adjustment in all three directions is possible, though very difficult due to the weight of the $\mathrm{x}$-ray tube housing.

Throughout this experiment, a laser beam is often used to aid in alignment. The laser is mounted on an additional bench stage and must be aligned with the z-direction. This is accomplished by adjusting the alignment of the laser manually until the laser spot remains stationary on a bench marker as it slides along the bench.

\section{Location of x-ray tube focal spot}

The location where the $\mathrm{x}$-ray beam leaves the tube must be identified and marked. This is done by aligning the transmission opening of aperture $\mathrm{D}$ on the target station with aperture $\mathrm{C}$ using the laser beam. The laser beam then indicates the location on the tube housing that is visible through the two sets of apertures. An ion chamber is then located behind Aperture D and the signal strength through it is measured. The target station position is varied in the $\mathrm{x}$ and $\mathrm{y}$ dimensions while moving both apertures in synchrony using the laser beam. The signal strength is measured at each position with the ion chamber. The maximum strength corresponds to the location where the $\mathrm{x}$-ray beam leaves the tube. This should be marked for future reference.

\section{Alignment of x-ray tube and detector}

The laser beam is aligned such that it is pointing along the $\mathrm{z}$-axis at the centre of the HPGe detector. The target station is placed between the laser and the detector and the $\mathrm{x}$ and $\mathrm{y}$ position of the target station is varied until the transmission opening of aperture $\mathrm{D}$ is aligned with the laser beam. The laser is then re-aligned on the other side of the 
target station, pointing at the $\mathrm{x}$-ray tube and its location adjusted until the beam again points directly through the transmission opening of aperture $\mathrm{D}$. The laser beam now points along the line where the focal spot of the x-ray tube should be located. The x-ray tube should be moved to this position. The alignment of the $\mathrm{x}$-ray tube should be verified by finding the optimal position of the transmission opening of aperture D using the same ion chamber procedure as in the previous section. If the optimal location changes, the procedure outlined in this section must be repeated.

When this procedure is completed, the $\mathrm{x}$-ray tube, apertures $\mathrm{C}$ and $\mathrm{D}$ and the HPGe detector should all be aligned along the z-axis.

\section{Alignment of remaining transmission apertures}

The optimal position of the aperture $\mathrm{F}$ on the target station for a transmission measurement is determined by measuring the signal strength from the $\mathrm{x}$-ray tube with an ion chamber located behind the aperture. The largest signal strength at different aperture offsets defines the optimal position. The translatable aperture should be moved to this position.

Aperture $\mathrm{G}$ alignment is accomplished by first aligning the laser with the $\mathrm{z}$-axis, pointing at the centre of the HPGe detector. Aperture G is then placed on the optical bench and adjusted to line up with the laser beam. The micrometer spindles should be in the open position. At this point, a low current spectrum should be measurable by the HPGe detector if the laser is removed from the path. The $\mathrm{x}$ and $\mathrm{y}$ position of aperture $\mathrm{G}$ should be adjusted and a spectrum measured at each location. The optimal location is found where the largest count rate is measured. The spectrum measured at the optimal location should not show the presence of a $\mathrm{Pb} \mathrm{K}$-edge at $88 \mathrm{keV}$. If it does, the position or angular alignment is not correct and must be fixed.

The micrometer spindles should then be closed to limit the count rate at higher tube currents. Adjustments should be made until a reasonable dead time on the HPGe spec- 
trometer is measured at the desired tube voltage and current (2-3\% dead time using 121 $\mathrm{kV}, 2.0 \mathrm{~mA}$ is reasonable and achievable). After the jaws have been closed to the proper position, a low current $\mathrm{x}$-ray spectrum should be measured in order to characterize the effect of the tungsten spindles.

At this point, the transmission path should be completely aligned.

\section{Scatter aperture alignment}

Aperture $\mathrm{C}$ must be translated in the $\mathrm{y}$ direction to align with each scatter opening of aperture $\mathrm{D}$ on the target station. The optimal location of aperture $\mathrm{C}$ for each opening is measured by placing the ion chamber behind each of the openings in aperture $\mathrm{D}$ and measuring the $\mathrm{x}$-ray tube strength for different offsets of aperture $\mathrm{C}$. The optimal signal strength corresponds to the proper location of aperture $\mathrm{C}$ for each configuration.

Based on the known locations of the source, detector, and aperture D, the Matlab function MakeHolder_offset will calculate the proper vernier location to use for the target holder (plane E) and aperture F. The calculated value should be verified by placing a thin target in the target holder at the optimal location and measuring the scatter spectrum at different offsets for aperture $\mathrm{F}$. The maximum number of counts measured for each configuration corresponds to the true optimal position of aperture $F$.

At this point, the required offsets for apertures $\mathrm{C}$ and $\mathrm{F}$, as well as the proper offset for the target position have been determined for each scattering configuration. 


\section{APPENDIX C}

\section{SOURCE CODE DiRECTORY}

A complete listing of source code created during the writing of this thesis has been archived in the laboratory on compact disc. The files and their dependencies are listed here.

\section{C.1 Top-Level Programs}

The following top-level programs were written in Matlab (The Mathworks, Natick, MA). Their purpose and dependencies are listed for each program. Functions listed in italics are $\mathrm{C}++$ programs and their descriptions can be found in Section C.2. All of these programs were written by me except for ImageDisp which was written by Ossama Mousa. I modified this program to extract the diffraction signals from measured scatter patterns.

\section{simmonoFFCalc}

This program simulates the scatter pattern that would be generated by an object using a given $\mathrm{x}$-ray spectrum in an angle-dispersive configuration, then uses these data to extract the form factor using an effective energy approximation. 


\begin{tabular}{ll}
\hline Dependency & Description \\
\hline \hline SimScatter & Generate the simulated scatter pattern \\
$\Longrightarrow$ FFSim & See Section C.2 \\
AvEnergy & Compute the average energy \\
$\Longrightarrow$ Checkarg & Check validity of function arguments \\
KleinNishina & Compute $F_{K N}$ \\
$\Longrightarrow$ Checkarg & Check validity of function arguments \\
Checkarg & Check validity of function arguments \\
ReadSpectrum & Load a spectrum file \\
$\Longrightarrow$ BinSpectrum & Re-bin a given spectrum \\
$\Longrightarrow \Longrightarrow$ Checkarg & Check validity of function arguments \\
$\Longrightarrow$ SpectrumUtil & See Section C.2 \\
$\Longrightarrow$ Checkarg & Check validity of function arguments \\
\hline
\end{tabular}

\section{simsubFFCalc}

This program simulates the scatter pattern that would be generated by an object using a given $\mathrm{x}$-ray spectrum in an angle-dispersive configuration, then uses these data to extract the form factor using a sub-matrix calculation.

\begin{tabular}{ll}
\hline Dependency & Description \\
\hline \hline SimScatter & Generate the simulated scatter pattern \\
$\Longrightarrow$ FFSim & See Section C.2 \\
validsubmatrix & Determine the number of valid sub-matrices \\
$\Longrightarrow$ smooth & Rectangular smoothing \\
$\Longrightarrow \Longrightarrow$ Checkarg & Check validity of function arguments \\
$\Longrightarrow$ Checkarg & Check validity of function arguments \\
submatrix & Process sub-matrices \\
$\Longrightarrow$ ffsubcalc & Process individual sub-matrix \\
$\Longrightarrow$ Checkarg & Check validity of function arguments \\
Checkarg & Check validity of function arguments \\
\hline
\end{tabular}

\section{simregFFCalc}

This program simulates the scatter pattern that would be generated by an object using a given $\mathrm{x}$-ray spectrum in an angle-dispersive configuration, then uses these data to extract the form factor using a regularization calculation. 


\begin{tabular}{ll}
\hline Dependency & Description \\
\hline \hline SimScatter & Generate the simulated scatter pattern \\
$\Longrightarrow$ FFSim & See Section C. 2 \\
Checkarg & Check validity of function arguments \\
\hline
\end{tabular}

\section{compff_spec}

This program generates a series of triangular spectra of differing widths and skewness, simulates the scatter pattern for a given object with each spectrum, computes the form factor using a sub-matrix and regularization method, and determines the RMS difference between each calculated form factor and the exact form factor.

\begin{tabular}{ll}
\hline Dependency & Description \\
\hline \hline MakeSpectrum & Generate an artificial spectrum \\
$\Longrightarrow$ SpectrumUtil & See Section C.2 \\
simsubFFCalc & See Section C.1 \\
simregFFCalc & See Section C.1 \\
rmsdiff & Compute Root-Mean-Square difference \\
Checkarg & Check validity of function arguments \\
\hline
\end{tabular}

\section{compff_ff}

This program generates a series of artificial form factors of differing widths and peak locations, simulates the scatter pattern for a given spectrum with each form factor, computes the form factor using a sub-matrix and regularization method and determines the RMS difference between each calculated form factor and the exact form factor.

\begin{tabular}{ll}
\hline Dependency & Description \\
\hline \hline makeff & Generate an artificial form factor \\
$\Longrightarrow$ writefile & Write a text file \\
$\Longrightarrow$ checkarg & Check validity of function arguments \\
simsubFFCalc & See Section C.1 \\
simregFFCalc & See Section C.1 \\
rmsdiff & Compute Root-Mean-Square difference \\
Checkarg & Check validity of function arguments \\
\hline
\end{tabular}




\section{imagedisp}

This program is a general purpose image manipulation program originally written by Ossama Moussa. I added additional functionality to the program, primarily extending the program to extract circular diffraction pattern information from each image. In the interests of brevity, only the diffraction pattern extraction routines are listed here, although the entire program is available on the archived compact disc.

\begin{tabular}{ll}
\hline Dependency & Description \\
\hline \hline diffract & Collect scatter information \\
$\Longrightarrow$ circle & Draw circles on the image \\
$\Longrightarrow$ diff_extract & Extract scatter pattern \\
$\Longrightarrow \Longrightarrow$ excludeROIs & Exclude certain regions \\
$\Longrightarrow \Longrightarrow \Longrightarrow$ Checkarg & Check validity of function arguments \\
\hline
\end{tabular}

\section{sub_diffbg}

This program calculates the mean, background subtracted scatter signal from a series of scatter exposures as extracted from the imagedisp program.

\begin{tabular}{ll}
\hline Dependency & Description \\
\hline \hline writefile & Write a text file \\
Checkarg & Check validity of function arguments \\
\hline
\end{tabular}

\section{expmonoFFCalc}

This program reads the experimental scatter signal generated by the sub_diffbg function in an angle-dispersive configuration and extracts the form factor using an effective energy approximation. 


\begin{tabular}{ll}
\hline Dependency & Description \\
\hline \hline ExpScatter & Read the measured scatter pattern \\
$\Longrightarrow$ FFSim & See Section C.2 \\
AvEnergy & Compute the average energy \\
$\Longrightarrow$ Checkarg & Check validity of function arguments \\
KleinNishina & Compute $F_{\mathrm{KN}}$ \\
$\Longrightarrow$ Checkarg & Check validity of function arguments \\
Checkarg & Check validity of function arguments \\
ReadSpectrum & Load a spectrum file \\
$\Longrightarrow$ BinSpectrum & Re-bin a spectrum \\
$\Longrightarrow \Longrightarrow$ Checkarg & Check validity of function arguments \\
$\Longrightarrow$ SpectrumUtil & See Section C.2 \\
$\Longrightarrow$ Checkarg & Check validity of function arguments \\
\hline
\end{tabular}

\section{expsubFFCalc}

This program reads the experimental scatter signal generated by the sub_diffbg function in an angle-dispersive configuration and extracts the form factor using the sub-matrix calculation.

\begin{tabular}{ll}
\hline Dependency & Description \\
\hline \hline ExpScatter & Read the measured scatter pattern \\
$\Longrightarrow$ FFSim & See Section C.2 \\
validsubmatrix & Determine the number of valid sub-matrices \\
$\Longrightarrow$ smooth & Rectangular smoothing \\
$\Longrightarrow$ Checkarg & Check validity of function arguments \\
submatrix & Process all sub-matrices \\
$\Longrightarrow$ ffsubcalc & Process individual sub-matrix \\
$\Longrightarrow$ Checkarg & Check validity of function arguments \\
Checkarg & Check validity of function arguments \\
\hline
\end{tabular}

\section{expregFFCalc}

This program reads the experimental scatter signal generated by the sub_diffbg function in an angle-dispersive configuration and extracts the electronic form factor using the regularization calculation.

\begin{tabular}{ll}
\hline Dependency & Description \\
\hline \hline ExpScatter & Read the measured scatter pattern \\
$\Longrightarrow$ FFSim & See Section C.2 \\
Checkarg & Check validity of function arguments \\
\hline
\end{tabular}




\section{bkendispFFCalc4}

This program computes the coherent scattering form factor from energy-dispersive measurements of a given target for a series of spectra measured with different configurations. Results are computed for each individual configuration as well as a weighted result from all configurations. Quantitative uncertainty estimates are also computed.

\begin{tabular}{ll}
\hline Dependency & Description \\
\hline \hline ValidSourceArray & Check validity of source structures \\
$\Longrightarrow$ ValidSourceRectangle & Check for rectangular source \\
$\Longrightarrow \Longrightarrow$ checkarg & Check validity of function arguments \\
$\Longrightarrow$ ValidSourceCircle & Check for circular source \\
$\Longrightarrow \Longrightarrow$ Checkarg & Check validity of function arguments \\
$\Longrightarrow$ ValidSourceDefault & Check for default (circular) source \\
$\Longrightarrow \Longrightarrow$ Checkarg & Check validity of function arguments \\
$\Longrightarrow$ Checkarg & Check validity of function arguments \\
ValidDetectorArray & Check validity of detector structures \\
$\Longrightarrow$ ValidDetectorRectangle & Check for rectangular detector \\
$\Longrightarrow \Longrightarrow$ Checkarg & Check validity of function arguments \\
$\Longrightarrow$ ValidDetectorCircle & Check for circular detector \\
$\Longrightarrow \Longrightarrow$ Checkarg & Check validity of function arguments \\
$\Longrightarrow$ ValidDetectorDefault & Checkt for default detector (circular) \\
$\Longrightarrow \Longrightarrow$ Checkarg & Check validity of function arguments \\
$\Longrightarrow$ Checkarg & Check validity of function arguments \\
ValidApertureArray & Check validity of aperture structures \\
$\Longrightarrow$ ValidApertureRectangle & Check for rectangular aperture \\
$\Longrightarrow \Longrightarrow$ Checkarg & Check validity of function arguments \\
$\Longrightarrow$ ValidApertureCircle & Check for circular aperture \\
$\Longrightarrow \Longrightarrow$ Checkarg & Check validity of function arguments \\
$\Longrightarrow$ ValidApertureDefault & Check for default aperture (circular) \\
$\Longrightarrow \Longrightarrow$ Checkarg & Check validity of function arguments \\
$\Longrightarrow$ Checkarg & Check validity of function arguments \\
ValidObjectArray & Check validity of object structures \\
$\Longrightarrow$ ValidObject & Check validity of object \\
$\Longrightarrow \Longrightarrow$ Checkarg & Check validity of function arguments \\
$\Longrightarrow$ Checkarg & Check validity of function arguments \\
ValidTransap & Check validity of transmission aperture \\
$\Longrightarrow$ ValidTransap_simple & Check for simple aperture \\
$\Longrightarrow$ ValidTransap_pen & Check validity of function arguments \\
$\Longrightarrow$ Checkarg & Check for penetration aperture \\
$\Longrightarrow$ ValidTransap_window & Check validity of function arguments \\
& Check for energy window aperture \\
& \\
$\Longrightarrow$ & \\
$\Longrightarrow$ &
\end{tabular}




\begin{tabular}{|c|c|}
\hline$\Longrightarrow \Longrightarrow$ Checkarg & Check validity of function arguments \\
\hline ValidSpectra2 & Check validity of spectra \\
\hline$\Longrightarrow$ Checkarg & Check validity of function arguments \\
\hline bkCalcTargetParameters_mc & Calculate geometric parameters \\
\hline$\Longrightarrow$ line_intersect & Find intersection of two lines \\
\hline$\Longrightarrow$ rand_rect_norm & Create normal random numbers in rectangle \\
\hline$\Longrightarrow$ rand_circle & Create uniform random numbers in circle \\
\hline$\Longrightarrow \Longrightarrow$ rand_ring & Create uniform random numbers in a ring \\
\hline$\Longrightarrow$ rand_rect & Create uniform random number in rectangle \\
\hline ProcessSpectra4 & Process measured spectra \\
\hline$\Longrightarrow$ specratio & See Section C. 2 \\
\hline$\Longrightarrow$ BinSpectrum & Re-bin measured spectra \\
\hline$\Longrightarrow \Longrightarrow$ Checkarg & Check validity of function arguments \\
\hline$\Longrightarrow$ ArrayMatrixOperation & Perform matrix operation on many matrices \\
\hline$\Longrightarrow \Longrightarrow$ Checkarg & Check validity of function arguments \\
\hline$\Longrightarrow$ ArrayMatrixMultiply & matrix multiply many matrices \\
\hline$\Longrightarrow \Longrightarrow$ Checkarg & Check validity of function arguments \\
\hline$\Longrightarrow$ XToEnergy & Convert $x, \theta$ to energy \\
\hline$\Longrightarrow \Longrightarrow$ expand & Expand array to match another \\
\hline$\Longrightarrow \Longrightarrow$ Checkarg & Check validity of function arguments \\
\hline$\Longrightarrow$ Checkarg & Check validity of function arguments \\
\hline IAMdata & Read IAM data from file to extract $F_{\text {inc }}$ \\
\hline EnergyToX & Convert energy, $\theta$ to $x$ \\
\hline$\Longrightarrow$ expand & Expand array to match another \\
\hline$\Longrightarrow \Longrightarrow$ Checkarg & Check validity of function arguments \\
\hline FKN & Compute $F_{\mathrm{KN}}$ \\
\hline$\Longrightarrow$ expand & Expand array to match another \\
\hline$\Longrightarrow \Longrightarrow$ Checkarg & Check validity of function arguments \\
\hline FKNgradient & Compute gradient of $F_{\mathrm{KN}}$ \\
\hline$\Longrightarrow$ expand & Expand array to match another \\
\hline$\Longrightarrow \Longrightarrow$ Checkarg & Check validity of function arguments \\
\hline ArrayMatrixOperation & Perform matrix operation on many matrices \\
\hline$\Longrightarrow$ Checkarg & Check validity of function arguments \\
\hline ArrayMatrixMultiply & matrix multiply many matrices \\
\hline$\Longrightarrow$ Checkarg & Check validity of function arguments \\
\hline WeightedMean & Compute weighted mean of values \\
\hline$\Longrightarrow$ Checkarg & Check validity of function arguments \\
\hline checkarg & Check validity of function arguments \\
\hline
\end{tabular}

\section{CalcCovmat_mc}

This program computes the covariance matrix for the geometric parameters in the energydispersive form factor calculation for a given configuration. Each experimental parameter 
is randomly varied and the results used to calculate the covariance matrix. The covariance matrices are used in the energy-dispersive form factor calculation.

\begin{tabular}{ll}
\hline Dependency & Description \\
\hline \hline bkCalcTargetParameters_mc & Calculate geometric parameters \\
$\Longrightarrow$ line_intersect & Compute intersection of two lines \\
$\Longrightarrow$ rand_rect_norm & Create normal random numbers in rectangle \\
$\Longrightarrow$ rand_circle & Create uniform random numbers in circle \\
$\Longrightarrow \Longrightarrow$ rand_ring & Create uniform random numbers in ring \\
$\Longrightarrow$ rand_rect & Create uniform random numbers in rectangle \\
\hline
\end{tabular}

\section{MakeHolder_offset}

This program computes the proper locations for the adjustable components of the target station based on the geometric model of the experiment for the energy-dispersive form factor calculation.

\begin{tabular}{ll}
\hline Dependency & Description \\
\hline \hline CalcOffsets & Calculate the required offsets \\
\hline
\end{tabular}

\section{bkfluencefactor2}

This program computes the measured fluence factors for the given geometric model of the aperture configurations and ion chamber measurements. The ratio of signals in the scatter configuration compared to the transmission configuration is measured and compared to the expected value, based on a Monte Carlo simulation of the experiment. The results are used in the energy-dispersive form factor calculation.

\begin{tabular}{ll}
\hline Dependency & Description \\
\hline \hline bkICvolume_mc & Determine active volume of an ion chamber \\
$\Longrightarrow$ rand_rect_norm & Create normal random numbers in rectangle \\
$\Longrightarrow$ rand_circle & Create uniform random numbers in circle \\
$\Longrightarrow \Longrightarrow$ rand_ring & Create uniform random numbers in ring \\
\hline
\end{tabular}




\section{bkFitAttenSpectra2}

This program computes the effective area of the removable transmission aperture $(\mathrm{G})$ as explained in Section 5.1.1. It does this by taking the ratio, bin by bin, of spectra measured with the micrometer jaws open and with the jaws closed. It is also necessary to know the size of the aperture when the jaws are open. A straight line fit in a number of configurable energy windows is performed to be used in the energy-dispersive form factor calculation.

\begin{tabular}{ll}
\hline Dependency & Description \\
\hline \hline ReadSpectrum & Load a spectrum file \\
SpectrumUtil & See section C.2 \\
$\Longrightarrow$ BinSpectrum & Re-bin a spectrum \\
$\Longrightarrow \Longrightarrow$ Checkarg & Check validity of function arguments \\
Checkarg & Check validity of function arguments \\
\hline
\end{tabular}

\section{C.2 C++ programs}

Several $\mathrm{C}++$ programs and libraries were written to manipulate measured spectra throughout this work. Programs that were created are listed below. The libraries that these programs require can be found in the $\mathrm{C}++$ Libraries Section.

\section{SpectrumUtil}

This program is a general purpose spectrum manipulation utility. It is command line driven and allows spectra to be read, written or created in a variety of file formats. It also performs detector correction and background subtraction among other functions.

\begin{tabular}{ll}
\hline Dependency & Description \\
\hline \hline Objspectra & Manipulate spectra. \\
Objerror & Handle errors. \\
Objdataseries & Manipulate series of data. \\
Objfilter & Manipulate attenuation filters. \\
Objrandom & Create random numbers. \\
Objspline & Generate splines. \\
Objuserinterface & Interface between user and program. \\
\hline
\end{tabular}




\section{FFSim}

This program can either simulate the angle-dispersive scattering from a given object and $\mathrm{x}$-ray spectrum or read experimental scattering information from an external file. It then formulates the appropriate matrices and sub-matrices for calculating the form factor from the scattering information.

\begin{tabular}{ll}
\hline Dependency & Description \\
\hline \hline Objspectra & Manipulate spectra. \\
Objerror & Handle errors. \\
Objdataseries & Manipulate series of data. \\
Objfilter & Manipulate attenuation filters. \\
Objrandom & Create random numbers. \\
Objspline & Generate splines. \\
Objuserinterface & Interface between user and program. \\
\hline
\end{tabular}

\section{specratio_nc2}

This program reads the spectrum files required to compute the background-corrected ratio between a scatter spectrum and transmission spectrum for use in the energy-dispersive calculation. No detector correction is applied in this function. A similar program, specratio2, exists that performs the same function while applying the detector correction routine.

\begin{tabular}{ll}
\hline Dependency & Description \\
\hline \hline Objspectra & Manipulate spectra. \\
Objerror & Handle errors. \\
Objspline & Generate splines. \\
Objdataseries & Manipulate series of data. \\
\hline
\end{tabular}

\section{C.3 C++ Libraries}

The following libraries were written to aid in analyzing and manipulating spectra. All of these libraries were written by me with the exception of objRandom which was written by Agner Fog and distributed under the Gnu General Purpose License (GPL). 


\section{Objspectra}

This library has tools for manipulating spectra in various ways. It is composed of the following classes and routines:

\begin{tabular}{ll}
\hline File & Description \\
\hline \hline spectralbase & Base class for spectrum manipulation. \\
spectralpdf & Create custom made spectra. Inherits from spectralbase. \\
mcaspectrum & $\begin{array}{l}\text { Manipulate spectra from a Multichannel analyzer. } \\
\text { Inherits from spectralbase. }\end{array}$ \\
aptspectrum & Manipulate APT spectrum files. Inherits from mcaspectrum. \\
delspectrum & Manipulate DE1 spectrum files. Inherits from mcaspectrum. \\
spcspectrum & Manipulate SPC spectrum files. Inherits from mcaspectrum. \\
detector & Contains detector properties. \\
miscfunc & Miscellaneous spectrum functions. \\
\hline
\end{tabular}

\section{Objdataseries}

This library allows manipulation of series of data in an easy, consistent manner.

\begin{tabular}{ll}
\hline File & Description \\
\hline \hline datapoints & Stores and accesses a number of data elements. \\
dataseries & Stores and accesses a series of $x, y$ data. \\
datafunction & Stores function data. Inherits from dataseries. \\
\hline
\end{tabular}

\section{Objfilter}

This library creates spectral filters, based on material attenuation coefficients, that can be applied to spectrum objects.

\begin{tabular}{ll}
\hline File & Description \\
\hline \hline filter & $\begin{array}{l}\text { Create and manipulate spectral filters. Inherits from datafunction } \\
\text { (see Objdataseries). }\end{array}$ \\
\hline
\end{tabular}

\section{Objspline}

This library creates and manipulates splines for interpolation. Linear, quadratic and cubic splines are available. 


\begin{tabular}{ll}
\hline File & Description \\
\hline \hline spline & Create splines and compute interpolated values. \\
\hline
\end{tabular}

\section{Objrandom}

This library creates random numbers. It was written by Agner Fog and distributed under the Gnu General Public License.

\section{ObjUserInterface}

This library sets up a standard menu based user interface for creating more complicated programs.

\begin{tabular}{ll}
\hline File & Description \\
\hline \hline uinterface & Communicate options to and from the user. \\
optionlist & Store the options in the menu. \\
\hline
\end{tabular}

\section{Objerror}

This library handles errors from other classes.

\begin{tabular}{ll}
\hline File & Description \\
\hline \hline error & Track and handle errors. \\
\hline
\end{tabular}




\section{REFERENCES}

[1] H. E. Johns and J. R. Cunningham, The Physics of Radiology, 4th edition (Charles C Thomas, Springfield, Illinois, 1983).

[2] L. A. Lehmann, R. E. Alvarez, A. Macovski, W. R. Brody, N. J. Pelc, S. J. Riederer, and A. L. Hall, "Generalized image combinations in dual kvp digital radiography," Med. Phys. 8, 659-667 (1981).

[3] G. N. Hounsfield, "Computerized transverse axial scanning (tomography): Part 1. description of system," British Journal of Radiology 46, 1016-1022 (1973).

[4] R. J. Leclair and P. C. Johns, "X-ray forward-scatter imaging: Experimental validation of model," Med. Phys. 28, 210-219 (2001).

[5] E. F. Plechaty, D. E. Cullen, and R. J. Howerton, Tables and graphs of photon interaction cross-sections from 1.0-keV to $100-\mathrm{MeV}$ derived from the LLL evaluated nuclear data library, Technical Report UCRL-50400, volume 6, revision 1, Lawrence Livermore Laboratory, Livermore, California (1975).

[6] P. G. Lale, "The examination of internal tissues, using gamma-ray scatter with a possible extension to megavoltage radiography," Phys. Med. Biol. 4, 159-167 (1959).

[7] R. L. Clarke and G. Van Dyk, "A new method for measurement of bone mineral content using both transmitted and scattered beams of gamma-rays," Phys. Med. Biol. 18, 532-539 (1973).

[8] F. T. Farmer and M. P. Collins, "A new approach to the determination of anatomical cross-sections of the body by Compton scattering of gamma-rays," Phys. Med. Biol. 16, 577-586 (1971). 
[9] F. T. Farmer and M. P. Collins, "A further appraisal of the Compton scattering method for determining anatomical cross-sections of the body," Phys. Med. Biol. 19, 808-818 (1974).

[10] L. Brateman, A. M. Jacobs, and L. T. Fitzgerald, "Compton scatter axial tomography with x-rays: SCAT-CAT," Phys. Med. Biol. 29, 1353-1370 (1984).

[11] G. Harding, J. Kosanetzky, and U. Neitzel, "X-ray diffraction computed tomography," Med. Phys. 14, 515-525 (1987).

[12] G. Harding, M. Newton, and J. Kosanetzky, "Energy-dispersive x-ray diffraction tomography," Phys. Med. Biol. 35, 33-41 (1990).

[13] R. J. Leclair and P. C. Johns, "A semianalytic model to investigate the potential applications of x-ray scatter imaging," Med. Phys. 25, 1008-1020 (1998).

[14] R. J. Leclair and P. C. Johns, "Analysis of spectral blur effects in X-ray scatter imaging," Med. Phys. 26, 1811-1816 (1999).

[15] D. L. Batchelar, M. T. Davidson, W. Dabrowski, and I. A. Cunningham, "Bonecomposition imaging using coherent-scatter computed tomography: Assessing bone health beyond bone mineral density," Med. Phys. 33, 904-915 (2006).

[16] M. T. M. Davidson, D. L. Batchelar, S. Velupillai, J. D. Denstedt, and I. A. Cunningham, "Analysis of urinary stone components by $\mathrm{x}$-ray coherent scatter: characterizing composition beyond laboratory x-ray diffractometry," Phys. Med. Biol. 50, 3773-3786 (2005).

[17] J. H. Hubbell and I. Øverbø, "Relativistic atomic form factors and photon coherent scattering cross sections," J. Phys. Chem. Ref. Data 8, 69-105 (1979).

[18] P. C. Johns and M. J. Yaffe, "Coherent scatter in diagnostic radiology," Med. Phys. 10, 40-50 (1983). 
[19] A. H. Narten, X-ray diffraction data on liquid water in the temperature range $4^{\circ} \mathrm{C}$ $200^{\circ} \mathrm{C}$, Technical Report ORNL 4578, Oak Ridge National Laboratory, Oak Ridge, Tennessee (1970).

[20] J. Kosanetzky, B. Knoerr, G. Harding, and U. Neitzel, "X-ray diffraction measurements of some plastic materials and body tissues," Med. Phys. 14, 526-532 (1987).

[21] D. E. Peplow and K. Verghese, "Measured molecular coherent scattering form factors of animal tissues, plastics and human breast tissue," Phys. Med. Biol. 43, 2431-2452 (1998).

[22] G. Hura, J. M. Sorenson, R. M. Glaeser, and T. Head-Gordon, "A high-quality X-ray scattering experiment on liquid water at ambient conditions," J. Chem. Phys. 113, 9140-9148 (2000).

[23] P. C. Johns and M. P. Wismayer, "Measurement of coherent X-ray scatter form factors for amorphous materials using diffractometers," Phys. Med. Biol. 49, 5233-5250 (2004).

[24] M. Z. Hasan, Measurement of x-ray scattering form factors over a wide momentum transfer range, Master's thesis, Department of Physics, Carleton University, Ottawa, Ontario, Canada (2003).

[25] R. J. Leclair, M. M. Boileau, and Y. Wang, "A semianalytic model to extract differential linear scattering coefficients of breast tissue from energy dispersive $x$-ray diffraction measurements," Med. Phys. 33, 959-967 (2006).

[26] B. W. King and P. C. Johns, "Measurement of coherent scattering form factors using an image plate," Phys. Med. Biol. 53, 5977-5990 (2008).

[27] B. W. King and P. C. Johns, "Measurement of coherent scattering form factors using polychromatic x-ray sources and imaging detectors," Proceedings of the 52nd Annual 
Scientific Meeting, Canadian Organization of Medical Physicists, Saskatoon, SK, May 31-June 3, 2006, 245-247, (COMP, Kanata ON, CA) [Abstract: Med. Phys. 33, 2673 (2006)].

[28] B. W. King and P. C. Johns, "A sub-matrix method for extracting x-ray coherent scattering form factors from image plate data," Medical Imaging 2007: Physics of Medical Imaging, ed J. Hsieh and M. J. Flynn, Proc. SPIE 6510 (SPIE, Bellingham, WA USA) $65100 \mathrm{R} 1-12$.

[29] M. Z. Hasan and P. C. Johns, "Energy-dispersive technique to measure x-ray scattering form factors over a wide momentum transfer range," Poster \# MO-POS-145 at the 50th Annual Meeting of the Canadian Organization of Medical Phycicists, held in conjunction with the Canadian Association of Physicists, the Canadian Astronomical Society, and the Biophysical Society of Canada, Winnipeg, MB, June 13-16, 2004 [Abstract: Physics in Canada 60, 145 (2004)].

[30] B. W. King and P. C. Johns, "An energy dispersive technique for measuring x-ray coherent scattering form factors," Poster \# 264, Canadian Association of Radiation Oncology and Canadian Organization of Medical Physicists Joint Annual Meeting, Toronto, ON, October 9-13, 2007 [Abstract: Radiotherapy and Oncology 84 supplement 2, S75 (2007)].

[31] B. W. King and P. C. Johns, "An energy-dispersive technique to measure tissue x-ray coherent scattering form factors," Presentation WE-E-304A-03, American Association of Physicists in Medicine Annual Meeting, Anaheim, CA, July 26-30, 2009 [Abstract: Med. Phys. 36, 2786 (2009)].

[32] B. W. King and P. C. Johns, "Laboratory experience with a mechanically cooled high-purity germanium x-ray spectrometer," Poster \# 194, Canadian Association of Radiation Oncology and Canadian Organization of Medical Physicists Joint Annual 
Meeting, Toronto, ON, October 9-13, 2007 [Abstract: Radiotherapy and Oncology 84 supplement 2, S56 (2007)].

[33] C. M. Lederer and V. S. Shirley eds., Table of Isotopes, seventh edition (John Wiley \& Sons, New York, 1978).

[34] R. Birch, M. Marshall, and G. M. Ardran, Catalogue of Spectral Data for Diagnostic $X$-rays (The Hospital Physicists' Association, London, UK, 1979).

[35] D. W. L. Hukins, X-Ray Diffraction by Disordered and Ordered Systems (Pergamon Press, Oxford, UK, 1981).

[36] J. D. Jackson, Classical Electrodynamics, 3rd edition (John Wiley \& Sons, New York, 1999).

[37] J. A. Rowlands, "The physics of computed radiography," Phys. Med. Biol. 47, R123R166 (2002).

[38] M. Yaffe, K. W. Taylor, and H. E. Johns, "Spectroscopy of diagnostic x rays by a Compton-scatter method," Med. Phys. 3, 328-334 (1976).

[39] S. D. Conte and C. de Boor, Elementary numerical analysis: An algorithmic approach, International series in pure and applied mathematics, 2nd edition (McGrawHill, New York, NY, USA, 1972).

[40] G. H. Golub and C. F. Van Loan, Matrix Computations, 3rd edition (Johns Hopkins University Press, Baltimore, 1996).

[41] A. Neumaier, "Solving ill-conditioned and singular linear systems: A tutorial on regularization," SIAM Review 40, 636-666 (1998).

[42] P. C. Johns and J. S. C. Schulze, "Pinhole image characterization of x-ray tube focal spots using storage phosphor imaging technology," Poster \# 11, Canadian Organiza- 
tion of Medical Physicists Annual Meeting, Victoria, BC, July 21-24, 2009 [Abstract: Med. Phys. 36 (2009) (to appear)].

[43] S. Brandt, Data Analysis, 3rd edition (Springer, New York, NY, USA, 1998).

[44] F. A. Balogun, A. Brunetti, and R. Cesareo, "Volume of intersection of two cones," Radiat. Phys. Chem. 59, 23-30 (2000).

[45] D. R. Lide, ed., CRC Handbook of Chemistry and Physics, 89th edition (CRC Press, Boca Raton, FL, 2008).

[46] ICRP, Report of the Task Group on Reference Man, ICRP Publication 23, International Commission on Radiological Protection, Oxford, UK (1975).

[47] J. S. Olsen, B. Buras, and T. Jensen, "Influence of polarization of the incident beam on integrated intensities in x-ray energy-dispersive diffractometry," Acta Crystallographica A 34, 84-87 (1978).

[48] P. C. Johns and M. J. Yaffe, "Correction of pulse-height spectra for peak pileup effects using periodic and random pulse generators," Nucl. Instr. Meth. in Phys. Res. A 255, 559-581 (1987).

[49] J. H. Hubbell, "Summary of existing information on the incoherent scattering of photons, particularly on the validity of the use of the incoherent scattering function," Radiat. Phys. Chem. 50, 113-124 (1997).

[50] I. D. Jupp, P. T. Durrant, D. Ramsden, T. Carter, G. Dermody, I. B. Pleasants, and D. Burrows, "The non-invasive inspection of baggage using coherent x-ray scattering," IEEE Transactions on Nuclear Science 47, 1987-1994 (2000). 